\title{
Hořava-Lifshitz gravity and effective theory of the fractional quantum Hall effect
}

\author{
Chaolun $\mathbf{W} \mathbf{u}^{a}$ and Shao-Feng $\mathbf{W} \mathbf{u}^{b, a}$ \\ ${ }^{a}$ Kadanoff Center for Theoretical Physics and Enrico Fermi Institute, University of Chicago, \\ Chicago, Illinois 60637, U.S.A. \\ ${ }^{b}$ Department of Physics, Shanghai University, \\ Shanghai 200444, China \\ E-mail: chaolunwu@uchicago.edu, sfwu@shu.edu.cn
}

ABSTRACT: We show that Hořava-Lifshitz gravity theory can be employed as a covariant framework to build an effective field theory for the fractional quantum Hall effect that respects all the spacetime symmetries such as non-relativistic diffeomorphism invariance and anisotropic Weyl invariance as well as the gauge symmetry. The key to this formalism is a set of correspondence relations that maps all the field degrees of freedom in the HoravaLifshitz gravity theory to external background (source) fields among others in the effective action of the quantum Hall effect, according to their symmetry transformation properties. We originally derive the map as a holographic dictionary, but its form is independent of the existence of holographic duality. This paves the way for the application of HoravaLifshitz holography on fractional quantum Hall effect. Using the simplest holographic Chern-Simons model, we compute the low energy effective action at leading orders and show that it captures universal electromagnetic and geometric properties of quantum Hall states, including the Wen-Zee shift, Hall viscosity, angular momentum density and their relations. We identify the shift function in Hořava-Lifshitz gravity theory as minus of guiding center velocity and conjugate to guiding center momentum. This enables us to distinguish guiding center angular momentum density from the internal one, which is the sum of Landau orbit spin and intrinsic (topological) spin of the composite particles. Our effective action shows that Hall viscosity is minus half of the internal angular momentum density and proportional to Wen-Zee shift, and Hall bulk viscosity is half of the guiding center angular momentum density.

KEYWORDS: Gauge-gravity correspondence, Effective field theories, Holography and condensed matter physics (AdS/CMT)

ArXiv EPRINT: 1409.1178 


\section{Contents}

1 Introduction $\quad 1$

1.1 Aspects of the fractional quantum Hall effect 1

1.2 Symmetries and quantum Hall effective field theory 2

1.3 Holography: relativistic vs. non-relativistic symmetries 4

$\begin{array}{lll}1.4 & \text { Hořava-Lifshitz gravity theory } & 6\end{array}$

$\begin{array}{lll}1.5 & \text { Outline and notations } & 8\end{array}$

2 A non-relativistic field theory in $2+1$ dimensions $\quad 10$

$\begin{array}{lll}2.1 & \text { Microscopic action and local symmetries } & 10\end{array}$

$\begin{array}{lll}2.2 & \text { Comments on coupling to curved spacetime } & 14\end{array}$

2.3 Global time and relationship to Newton-Cartan geometry 14

3 Dual gravity theory in 3+1 dimensional bulk $\quad \mathbf{1 5}$

$\begin{array}{lll}3.1 \text { Bulk fields and their diffeomorphism } & 15\end{array}$

$\begin{array}{ll}3.2 & \text { Gauge conditions and residual gauge transformations } \\ \end{array}$

$\begin{array}{lll}3.3 & \text { Near-boundary behaviors of fields and transformations } & 18\end{array}$

$\begin{array}{lll}3.4 & \text { Holographic dictionary } & 19\end{array}$

3.5 Additional constraints 22

3.5.1 How not to interpret $v_{i} \quad 22$

3.5.2 How not to deal with $v_{i} \quad 23$

$\begin{array}{ll}\text { 3.5.3 Choice (I): a path integral constraint } & 25\end{array}$

3.5.4 Choice (II): a field constraint 26

4 Low energy Chern-Simons effective action of FQHE $\quad 27$

4.1 Holographic gauge Chern-Simons term 28

$\begin{array}{lll}4.2 & \text { Under the path integral constraint } & 30\end{array}$

4.3 Correlation functions of electromagnetic response 31

4.4 Under the field constraint 32

4.5 Holographic gravitational Chern-Simons term for $z>1$

5 Gravitational response and geometric properties of FQHE 35

5.1 Wen-Zee shift and Hall viscosity 35

5.2 Magnetization and momentum density 35

5.3 Vorticity and guiding center angular momentum density 36

$\begin{array}{ll}5.4 & \text { Internal angular momentum density } \\ 5.5 & 37\end{array}$

$\begin{array}{ll}\text { 5.5 Bulk and total Hall viscosities } & 39\end{array}$

$\begin{array}{lll}5.6 & \text { Relations to Hall conductivity } & 39\end{array}$

5.7 Interpretation of two types of angular momentum density 40

5.7.1 Classical charged particles in electromagnetic field 40

5.7.2 Quantum mechanics: two Hilbert spaces 41

5.8 Guiding center momentum density 43 
B Near-boundary vectorial structures and diffeomorphism

C Calculating the gravitational Chern-Simons term

C.1 Bulk spin connection 52

C.2 Pontryagin density in the bulk 53

$\begin{array}{lll}\text { C.3 Pontryagin current at the boundary } & 54\end{array}$

\section{Introduction}

\subsection{Aspects of the fractional quantum Hall effect}

The fractional quantum Hall effect (FQHE) [1] has been a fascinating subject that attracts physicists from many different areas of both experimental and theoretical physics over the last three decades. The crucial role played by interactions between electrons poses a huge challenge to theorists and prevents the problem from being solved exactly till these days. Despite this difficulty, many different theoretical approaches to the problem has been developed since Laughlin's ground-breaking work on the trial wave function [2]. These approaches employ many different concepts and theoretical tools from various fields of modern physics and even mathematics, to reveal diverse aspects of rich physics encoded in this simple phenomenon.

One class of these approaches is based on field theories [3-7], particularly the ChernSimons gauge theory in $2+1$ dimensions. However, such approaches have shortages and limitations. One of the problems is the difficulty to deal with the massless limit. In the massless limit, the cyclotron frequency goes to infinity and the inter-Landau level gap becomes much larger than the Coulomb energy gap, which only depends on the magnetic field. This corresponds to the projection to the lowest Landau level (LLL). In many field theoretical approaches, such as the composite boson [3] and composite fermion [4] theories, the inter-Landau level mixing causes the LLL projection to be unnatural and many quantities which should depend only on Coulomb energy scale are also sensitive to cyclotron frequency. The smoothness of the massless limit is a requirement for a field theory formalism to be an adequate description of the quantum Hall effect.

At the low energy (much lower compared to the Coulomb gap) effective theory level, Chern-Simons field theory is believed to encode all the universal properties of quantum Hall states [5]. However, in addition to the gauge degrees of freedom related to electromagnetic properties such as the quantized Hall conductivity [8-10], fractionally charged (anyonic) excitations $[2,11,12]$ and chiral edge states [13-15] that are well described by gauge ChernSimons theory [16, 17], the fractional quantum Hall states also possesses another sets of degrees of freedom, which is called the "geometric degrees of freedom" by Haldane [18]. 
This is another hallmark of the fractional quantum Hall effect that makes it fundamentally different from and more complicated than the integer quantum Hall effect [19]. The origin of these two sets of degree of freedom can be traced back to the structure of Hilbert space of charged particles in magnetic field $[20,21]$ and the underlying symmetry of $W_{\infty}$ algebra [22]. Early study related to the geometric degrees of freedom dated back to the magneto-roton theory of collective neutral excitations [23]. A new quantum number, the shift $\mathcal{S}$, was introduced in [24] as the coefficient of a new Chern-Simons-type term, the Wen-Zee term

$$
\frac{\mathcal{S}}{4 \pi} \int \omega \wedge \mathrm{d} A
$$

where $A$ is the $\mathrm{U}(1)$ gauge field and $\omega$ is the Abelian spin connection associated with the 2dimensional spatial manifold. The shift is topological in nature and its role to characterize gravitational response of fractional quantum Hall states is similar to that of the quantized Hall conductivity to the electromagnetic response. The topological phases of fractional quantum Hall states are determined by Hall conductivity and the shift together, not the former alone. Although the shift is originally introduced to describe quantum Hall states on curved manifolds with non-trivial topology, it also manifests itself in states on flat manifolds as a parity-odd dissipationless transport coefficient known as Hall viscosity [25-36], ${ }^{1}$ which in turn is related to the angular momentum density in gapped systems [30, 47-49]. Recently, Haldane proposed a geometric point of view [18] to renew the understanding of fractional quantum Hall dynamics, in which the Hall viscosity [50-52] and neutral collective excitations [53] are related to the fluctuations of guiding center metric defined in the 2-dimensional phase space of the non-commutative guiding center coordinates. A closely related topic is the study of the nematic degrees of freedom, whose dynamics is also governed by the Wen-Zee term. Inspired by early works of $[54,55],[56,57]$ propose field theoretical descriptions of the quantum Hall nematic transition in which an isotropic Laughlin liquid undergoes phase transition to a nematic state with the same filling factor but spontaneously breaks rotational symmetry, and identify the order parameter as unimodular spatial metric components.

\subsection{Symmetries and quantum Hall effective field theory}

In field theoretical approaches, symmetries play an important role in building phenomenological models as well as solving and subtracting physical information. Gauge invariance and Galilean symmetry, particularly the former one, have been well known to be crucial to the understanding of quantum Hall effects [58]. However, these are not the only symmetries that are relevant and can be utilized in the study. Non-relativistic systems possess a larger set of symmetries than the aforementioned two. In [59] the notion of non-relativistic diffeomorphism invariance is introduced, and further enlarged to its maximal in [60] for quantum Hall systems to accommodate arbitrary spacetime diffeomorphism, a general gyromagnetic

\footnotetext{
${ }^{1}$ A closely related situation is Hall viscosity in chiral superfluids [37-40] (in addition to [29, 30]). Hall viscosity in relativistic systems has also been studied in [41-44] and [45, 46]. The latter is related to quantum Hall effects in graphene. But since the spacetime symmetry is different in these cases, they are not considered here.
} 
factor and intrinsic spin. It is first used to construct an effective action for quantum Hall effects in [31] to explain the relation between Hall viscosity and the inhomogeneous magnetic field correction to the Hall conductivity. [61] introduces the Newton-Cartan geometry as a mathematical machinery that manifests the non-relativistic diffeomorphism invariance in a covariant way, and constructs an effective action that resolves the unnaturalness problem of LLL projection and encodes most of the universal properties of fractional quantum Hall states in a natural and unified way. The Newton-Cartan formalism is further used to study spectral density sum rules [62], conservation laws and Ward identities [60], hydrodynamics [63] of quantum Hall fluids and extended to include torsion [60, 64]. Recently, [65] offers an extensive formal discussion on algebraic aspects of Newton-Cartan geometry as a formalism of Galilean-invariant field theories. Non-relativistic diffeomorphism invariant effective actions can also be constructed without using Newton-Cartan formalism [66-69]. Some recent general discussions on non-relativistic spacetime symmetries can be found in [70-72], and similar ideas are applied to construct low energy effective action for non-relativistic gapped systems in [73].

The idea behind the approach of $[31,61]$ is that, a single gauge Chern-Simons term $\int A \wedge \mathrm{d} A$ alone as the leading order term of the low energy effective theory does not respect the full spacetime symmetries of the underlying microscopic field theory. The nonrelativistic diffeomorphism invariance, along with other constraints such as the naturalness of LLL projection, requires a series of additional terms and fixes the relative coefficients between them. This is how other universal properties of fractional quantum Hall states mentioned earlier are embedded into the Chern-Simons theory. Strictly speaking, the local non-relativistic diffeomorphism invariance is just a spurionic symmetry of the system. It does not induce a conservation law per Noether's theorem. It is not even uniquely determined because to read it off the underlying microscopic field theory has to be coupled to curved spacetime first and this coupling is not unique. Only a subgroup of the full diffeomorphism invariance that preserves a certain background (usually flat) is the true symmetry (the Galilean or Schrodinger algebra). However, the full diffeomorphism invariance is a powerful tool to constrain the form of the effective action and to subtract physical information such as Ward identities higher correlation functions and relations between transport coefficients at the low energy level.

Another point to note is that, given a specific form of non-relativistic diffeomorphism invariance (i.e. the coordinate transformation rules for all fields and parameters in the theory that keep the action and effective action invariant), there may exist more than one covariant formalisms of the geometry one can use as the machinery to build up the effective theory. Depending on the purpose, one may be more convenient than another or vice versa. The Newton-Cartan formalism introduced in [61], with its non-relativistic nature [74] and rich structure, becomes a popular one to describe quantum Hall effects. In this paper, however, we will employ a different covariant formalism - the Hořava-Lifshitz gravity theory, and show that it is an equally powerful framework for quantum Hall effective theory. It has one notable advantage over the Newton-Cartan formalism. It is closely associated to relativistic gravity theories (for example, through the khronon formalism [75, 76]), thus provides a natural connection to the holographic approach, which has been developed so far mostly within the frameworks of relativistic gravity and string theories. 
The idea of using Hořava-Lifshitz gravity theory to construct effective actions with non-relativistic diffeomorphism invariance (non-holographically) has already been introduced in [66] (followed by [77]), but their formalism is limited in several aspects. Their non-relativistic diffeomorphism is not the most general one for quantum Hall effect. Their construction relies on taking the non-relativistic limit $c \rightarrow \infty$ of some relativistic theory, which will usually not give the most general results as to directly consider the diffeomorphism itself, thus is over-constrained. Last but not least, in their metric ansatz they identify the $\mathrm{U}(1)$ field directly as the subleading order metric components in $1 / c$ expansion. We think the last part is the major limitation and we will introduce the $\mathrm{U}(1)$ field separately in our formalism, independent of the graviton sector, and will identify the shift function in the metric as something different in the context of quantum Hall effects.

\subsection{Holography: relativistic vs. non-relativistic symmetries}

Holography, or the gauge/gravity duality [78-80], has been a powerful tool to study strongly-correlated quantum systems observed or theoretically proposed in many different areas of modern physics. Its higher dimensional geometric approach gives dual descriptions of quantum field theories at strong coupling regime, which is complementary to the traditional perturbative methods. An early triumph of holography in high energy physics is the prediction of the lower bound of shear viscosity to entropy density ratio of strongly coupled quark-gluon plasma [81-84]. Holography has also been applied to many condensed matter systems and sheds new light on many age old problems, offering new perspectives and efficient computational methods (for an incomplete list of reviews of some most popular topics, we refer to [85-91]). A topic related to the current discussion is Lifshitz holography, the holographic dual to field theories with Lifshitz scaling, starting from [92]. It typically employs relativistic gravity coupled to various types of matter fields, among which the most popular version is introduced in [93]. Of particular interest are the recent work of [94, 95] where the boundary geometry of $z=2$ Lifshitz holography under certain conditions for the time-like vielbein is identified with Newton-Cartan geometry with or without torsion, and of $[96,97]$ where a systematic approach for constructing holographic dictionary for Lifshitz holography is presented. Instead of using relativistic gravity coupled to matters, we will use Hořava-Lifshitz gravity to realize the Lifshitz holography. It has Lifshitz geometry as a vacuum gravity solution without coupling to matters.

The fractional quantum Hall effect, a state of quantum fluids largely due to the strong correlations of electrons through Coulomb interaction in high magnetic field, is another natural playground for holography. Early researches based on either bottom-up phenomenological approaches or top-down string/brane settings turn out to be fruitful [98-111]. However, one of the limitations of most of these studies is that they focus primarily on electromagnetic properties of the quantum Hall phenomena, particularly the conductivity. Most of the geometric properties that also characterize quantum Hall states, such as the Wen-Zee shift and Hall viscosity, are overlooked, thus the holographic approach to quantum Hall effects so far is still incomplete. One purpose of this paper is to fill in this gap. The origin of this problem can be traced back to the spacetime symmetry of these holographic models. Since they are all built in within the framework of string theory or relativistic 
gravity theory, their spacetime symmetry is relativistic by nature. This does not match the non-relativistic diffeomorphism invariance of [59-61], which is more appropriate for the description of quantum Hall effects and is crucial to the encoding of geometric properties into the effective description. For example, as the $(2+1)$-dimensional gauge Chern-Simons term $A \wedge \mathrm{d} A$ can easily find its dual description in (3+1)-dimensional holographic model as the bulk Chern-Simons term $\mathrm{d} A \wedge \mathrm{d} A$, the Wen-Zee term $\omega \wedge \mathrm{d} A$ can not because the spin connection in relativistic (3+1)-dimensional bulk is naturally non-Abelian and a term like $\mathrm{d} \omega \wedge \mathrm{d} A$ is forbidden by symmetry. Our solution to this problem is to use a non-relativistic gravity theory - the Hořava-Lifshitz gravity - as the bulk description where a Wen-Zee term is not forbidden by symmetry at least at the boundary and thus can be induced by the holographic dictionary.

A guiding principle to construct a dual holographic model for a certain quantum field theory is the matching of all symmetries on both sides. This is especially important for the bottom-up approach where means of constraining the form of the action are limited. In classic examples of AdS/CFT correspondence [112], the full conformal and flavor symmetries of the field theories are completely realized by the background isometries of the dual gravity theories. This is necessary for the top-down approach, but for the bottom-up approach which we will follow here, especially for the applications to condensed matter physics, this requirement that the full global symmetries are realized by background isometries might be too strong and not necessary. A weaker condition that the holographic on-shell action encodes all the field theory symmetries is sufficient in practice. This means that the bulk background isometry does not have to incarnate the full symmetry algebra [113]. This weak requirement is particularly useful for building up bottom-up models for systems with non-relativistic symmetries to avoid certain complications such as excessive extra dimensions. The difference between the strong and weak requirements is that in the latter the holographic dictionary will be more complicated and play a more vivid role to realize the rest of the symmetry that is not realized by the isometry. In fact, according to the standard holographic dictionary,

$$
\mathcal{I}_{\text {grav }}[\bar{\phi}]=\mathcal{W}_{\mathrm{QFT}}[J], \quad \bar{\phi}=J,
$$

where $\mathcal{I}_{\text {grav }}$ is the on-shell action of the (weakly coupled or classical) gravity theory and $\mathcal{W}_{\mathrm{QFT}}$ the effective action ${ }^{2}$ of the dual (strongly-coupled) quantum field theory. $\bar{\phi}$ denotes a collection of normalizable mode coefficients of the bulk fields, which are usually called the boundary fields and identified as the source field $J$ to some operator $\hat{\mathcal{O}}$ in the quantum field theory. The form of $\mathcal{W}_{\mathrm{QFT}}[J]$ is highly constrained by the symmetries of the quantum field theory, then so is that of $\mathcal{I}_{\text {grav }}[\bar{\phi}]$ according to the dictionary. Thus the necessary and sufficient condition for the matching of symmetries is that the on-shell action $\mathcal{I}_{\text {grav }}[\bar{\phi}]$ respects symmetries of the field theory. This is a combined result of the bulk background isometry (particularly near the boundary) and the holographic dictionary, not only the former one alone. In the classic AdS/CFT correspondence [112] where the holographic

\footnotetext{
${ }^{2}$ Throughout this paper, by "effective action" $\mathcal{W}$, we simply mean the logarithm of the generating functional $\mathcal{Z}$ of the quantum field theory, as a functional of the source $J: \mathcal{W}[J]=-i \log \mathcal{Z}[J]$.
} 
dictionary takes a simple and trivial form (for example, the boundary gauge field maps exactly to the source of the conserved current of the same gauge group in the field theory), the requirement of fulfilling the symmetries falls upon completely on the background isometry. In non-relativistic holography, this is not always true, or at least certainly not as cheap as that for the relativistic. In early works of $[114,115],{ }^{3}$ for example, the spacetime symmetry group for unitary fermions, the Schrodinger group, is fully realized by the background isometry, but at the price of introducing two extra dimensions rather than one in relativistic cases. Furthermore, this type of models suffer problems such as zero modes and infinite Kaluza-Klein towers in the mass spectrum resulting from the discrete light-cone quantization (DLCQ). They can hardly be the holographic duals of generic non-relativistic field theories. A first step toward a more generic setup by getting rid of the second extra dimension and giving up the strong requirement on bulk isometry is made by [113], where an extra gauge field has to be introduced and the holographic dictionary becomes less trivial by mixing the metric and the gauge field. [117, 118] makes a further step by directly employing a non-relativistic bulk gravity theory - Hořava-Lifshitz gravity, and building the holographic dictionary by matching to the non-relativistic diffeomorphism invariance of [59]. In this paper, we will follow closely the philosophy of $[117,118]$ and extend their approach to the parity-violating case by building a holographic dictionary that matches the non-relativistic local spacetime and gauge symmetries of [60]. This offers a general platform for building more detailed holographic models dual to strongly-coupled spin-polarized charged particle systems in magnetic field, particularly the quantum Hall effects.

\subsection{Hořava-Lifshitz gravity theory}

In the above when we are talking about matching the symmetries of a field theory and its holographic dual, we mean the full local symmetries, including the "spurionic" diffeomorphism invariance and local gauge invariance, not just the global symmetries. A detailed explanation of this point and its connection with building non-relativistic holographic dictionary is presented in [118]. In non-relativistic systems, there is a preferred notion of time - the global time, which defines simultaneity and is a consequence of non-relativistic causality. The general coordinate transformations that preserve the global time foliation on the manifold involve an arbitrary time-dependent time reparametrization and an arbitrary time- and space-dependent spatial diffeomorphism:

$$
\delta t=-\xi^{t}(t), \quad \delta x^{i}=-\xi^{i}\left(t, x^{j}\right)
$$

which is usually called foliation preserving diffeomorphism (FPD). Here $i$ and $j$ run through all spatial directions. Non-relativistic diffeomorphism invariance is a statement that physics is invariant under these FPD. To build up the holographic dual, it is natural to seek a gravity theory with the same causal structure, notion of global time and diffeomorphism invariance. The candidate is Hořava-Lifshitz gravity theory [119, 120], a gravity theory that is constructed to be invariant under the above FPD. The gravity sector includes the lapse function $N$, shift function $N_{i}$ and spatial metric $g_{i j}$, which can be viewed as the

\footnotetext{
${ }^{3}$ For a review from algebraic point of view, we refer to [116].
} 
Arnowitt-Deser-Misner (ADM) decomposition of the full spacetime metric [121]:

$$
d s^{2}=-N^{2} d t^{2}+g_{i j}\left(d x^{i}+N^{i} d t\right)\left(d x^{j}+N^{j} d t\right) .
$$

Their transformation under FPD will be listed later when we talk about the bulk theory of the holography. Time derivative appears only in the extrinsic curvature

$$
K_{i j}=\frac{1}{2 N}\left(\partial_{t} g_{i j}-\nabla_{i} N_{j}-\nabla_{j} N_{i}\right)
$$

where $\nabla_{i}$ is the covariant derivative associated with the spatial metric $g_{i j}$. We define $K=g^{i j} K_{i j}$.

Hořava-Lifshitz gravity is originally proposed as a ultraviolet-complete quantum gravity theory to describe the real world. For this purpose, there are many phenomenological and cosmological issues under debates where there exists an extensive literature (for some early reviews and references, see $[122,123])$. But these are mostly irrelevant to us, since we are only interested in using the theory as a framework to build up effective theories or holographic duals for non-relativistic field theories. We do not need to assume projectability or detailed balance as introduced in [120]. We are mostly interested in the classical low energy limit (analog to the large $N_{c}$ limit of the relativistic AdS/CFT correspondence) of the theory where the lowest derivative terms dominate. The general form of the leading order graviton action up to two-derivative terms is [75, 124, 125]:

$$
S_{\text {grav }}=\frac{1}{16 \pi G_{N}} \int d t d^{d} \vec{x} \sqrt{g} N\left\{K_{i j} K^{i j}-(1+\tilde{\lambda}) K^{2}+\beta(R-2 \Lambda)+\alpha \frac{\left(\nabla_{i} N\right)\left(\nabla^{i} N\right)}{N^{2}}\right\} .
$$

where $R$ is the Ricci scalar associated with $g_{i j}$ and $\Lambda$ cosmological constant and spatial indices $i$ and $j$ are raised and lowered by $g^{i j}$ and $g_{i j}$. $G_{N}$ is Newton's constant, but as part of effective theory for condensed matter systems, it will be mapped to some parameters in the problem under consideration. When $\alpha=\tilde{\lambda}=0$ and $\beta=1$ the action goes back to Einstein-Hilbert form. This low energy form of Hořava-Lifshitz gravity is a limiting case $[126,127]$ of the Einstein-aether theory [128-130] with hypersurface orthogonal aether field. We will also need a $\mathrm{U}(1)$ gauge field $V_{\mu}=\left(V_{t}, V_{i}\right)$, whose low energy action up to two derivatives has the general form [131]

$$
S_{\text {gauge }}=\frac{1}{4 g_{e}^{2}} \int d t d^{d} \vec{x} \sqrt{g} N\left\{\frac{2}{N^{2}} g^{i j}\left(V_{t i}-N^{k} V_{k i}\right)\left(V_{t j}-N^{l} V_{l j}\right)+f\left[V_{1}, V_{2}\right]\right\},
$$

where $V_{\mu \nu}=\partial_{\mu} V_{\nu}-\partial_{\nu} V_{\mu}, g_{e}$ is the gauge coupling and $f$ is an arbitrary scalar functional of its arguments. For $d=3, V_{1}=g^{i j} \mathfrak{B}_{i} \mathfrak{B}_{j}, V_{2}=\nabla_{i} \mathfrak{B}_{j} \nabla^{i} \mathfrak{B}^{j}$ and $\mathfrak{B}_{i}=\frac{1}{2} \varepsilon_{i}{ }^{j k} V_{j k}$. For $d=2$, $V_{1}=\mathfrak{B}, V_{2}=\nabla_{i} \mathfrak{B} \nabla^{i} \mathfrak{B}$ and $\mathfrak{B}=\frac{1}{2} \varepsilon^{i j} V_{i j}$. Here $\varepsilon$ with indices is the Levi-Civita tensor associated with the metric $g_{i j}$ in specific dimensions. The form of a scalar action is also discussed in [131], but we will not need them explicitly in this paper. The Chern-Simons terms can be added in a similar way as in relativistic theories. We will discuss this in great details in late sections. In $2+1$ dimensions, a FPD invariant Wen-Zee term can be added as

$$
S_{\mathrm{WZ}}=\frac{\mathcal{S}}{4 \pi} \int \omega^{\prime} \wedge \mathrm{d} V
$$


where $\omega^{\prime}=\omega+\ldots$ is a covariant Abelian spin connection defined on the 2-dimensional spatial manifold. The $\omega^{\prime}$ is different from the Abelian spin connection $\omega$ in the classic WenZee term introduced in [24] by the "..." part, because $\omega$ is not a FPD covariant 1-form. The precise definition of $\omega^{\prime}$ can be found in (3.36) and (3.37).

In $[132,133]$ the Hořava-Lifshitz gravity described above are extended. A local $\mathrm{U}(1)$ symmetry called $\mathrm{U}(1)_{\Sigma}$ is introduced and the FPD invariance is upgraded to "nonrelativistic general covariance". Along with these is the introduction of some additional fields into the theory, such as the "Newton potential" $A$ and "Newton prepotential" $\nu$. They are related to the subleading terms in the metric in the non-relativistic limit where the speed of light $c \rightarrow \infty$. [117] discusses their roles in holographic model building. Since our theories have only FPD symmetry, not the non-relativistic general covariance, we will not introduce these fields in our model. This agrees with the spirit of [117].

Related to applications on holography, Hořava-Lifshitz gravity theory admits Lifshitz solution [117, 134], hyperscaling violating solutions [135] and asymptotic AdS or Lifshitz black hole solutions [136-138].

We have a few more simplifications compared to [117]. They introduce a field $P_{i}$, the $O\left(c^{2}\right)$ order of the shift function. This is related to the field $C_{i}$ introduced in [60] to source the energy flux when $\partial_{i} \xi^{t} \neq 0$. Since we are always staying in the global time coordinates, they can be consistently set to zero. [117] also introduces a second U(1) field called $b_{\mu}$, the $O\left(c^{2}\right)$ order of the gauge field in the parent relativistic theory. Its main function is to provide a chemical potential related to the mass $m$ and it enters the holographic model mainly through the combination $b_{t} / N$, which is a scalar. Thus we will not introduce this additional $\mathrm{U}(1)$ field, but instead just a scalar field $\phi$ functioning as their $b_{t} / N$. The meaning of $\phi$ will be discussed once its holographic map is built. Throughout this paper, we will not introduce the speed of light $c$ in our construction, nor the non-relativistic limit $c \rightarrow \infty$. In other words, we have already set $c=\infty$. The FPD of fields and form of the effective action are not obtained by taking non-relativistic limit of some relativistic parent theory, such as in [66] and part of [117]. This allows maximal generality in the construction.

\subsection{Outline and notations}

In this paper we show that Hořava-Lifshitz gravity can be used as an effective theory framework to describe the fractional quantum Hall effect, particularly at the low energy (hydrodynamic) regime. (2+1)-dimensional Hořava-Lifshitz gravity can be directly used to write down some FPD invariant effective actions, similar to what is done in $[31,61,66]$, while (3+1)-dimensional Hořava-Lifshitz gravity can serve as a holographic dual description. Although both Hořava-Lifshitz gravity and the non-relativistic quantum field theory for quantum Hall effects [60] (section 2) are FPD invariant, their field contents and the FPD of the fields are different. The foundation of the Hořava-Lifshitz formalism for quantum Hall effects is the map between the field contents and a matching of their FPD on both sides. This is one of the main results of this paper, given in (3.38)-(3.45). We originally derive this map as a holographic dictionary (section 3), the key component in any holographic duality. But it is worthy to emphasize that it can also exist independently from holography, since it is entirely written in $(2+1)$-dimensional field language. Thus it 
can be equally applies to (2+1)-dimensional Hořava-Lifshitz gravity as an non-holographic effective theory (more in the traditional sense) for quantum Hall effects.

We further derive a non-relativistic FPD invariant Chern-Simons low energy effective action for the fractional quantum Hall effect using Hořava-Lifshitz Holography with nondynamical Chern-Simons terms (section 4). It produces physical results in agreement with previous results obtained via other methods, for example, through Newton-Cartan formalism [61]. It encodes many universal geometric properties such as the shift and Hall viscosity. Using our results, we further clarify a mystery about the relation between Hall viscosity $\eta_{H}$ and angular momentum density $\ell$ :

$$
\eta_{H}=-\frac{1}{2} \ell
$$

This relation has been well established in the context of quantum Hall effects using other methods [29, 30, 47, 49], but not confirmed from the Chern-Simons effective theory as far as we know, including in holography [139-147]. ${ }^{4}$ We distinguish two types of angular momentum densities from the Chern-Simons effective theory and show that how the above relation arises (section 5). This inspires us to interpret the velocity field $v^{i}$ commonly appearing in quantum Hall effective field theory $\left(v^{i}\right.$ is related to torsion in the NewtonCartan formalism $[61,94,95]$ and to the shift function $N^{i}$ in our Hořava-Lifshitz formalism) as guiding center velocity field whose conjugate quantity is the guiding center momentum density, in conjecture with Haldane's geometric formalism [18, 50-52] of the fractional quantum Hall effect.

Notations: the field theory lives in a $(2+1)$-dimensional spacetime, with coordinates labeled by time $t$ and two spatial directions $\vec{x}=(x, y)$. The bulk radial coordinate is labeled by $r$, with the boundary located at $r=0$. Volume elements are $d^{3} x=d t d^{2} \vec{x}$ and $d^{4} x=$ $d^{3} x d r$. Indices $i, j, k$ and $l$ run through $x$ and $y$ (boundary spatial coordinates); $I, J$ and $K$ run through $x, y$ and $r$ (bulk spatial coordinates); $\mu$ and $\nu$ run through $t, x$ and $y$ (boundary spacetime coordinates); $M, N$ and $P$ run thought all four bulk coordinates. We use $\varepsilon$ with upper or lower indices to denote Levi-Civita tensors (with the corresponding determinant of the metric built in) and $\epsilon$ with indices Levi-Civita symbol (whose components are \pm 1 or 0 ); while $\epsilon$ appears with no index is the infinitesimal book-keeping parameter for the derivative expansion in late sections. We use $a$ and $b$ as the vielbein frame indices on the 2-dimensional manifold spanned by $x^{i}$, i.e. labeling the directions in the tangent space of $x$ and $y$, while $A$ and $B$ are the 3 -dimensional counterpart in the tangent space of $x, y$ and $r$. A "-" on a bulk field indicates it is the near-boundary $(r \rightarrow 0)$ independent leading order term of the corresponding bulk field. We will use the symbol " $\Rightarrow$ " to denote the near boundary behavior, i.e. the $r \rightarrow 0$ limit, where we keep the finite and possibly singular terms and drop the sub-leading terms that are vanishing in this limit.

\footnotetext{
${ }^{4}$ So far as we know, most holographic studies on Hall viscosity [139-141, 143, 144] assume no background magnetic field but finite temperature. Thus the results are for thermal Hall viscosity, not in the context of quantum Hall effects. An exception is [148], where vorticity is present.
} 


\section{A non-relativistic field theory in $2+1$ dimensions}

\subsection{Microscopic action and local symmetries}

The microscopic action that defines the quantum field theory for the quantum Hall effect in flat spacetime that we will study in this paper is given by

$$
S_{\mathrm{NR}}=\int d^{3} x\left\{\frac{i}{2}\left[\psi^{\dagger}\left(D_{t} \psi\right)-\left(D_{t} \psi\right)^{\dagger} \psi\right]-\frac{\delta^{i j}}{2 m}\left(D_{i} \psi\right)^{\dagger}\left(D_{j} \psi\right)+\frac{\mathrm{g} B}{4 m} \psi^{\dagger} \psi+\text { interactions }\right\},
$$

with $D_{\mu}=\partial_{\mu}-i A_{\mu}$ and the magnetic field $B=\epsilon^{i j} \partial_{i} A_{j}$. $\mathrm{g}$ is the gyromagnetic factor. To analyze local spacetime symmetries, we need to couple it to curved spacetime.

First, let us define the vielbein $e_{i}^{a}$ associated with the 2-dimensional spatial metric $g_{i j}$ :

$$
g_{i j}=\delta_{a b} e_{i}^{a} e_{j}^{b}, \quad g^{i j}=\delta_{a b} e^{a i} e^{b j},
$$

where $a, b=1,2$ are vielbein indices and $\delta_{a b}=(+,+)$ is the flat 2-dimensional Euclidean metric. $\epsilon_{a b}$ is the totally anti-symmetric Levi-Civita symbol and $\varepsilon^{i j}=\epsilon^{i j} / \sqrt{g}$ is the LeviCivita tensor associated with the metric $g_{i j}$, and $g=\operatorname{det}\left(g_{i j}\right)$. The U(1) spin connection vector $\omega_{\mu}=\left(\omega_{t}, \omega_{i}\right)$ is defined as

$$
\begin{aligned}
\omega_{t} & =\frac{1}{2} \epsilon_{a b} e^{a k} \partial_{t} e_{k}^{b}, \\
\omega_{i} & =\frac{1}{2}\left(\epsilon_{a b} e^{a k} \partial_{i} e_{k}^{b}-\varepsilon^{j k} \partial_{j} g_{k i}\right) .
\end{aligned}
$$

It transforms under diffeomorphism and Weyl transformation (to be introduced below) as

$$
\begin{aligned}
& \delta \omega_{t}=\xi^{\mu} \partial_{\mu} \omega_{t}+\omega_{\mu} \partial_{t} \xi^{\mu}+\frac{1}{2} \varepsilon^{j k} \partial_{j}\left(g_{k l} \partial_{t} \xi^{l}\right), \\
& \delta \omega_{i}=\xi^{\mu} \partial_{\mu} \omega_{i}+\omega_{k} \partial_{i} \xi^{k}-\varepsilon^{j k} g_{k i} \partial_{j} \sigma
\end{aligned}
$$

The scalar curvature of the 2-dimensional Euclidean space is $\mathcal{R}^{(2)}=\varepsilon^{i j} \partial_{i} \omega_{j}$ and transforms as a scalar under diffeomorphism. The magnetic field is now defined as $B=\varepsilon^{i j} \partial_{i} A_{j}$ in curved spacetime.

We choose microscopic action of the non-relativistic field theory in curved spacetime to be $S_{\mathrm{NR}}=S_{0}+S_{\text {int }}$ with

$$
S_{0}=\int d^{3} x \sqrt{g} e^{-\Phi}\left\{\frac{i}{2} e^{\Phi}\left(\psi^{\dagger}\left(D_{t} \psi\right)-\psi\left(D_{t} \psi\right)^{\dagger}\right)-\frac{1}{2 m}\left(g^{i j}+i \varepsilon^{i j}\right)\left(D_{i} \psi\right)^{\dagger}\left(D_{j} \psi\right)\right\},
$$

where the covariant derivative is

$$
\begin{aligned}
& D_{t}=\partial_{t}-i A_{t}+i s \omega_{t}-i \frac{(\mathrm{g}-2) e^{-\Phi}}{4 m}\left[B+(1-s) \mathcal{R}^{(2)}\right], \\
& D_{i}=\partial_{i}-i A_{i}+i s \omega_{i},
\end{aligned}
$$

where $s$ is intrinsic spin of $\psi$ (for spin-polarized electrons, i.e. components of Dirac spinor in vacuum, $s=1 / 2$ ). It is easy to see that $S_{0}$ goes back to the flat-spacetime action introduced at the beginning of this section. Possible choices for the interaction action $S_{\text {int }}$ include 4 -fermion interaction and Coulomb interaction. Our choice of $D_{t}$ such that 
$(\mathrm{g}-2) B / 4 m$ appears together with $A_{t}$ and $g^{i j}+i \varepsilon^{i j}$ appears in a combination in the standard Pauli form has been employed, for example, in [149]. This is different from [60], where $g^{i j}+i \frac{\mathrm{g}}{2} \varepsilon^{i j}$ appears together.

Under diffeomorphism $\delta t=-\xi^{t}(t), \delta x^{i}=-\xi^{i}(t, \vec{x})$, the action (2.7) is invariant up to boundary terms if the fields transform as following:

$$
\begin{aligned}
\delta_{\xi} \psi & =\xi^{\mu} \partial_{\mu} \psi \\
\delta_{\xi} A_{t} & =\xi^{\mu} \partial_{\mu} A_{t}+A_{\mu} \partial_{t} \xi^{\mu}-\frac{\mathrm{g}-2 s}{4} \varepsilon^{j k} \partial_{j}\left(g_{k l} \partial_{t} \xi^{l}\right)-\frac{\mathrm{g}-2}{4} \varepsilon^{i j}\left[\partial_{i} \log \left(m e^{\Phi}\right)\right] g_{j k} \partial_{t} \xi^{k} \\
\delta_{\xi} A_{i} & =\xi^{\mu} \partial_{\mu} A_{i}+A_{k} \partial_{i} \xi^{k}+m e^{\Phi} g_{i k} \partial_{t} \xi^{k} \\
\delta_{\xi} \Phi & =\xi^{\mu} \partial_{\mu} \Phi-\partial_{t} \xi^{t} \\
\delta_{\xi} e_{i}^{a} & =\xi^{\mu} \partial_{\mu} e_{i}^{a}+e_{k}^{a} \partial_{i} \xi^{k} \\
\delta_{\xi} g_{i j} & =\xi^{\mu} \partial_{\mu} g_{i j}+g_{i k} \partial_{j} \xi^{k}+g_{j k} \partial_{i} \xi^{k} \\
\delta_{\xi} m & =\xi^{\mu} \partial_{\mu} m \\
\delta_{\xi} g & =\xi^{\mu} \partial_{\mu} g \\
\delta_{\xi} s & =\xi^{\mu} \partial_{\mu} s
\end{aligned}
$$

Here we have assumed that the parameter $m, \mathrm{~g}$ and $s$ can also be external scalar fields whose values can vary at different spacetime points. This is usually true in the materials due to the presence of the medium. For example, elections' interaction with the lattice and medium around can not only shift the values of these parameter from their vacuum values, but also make them vary by positions. In this sense, the action shall be viewed as a renormalized effective action with effective mass $m$ etc [150].

So far we have not specified the precise meaning of $\psi$, or whether it is bosonic or fermionic. We want to keep it as general as possible. It just represents an underlying microscopic field degree of freedom which is to be path-integrated out when computing the effective action. It can be the electrons as well as other composite particles. Recently, [151] uses a similar action with the same coupling to gravity via spin connection for both composite fermion [4] and boson [3] theories to modify the conventional flux attachment procedure and derive the Wen-Zee shift and Hall viscosity. Our $\psi$ can be viewed in the same way, to denote the composite bosons or fermions, with corresponding interaction term $S_{\text {int }}$. In this case $s$ will be the topological spin of the composite particles, rather than the intrinsic or renormalized spin of the electrons. We will compare our results with those of [151] in section 5.4.

Under local anisotropic Weyl transformation $\sigma=\sigma(t, \vec{x})$, the action $(2.7)$ is invariant if the fields transform as

$$
\begin{aligned}
\delta_{\sigma} \psi & =-\sigma \psi, \\
\delta_{\sigma} \Phi & =-z \sigma, \\
\delta_{\sigma} e_{i}^{a} & =\sigma e_{i}^{a}, \\
\delta_{\sigma} g_{i j} & =2 \sigma g_{i j}, \\
\delta_{\sigma} A_{t} & =0, \\
\delta_{\sigma} A_{i} & =(1-s) \varepsilon^{j k} g_{k i} \partial_{j} \sigma, \\
\delta_{\sigma} m & =(z-2) \sigma m,
\end{aligned}
$$


The form of $S_{\text {int }}$ will be chosen to respect the above symmetries as well. [73] contains a brief discussion on $S_{\text {int }}$ in curved space. The form of $S_{\text {int }}$ for quantum Hall effects is typically bi-local:

$$
S_{\mathrm{int}}=-\frac{1}{2} \iint d^{3} x_{1} d^{3} x_{2} \sqrt{g\left(x_{1}\right) g\left(x_{2}\right)} e^{-\Phi\left(x_{1}\right)-\Phi\left(x_{2}\right)} \psi^{\dagger}\left(x_{1}\right) \psi\left(x_{1}\right) V\left(x_{1}, x_{2}\right) \psi^{\dagger}\left(x_{2}\right) \psi\left(x_{2}\right) .
$$

The form of $V\left(x_{1}, x_{2}\right)$ shall be chosen to respect the above symmetries. We will not discuss how to do this. One point we want to highlight is that, choosing different $V\left(x_{1}, x_{2}\right)$ may or may not introduce additional scales into the problem through its coupling coefficients. For example, if $V\left(x_{1}, x_{2}\right) \sim \lambda_{4 \mathrm{~F}} \delta^{(3)}\left(x_{1}-x_{2}\right)$ up to an inverse measure, the interaction becomes the contact 4-Fermi interaction [60, 114]:

$$
S_{\mathrm{int}}=-\frac{\lambda_{4 \mathrm{~F}}}{2} \int d^{3} x \sqrt{g} e^{-\Phi}\left(\psi^{\dagger} \psi\right)^{2} .
$$

Under Weyl transformation,

$$
\delta \lambda_{4 \mathrm{~F}}=-(z-2) \sigma \lambda_{4 \mathrm{~F}} .
$$

For $z=2$ it preserves the scale invariance, same as $m$ does. Another way to look at it is, $\lambda_{4 \mathrm{~F}}$ does not have a length scale associated with it when $z=2$ (i.e. dimensionless and marginal). ${ }^{5}$ In this case, $\lambda_{4 \mathrm{~F}}$ can still run logarithmically through quantum renormalization [152], but at the LLL projection ( $m \rightarrow 0$ limit) which is the most interesting case for us, this running will disappear. The interactions can also be introduced through auxiliary fields, such as that illustrated in [59] for Yukawa interaction. Other interactions, such as Coulomb and Yukawa interactions, usually have some dimensionful couplings. Once being included, these couplings will introduce additional length scales into the theory. Their Weyl transformations can be dealt in a similar fashion as for $m$ and $\lambda_{4 \mathrm{~F}}$, although their forms will be very different.

Without the interaction $S_{\text {int }}, m$ and $\Phi$ only appear together as a combination $m e^{\Phi}$ in $S_{0}$, which scales under Weyl transformation by $-2 \sigma$. In this case, talking the Weyl scaling of $m$ and $\Phi$ separately does not make much sense since one can always shift part of the scaling from one to the other, or equivalently, define $z=2$ and $\delta_{\sigma} m=0$. In other words, for free non-relativistic fields, $z$ always equals to 2 , and there is no Lifshitz scaling. This changes when the interactions such as that in $S_{\text {int }}$ is turned on, because now $\Phi$ appears separately in the interactions (for example, as part of the measure $e^{-\Phi}$ ), thus the Weyl scaling of $m$ and $\Phi$ can no longer be shifted between each other. In this case we can have $z \neq 2$ Lifshitz scaling physically. The fact that $m e^{\Phi}$ appears as a combination in $S_{0}$ is an accident of the free theory. Since we are considering interacting theories in general, we will always think that $m$ and $\Phi$ appear separately

We notice that $\mathrm{g}=2$ and $s=1$ are special values when the symmetry transformations are particularly simple and all the parity-violating terms are gone. This is also noticed in [60] and it corresponds to the parity-preserving case considered in early works of [59, 114, 115, 117]. Hence the holographic dictionary we are going to build will inherit this feature and goes back to that of [117] in this special case.

\footnotetext{
${ }^{5} \psi \sim[\text { length }]^{-1}, t \sim[\text { length }]^{z}$. Then $\int d^{3} x \sqrt{g} e^{-\Phi}\left(\psi^{\dagger} \psi\right)^{2} \sim[\text { length }]^{z-2}$. Thus $\lambda_{4 \mathrm{~F}} \sim[\text { length }]^{2-z}$.
} 
The action is also invariant under arbitrary shifts of parameters $\mathrm{g}$ and $s$ if the gauge field is shifted in the following way as well:

$$
\begin{aligned}
& \Delta A_{t}=\omega_{t} \Delta s-\frac{e^{-\Phi}}{4 m}\left\{\left[B+(1-s) \mathcal{R}^{(2)}\right] \Delta \mathrm{g}-\mathcal{R}^{(2)}(\mathrm{g}-2+\Delta \mathrm{g}) \Delta s\right\}, \\
& \Delta A_{i}=\omega_{i} \Delta s .
\end{aligned}
$$

This is essentially a field redefinition of $A_{\mu}$. It reflects the fact that in the microscopic action (2.7), g and $s$ only appear in the combination

$$
\begin{aligned}
& \tilde{A}_{t}=A_{t}-s \omega_{t}+\frac{(\mathrm{g}-2) e^{-\Phi}}{4 m}\left[B+(1-s) \mathcal{R}^{(2)}\right], \\
& \tilde{A}_{i}=A_{i}-s \omega_{i}
\end{aligned}
$$

Thus after path-integrating out the microscopic field $\psi$, in the effective action, $\mathrm{g}$ and $s$ will still only appear in the above combination of $\tilde{A}_{\mu}$, not separately. This imposes another useful constraint on the possible forms of the effective action. The combination $\tilde{A}_{\mu}$ transforms under diffeomorphism and Weyl transformation as

$$
\begin{aligned}
& \delta \tilde{A}_{t}=\xi^{\mu} \partial_{\mu} \tilde{A}_{t}+\tilde{A}_{\mu} \partial_{t} \xi^{\mu}-\frac{1}{2} \varepsilon^{i j} \partial_{i}\left(g_{j k} \partial_{t} \xi^{k}\right), \\
& \delta \tilde{A}_{i}=\xi^{\mu} \partial_{\mu} \tilde{A}_{i}+\tilde{A}_{k} \partial_{i} \xi^{k}+m e^{\Phi} g_{i k} \partial_{t} \xi^{k}+\varepsilon^{j k} g_{k i} \partial_{j} \sigma .
\end{aligned}
$$

The covariant derivative can be written as $D_{\mu}=\partial_{\mu}-i \tilde{A}_{\mu}$.

There are two local $\mathrm{U}(1)$ symmetry transformations under which the action is invariant. One is the local gauge transformation for the gauge field $A_{\mu}, \Lambda=\Lambda(t, \vec{x})$ :

$$
\delta A_{\mu}=\delta \tilde{A}_{\mu}=-\partial_{\mu} \Lambda
$$

with a shift of the phase factor $\psi \rightarrow e^{-i \Lambda} \psi$. The other is a local rotation of the vielbein frame, $\delta e_{i}^{a}=\Lambda_{b}^{a} e_{i}^{b}, \Lambda_{b}^{a}=\theta(t, \vec{x}) \epsilon_{b}^{a}$ :

$$
\delta \omega_{\mu}=-\partial_{\mu} \theta, \quad \delta \tilde{A}_{\mu}=s \partial_{\mu} \theta .
$$

This rotation corresponds to a spin rotation of the field $\psi \rightarrow e^{i s \theta} \psi$.

The effective action (strictly speaking the logarithm of the generating functional) $\mathcal{W}$ is a functional of $\Phi, g_{i j}, A_{\mu}, m, \mathrm{~g}, s$ among other external fields and parameters, defined after the path integral over $\psi$ :

$$
\mathcal{W}\left[\Phi, g_{i j}, A_{\mu}, \ldots ; m, \mathrm{~g}, s, \ldots\right]=-i \log \int \mathcal{D} \psi \mathcal{D} \psi^{\dagger} e^{i\left(S_{0}+S_{\mathrm{int}}\right)} .
$$

The expectation values of conserved current $J^{\mu}$, stress tensor $T^{i j}$ and energy density $\mathcal{E}^{0}$ can then be obtained as the following:

$$
\delta \mathcal{W}=\int d t d^{2} \vec{x} \sqrt{g} e^{-\Phi}\left\{\left\langle J^{\mu}\right\rangle \delta A_{\mu}+\frac{1}{2}\left\langle T^{i j}\right\rangle \delta g_{i j}+\left\langle\mathcal{E}^{0}\right\rangle \delta \Phi\right\} .
$$

The first goal of this paper is to find a holographic dictionary that will lead to the gravity dual description of the effective action $\mathcal{W}\left[\Phi, g_{i j}, A_{\mu}, \ldots ; m, \mathrm{~g}, s, \ldots\right]$. 


\subsection{Comments on coupling to curved spacetime}

It is necessary to note here that the way to couple a flat-spacetime action to curved spacetime is not unique. The curved-spacetime action (2.7) we choose here is slightly different from that in [60], where a minimal coupling is chosen. The differences are the form of $D_{t}$ and the position of g. There are two reasons that motivate us to choose the present form. We choose $\mathrm{g} B$ term to appear side-by-side with $A_{t}$ in $D_{t}$ to reflect the same fact in flat-spacetime action, where this is not manifest in [60] and complicated field redefinitions are needed if one wants to shift g. Consequently, the diffeomorphism of $A_{t},(2.11)$ is a little different than [60], and the relationship between momentum density $p^{i}$ and conserved current are modified as well. Following the same procedure as in [60], we can derive the relation for our current case:

$$
\left\langle p^{i}\right\rangle=m\left\langle J^{i}\right\rangle-\varepsilon^{i j} \partial_{j}\left(\frac{\mathrm{g}-2 s}{4} e^{-\Phi}\left\langle J^{t}\right\rangle\right)+\frac{\mathrm{g}-2}{4} e^{-\Phi}\left\langle J^{t}\right\rangle \varepsilon^{i j} \partial_{j} \log \left(m e^{\Phi}\right) .
$$

We also add $\mathcal{R}^{(2)}$ to $D_{t}$ to simplify the Weyl transformation of $A_{t},(2.23)$ so that it is identically zero. This simplifies the conformal Ward identity:

$$
\left\langle\mathcal{E}^{0}\right\rangle=\frac{1}{2} g_{i j}\left\langle T^{i j}\right\rangle-\frac{1-s}{2} e^{\Phi} \varepsilon^{i j} \nabla_{i}\left(e^{-\Phi}\left\langle J_{j}\right\rangle\right)
$$

when $z=2$. Here $\nabla_{i}$ is the covariant derivative compatible with metric $g_{i j}: \nabla_{k} g_{i j}=0$. Thus our version of the curved spacetime action is more toward the conformal coupling rather than the minimal coupling in [60]. Following the procedures in [60], one can derive all Ward identities for energy density, stress tensor and conserved current using the local symmetries discussed in the previous subsection.

\subsection{Global time and relationship to Newton-Cartan geometry}

Here we briefly address the issue related to global time, which is a hall-mark of nonrelativistic theories. A more comprehensive discussion has already been presented in [60]. We outline some of the key points here for self-containedness of later discussions. In the above discussion, energy flux $\mathcal{E}^{i}$ and its source (let us call it $C_{i}$ ) are missing. [60] shows that $C_{i}$ can be introduced to the formalism by modifying spatial quantities like $\partial_{i}$ and $D_{i}$ to $\tilde{\partial}_{i}=\partial_{i}+C_{i} \partial_{t}$ and $\tilde{D}_{i}=D_{i}+C_{i} D_{t}$. The diffeomorphism of $C_{i}$ is

$$
\delta C_{i}=\xi^{\mu} \partial_{\mu} C_{i}+C_{k} \tilde{\partial}_{i} \xi^{k}-\tilde{\partial}_{i} \xi^{t}
$$

and $n_{\mu}=\left(e^{-\Phi},-e^{-\Phi} C_{i}\right)$ transforms as a Lorentz vector. $C_{i}$ is invariant under local Weyl transformation. When

$$
\varepsilon^{i j}\left(\partial_{i}+C_{i} \partial_{t}\right) C_{j}=0, \quad \text { i.e. } \quad n \wedge \mathrm{d} n=0,
$$

there exist sets of global time coordinates (GTCs) in which $C_{i}=0$. This is generally required by the causality of non-relativistic theories. Diffeomorphisms that satisfy $\partial_{i} \xi^{t}=0$ (which keeps $C_{i}=0$ ) are the transformations between different GTCs. This also implies that in GTCs energy is globally conserved as time evolves and there is no notion of energy 
flux needed. In our discussion of the field theory, we have set $C_{i}=0$ and $\partial_{i} \xi^{t}=0$ throughout. This means that we have been assuming the existence of GTCs and work only in them. Since we are interested in non-relativistic field theories, this is reasonable and economical, and we will work in this way throughout the rest of the paper. We will leave the study of holographic dual to the case when $C_{i} \neq 0$ or even $n \wedge \mathrm{d} n \neq 0$ to the future.

$[94,95]$ and [60] show that for the case $n \wedge \mathrm{d} n=0$ (of which $C_{i}=0$ is a special case), the geometry can be covariantly described by Newton-Cartan geometry, with or without torsion. However, we want to emphasize that it is not always necessary to introduce such a formalism. One could just work with the spacetime symmetries (2.10)-(2.15) and (2.19)(2.25) directly with all those anomalous terms in the transformation rules. This is tabbed as "non-covariant" approach in [60]. The maths is usually cumbersome and laborious, but direct, and one gets all the physical results at the end. The Newton-Cartan geometry is a convenient way to covariantize the formalism, while other equivalent ways exist as well, with different notions of covariance and different structures in the formalisms. Since we assume the existence of GTCs, another convenient covariant formalism is Hořava-Lifshitz gravity. It has already been employed in [66]. The notion of covariance and metric and connection structures of Hořava-Lifshitz gravity are closer to those of relativistic gravity theories than to Newton-Cartan geometry, with additional assumption of the existence of global time that breaks the Lorentz symmetry between time and space. Since relativistic holography has been very well studied, given its similarity to relativistic gravity theory, Hořava-Lifshitz gravity is a natural choice as the covariant formalism we will use to covariantize the geometry of the non-relativistic field theory discussed in this section, because the generalization to the holographic bulk is ready $[117,118]$. The holographic dual we are studying here is an extension of [117]'s to parity violating cases, and its map to the boundary, which is the main results of our paper, can be viewed as another covariant description of the geometry of the non-relativistic field theory using Hořava-Lifshitz gravity, parallel to the popular Newton-Cartan formalism.

\section{Dual gravity theory in $3+1$ dimensional bulk}

\subsection{Bulk fields and their diffeomorphism}

The bulk theory, Hořava-Lifshitz gravity, assumes the existence of global time coordinates. The foliation of global time is preserved under diffeomorphism $x^{M}=-\hat{\xi}^{M}$ with the constraint

$$
\partial_{I} \hat{\xi}^{t}=0, \quad \text { i.e. } \quad \hat{\xi}^{M}=\left(\hat{\xi}^{t}(t), \hat{\xi}^{I}\left(t, x^{I}\right)\right) .
$$

This is the bulk counterpart of the condition $\partial_{i} \xi^{t}=0$ in the non-relativistic field theory discussed in the previous section, now extended to include the bulk radial direction as well.

The graviton sector of the bulk theory consists of a lapse function $N$, a shift function $N_{I}=\left(N_{i}, N_{r}\right)$ and a spatial metric $G_{I J}$. We define $G^{I J}$ is the matrix inverse of $G_{I J}$ and the index of the shift function $N_{I}$ is raised and lowered using $G^{I J}$ and $G_{I J}$. The bulk vielbein associated with $G_{I J}$ is $E_{I}^{A}$, with $G_{I J}=\delta_{A B} E_{I}^{A} E_{J}^{B}$. They transform under 
diffeomorphism as

$$
\begin{aligned}
\delta N & =\hat{\xi}^{M} \partial_{M} N+N \partial_{t} \hat{\xi}^{t} \\
\delta N_{I} & =\hat{\xi}^{M} \partial_{M} N_{I}+N_{I} \partial_{t} \hat{\xi}^{t}+N_{K} \partial_{I} \hat{\xi}^{K}+G_{I K} \partial_{t} \hat{\xi}^{K} \\
\delta G_{I J} & =\hat{\xi}^{M} \partial_{M} G_{I J}+G_{I K} \partial_{J} \hat{\xi}^{K}+G_{J K} \partial_{I} \hat{\xi}^{K} \\
\delta E_{I}^{A} & =\hat{\xi}^{M} \partial_{M} E_{I}^{A}+E_{K}^{A} \partial_{I} \hat{\xi}^{K}
\end{aligned}
$$

There is a bulk $\mathrm{U}(1)$ gauge field $V_{M}$ which transforms as

$$
\begin{aligned}
\delta V_{t} & =\hat{\xi}^{M} \partial_{M} V_{t}+V_{M} \partial_{t} \hat{\xi}^{M}, \\
\delta V_{I} & =\hat{\xi}^{M} \partial_{M} V_{I}+V_{K} \partial_{I} \hat{\xi}^{K}
\end{aligned} .
$$

and a scalar $\phi$ which transforms as

$$
\delta \phi=\hat{\xi}^{M} \partial_{M} \phi
$$

The rotations of the vielbein frame is

$$
\delta E_{I}^{A}=\hat{\Lambda}_{B}^{A} E_{I}^{B}, \quad \hat{\Lambda}_{B}^{A}=-\hat{\Lambda}_{A}^{B},
$$

where the anti-symmetric $\hat{\Lambda}_{B}^{A}$ are the generators of rotations. The above transformations are all infinitesimal transformations. The gauge transformation for $V_{M}$ is

$$
\delta V_{M}=-\partial_{M} \hat{\Lambda}
$$

We put a "^" on every bulk gauge parameter to distinguish it from the corresponding one in the field theory. The bulk action shall be invariant under all the above transformations.

\subsection{Gauge conditions and residual gauge transformations}

Next, we choose gauge conditions for fields in the bulk. The time-like boundary is located at $r=0$ and breaks the translational invariance along the spatial direction (denoted by the Poincaré radial coordinate $r$ here) perpendicular to the boundary. In the bulk, the isometry of the AdS-type geometry also naturally splits the 3-dimensional spatial manifold with metric $G_{I J}$ into the radial direction labeled by $r$ and the transverse directions (labeled by $x$ and $y$ ) with translational and $\mathrm{SO}(2)$ rotational symmetries. These introduce a codimension one foliation in the bulk labeled by $r=$ constant. We choose gauge conditions for the bulk fields that manifest this foliation:

$$
\begin{aligned}
E_{r}^{3} & =\Upsilon(r), \\
E_{x}^{3}=E_{y}^{3} & =0, \\
E_{r}^{1}=E_{r}^{2} & =0,
\end{aligned}
$$

Here we also fix the orientation of the vielbein frame in the tangent space such that 3direction is along $r$-direction and 1- and 2-directions are perpendicular to it. These give rise to the usual gauge conditions for the bulk metric: $G_{i r}=0$ and $G_{r r}=\Upsilon(r)^{2}$. Given an 
arbitrary choice of coordinates and frame to start with, these five gauge equations can be achieved by a combination of diffeomorphism and vielbein frame rotations within 1-3 and 2-3 planes, which fix $\xi^{I}$ and two of the three Euler angles of the rotations, related to $\Lambda_{3}^{1}$ and $\Lambda_{3}^{2}$, up to some residual gauge transformations to be discussed later. Similar gauge conditions have been used in [153]. In relativistic holography, another gauge condition $N_{r}=$ 0 is usually imposed, but here it can not be achieved, because this requires a diffeomorphism of $r$-dependent $\xi^{t}$, which is forbidden by (3.1). Thus we will not impose a gauge condition for $N_{r}$ in the bulk. For the bulk U(1) field, we choose the usual radial gauge condition

$$
V_{r}=0
$$

The above gauge conditions do not completely fix the gauge degrees of freedom. There are residual gauge transformations that leave these gauge conditions unchanged. The residual diffeomorphism that respect the gauge condition for metric $G_{i r}=0$ and $G_{r r}=$ $\Upsilon(r)^{2}$ is

$$
\left\{\begin{array}{l}
\hat{\xi}^{t}=\bar{\xi}^{t}(t) \\
\hat{\xi}^{i}=\bar{\xi}^{i}(x)+L \int d r \Upsilon(r) G^{i j}(x, r) \partial_{j} \bar{\sigma}(x), \\
\hat{\xi}^{r}=-\frac{L}{\Upsilon(r)} \bar{\sigma}(x)
\end{array}\right.
$$

where $x=(t, \vec{x})$ and $G^{i j}(x, r)$ is matrix inverse of $G_{i j}(x, r) . L$ is a length scale which will be set to the AdS radius later. However, the above diffeomorphism does not respect the gauge conditions for vielbein. It leaves $E_{r}^{3}$ invariant, but changes the others: $\delta E_{i}^{3}=-L \partial_{i} \bar{\sigma}$ and $\delta E_{r}^{a}=L \Upsilon(r) E^{a i} \partial_{i} \bar{\sigma}$. To compensate these shifts, we need to perform a residual rotation of the vielbein frame at the same time, with the following rotation parameters:

$$
\hat{\Lambda}_{3}^{a}=-L E^{a i}(x, r) \partial_{i} \bar{\sigma}(x) .
$$

Meanwhile, the rotation within 1-2 plane is still arbitrary: $\hat{\Lambda}_{2}^{1}=\hat{\Lambda}_{2}{ }_{2}(x, r)$. The residual diffeomorphism also changes $V_{r}$ by $\delta V_{r}=L \Upsilon(r) G^{i j} V_{i} \partial_{j} \bar{\sigma}$. To compensate this shift, the residual gauge transformation for the $\mathrm{U}(1)$ field is

$$
\hat{\Lambda}=\bar{\Lambda}(x)+L \int d r \Upsilon(r) G^{i j}(x, r) V_{i}(x, r) \partial_{j} \bar{\sigma}(x) .
$$

We shall note here that one is entitled to choose different gauge conditions in the bulk other than the ones we specify above. But we need that at least near the boundary the gauge conditions agree with the form specified here, because the near-boundary residual symmetry transformations and hence the holographic dictionary are built on the choice of the above gauge conditions near the boundary, and these gauge conditions do reflect the fact that the existence of the time-like boundary at $r=0$ breaks the isometry along $r$-direction. The consequences of this set of gauge conditions near the boundary is what we will explore next. 


\subsection{Near-boundary behaviors of fields and transformations}

Near the boundary $r=0$, we choose the background metric to have the asymptotic Lifshitz form $[117,134,153,154]$ in Poincaré coordinates: ${ }^{6}$

$$
d s^{2} \Rightarrow-\left(\frac{L}{r}\right)^{2 z} d t^{2}+\left(\frac{L}{r}\right)^{2}\left(d \vec{x}^{2}+d r^{2}\right)
$$

where $L$ is AdS radius. This implies $\Upsilon(r)=L / r$ in the gauge conditions. The nearboundary behaviors of the bulk graviton fields take the following form:

$$
\begin{aligned}
N & \Rightarrow\left(\frac{L}{r}\right)^{z}[\bar{N}(x)+O(r)], \\
N_{I} & \Rightarrow\left(\frac{L}{r}\right)^{2}\left[\bar{N}_{I}(x)+O(r)\right], \\
G_{i j} & \Rightarrow\left(\frac{L}{r}\right)^{2}\left[\bar{G}_{i j}(x)+O(r)\right], \\
E_{i}^{a} & \Rightarrow\left(\frac{L}{r}\right)\left[\bar{E}_{i}^{a}(x)+O(r)\right] .
\end{aligned}
$$

The asymptotic indices $z$ and 2 of $N$ and $G_{i j}$ are determined by the above background Lifshitz metric. The index of $N_{I}$ is in general not fully determined by the background metric, but also by other free parameters of Hořava-Lifshitz gravity, and thus can be tuned for a desired value. We choose the value to be 2 , for reasons to be explained later. The near-boundary behavior of the $\mathrm{U}(1)$ field is

$$
V_{\mu} \Rightarrow \bar{V}_{\mu}(x)+O(r)
$$

i.e. the asymptotic $r$ index is 0 for $V_{\mu}$. The reason for that is this index corresponds to the coefficient of Weyl parameter $\sigma$ in the global Weyl transformation in the dual field theory. For the U(1) field in the field theory, it does not transform under global Weyl transformation, i.e. the coefficient is 0 , thus we choose the $r$ index to be 0 in the bulk dual. This can always be achieved by tuning the parameters of the bulk theory. We choose the scalar to have the following asymptotic behavior:

$$
\phi \Rightarrow\left(\frac{L}{r}\right)^{\Delta_{\phi}}[\bar{\phi}(x)+O(r)]
$$

where $\Delta_{\phi}$ is related to the conformal dimension of the dual operator and can be tuned by the mass of the scalar in the bulk theory.

\footnotetext{
${ }^{6}$ This metric form shall be understood as the following asymptotic conditions for the background functions:

$$
N \Rightarrow\left(\frac{L}{r}\right)^{z}, \quad N_{I} \Rightarrow 0, \quad G_{I J}=\left(\frac{L}{r}\right)^{2} \delta_{I J}
$$

[117] has a more general setup that $N \Rightarrow(L / r)^{\gamma}$ with $\gamma \neq z$. For simplicity, we will assume $\gamma=z$ to keep our holographic map neat. $\gamma \neq z$ can always be achieved by tuning the asymptotic indices of scalar fields/components in the holographic map, at the price of making it appear more complicated, as shown in [117].
} 
Then near the boundary, the bulk residual diffeomorphism becomes

$$
\left\{\begin{array}{l}
\hat{\xi}^{t} \Rightarrow \bar{\xi}^{t}(t) \\
\hat{\xi}^{i} \Rightarrow \bar{\xi}^{i}(x)+\frac{1}{2} r^{2} \bar{G}^{i j}(x) \partial_{j} \bar{\sigma}(x)+O\left(r^{3}\right), \\
\hat{\xi}^{r} \Rightarrow-r \bar{\sigma}(x)
\end{array}\right.
$$

and the residual vielbein rotation and $\mathrm{U}(1)$ gauge transformation become

$$
\begin{aligned}
\hat{\Lambda}_{3}^{a} & \Rightarrow-r \bar{E}^{a i}(x) \partial_{i} \bar{\sigma}(x), \\
\hat{\Lambda} & \Rightarrow \bar{\Lambda}(x)+\frac{1}{2} r^{2} \bar{G}^{i j}(x) \bar{V}_{i}(x) \partial_{j} \bar{\sigma}(x)+O\left(r^{3}\right),
\end{aligned}
$$

where $\bar{G}^{i j}$ is the matrix inverse of $\bar{G}_{i j}$. To maintain the near-boundary asymptotic behavior of the bulk vielbein in (3.22), we need

$$
\hat{\Lambda}_{b}^{a} \Rightarrow \bar{\Lambda}_{b}^{a}(x)+O(r) .
$$

We will call fields with "-" boundary fields. $i, j, k$ and $l$ indices of the boundary fields are raised and lowered by $\bar{G}^{i j}$ and $\bar{G}_{i j}$, e.g. $\bar{E}^{a i}=\bar{G}^{i j} \bar{E}_{j}^{a}$.

From the near-boundary asymptotic behaviors of the bulk fields and the bulk diffeomorphism, we can work out the residual symmetry transformations of the boundary fields at the boundary $r=0$ :

$$
\begin{aligned}
\delta \bar{N} & =\bar{\xi}^{\mu} \partial_{\mu} \bar{N}+\bar{N} \partial_{t} \xi^{t}+z \bar{\sigma} \bar{N} \\
\delta \bar{N}_{i} & =\bar{\xi}^{\mu} \partial_{\mu} \bar{N}_{i}+\bar{N}_{i} \partial_{t} \bar{\xi}^{t}+\bar{N}_{k} \partial_{i} \bar{\xi}^{k}+\bar{G}_{i k} \partial_{t} \bar{\xi}^{k}+2 \bar{\sigma} \bar{N}_{i}, \\
\delta \bar{N}_{r} & =\bar{\xi}^{\mu} \partial_{\mu} \bar{N}_{r}+\bar{N}_{r} \partial_{t} \bar{\xi}^{t}+\bar{\sigma} \bar{N}_{r} \\
\delta \bar{G}_{i j} & =\bar{\xi}^{\mu} \partial_{\mu} \bar{G}_{i j}+\bar{G}_{i k} \partial_{j} \bar{\xi}^{k}+\bar{G}_{j k} \partial_{i} \bar{\xi}^{k}+2 \bar{\sigma} \bar{G}_{i j} . \\
\delta \bar{E}_{i}^{a} & =\bar{\xi}^{\mu} \partial_{\mu} \bar{E}_{i}^{a}+\bar{E}_{k}^{a} \partial_{i} \bar{\xi}^{k}+\bar{\sigma} \bar{E}_{i}^{a}+\bar{\Lambda}_{b}^{a} \bar{E}_{i}^{b} \\
\delta \bar{V}_{\mu} & =\bar{\xi}^{\nu} \partial_{\nu} \bar{V}_{\mu}+\bar{V}_{\nu} \partial_{\mu} \bar{\xi}^{\nu}-\partial_{\mu} \bar{\Lambda} \\
\delta \bar{\phi} & =\bar{\xi}^{\mu} \partial_{\mu} \bar{\phi}+\Delta_{\phi} \bar{\sigma} \bar{\phi}
\end{aligned}
$$

We define $\bar{\varepsilon}^{i j}$ is the Levi-Civita tensor associated with the boundary metric $\bar{G}_{i j}$. There are additional vectorial structures we can make up using the above boundary fields, whose near-boundary diffeomorphism transformations are collected in appendix B.

\subsection{Holographic dictionary}

Now we are in a position to map the boundary fields of the bulk gravity theory to the source fields of the field theory. The guiding principle is the matching of symmetry transformations of the fields on both sides. First, we map the symmetry transformation parameters, following the idea of [118]. The near-boundary residual diffeomorphism parameters $\bar{\xi}^{\mu}$ and $\bar{\sigma}$ of the bulk theory are mapped to the diffeomorphism and Weyl parameters $\xi^{\mu}$ and $\sigma$ in the field theory, while the residual gauge parameter $\bar{\Lambda}$ is mapped to the gauge parameter $\Lambda$.

It immediately follows that $\bar{G}_{i j}$ and $\bar{E}_{i}^{a}$ are mapped to $g_{i j}$ and $e_{i}^{a}$ in the field theory, since their diffeomorphism and Weyl transformations match perfectly. The vielbein frame 
rotation $\bar{\Lambda}_{b}^{a}(x)$ is mapped to that in the field theory which rotates the frame of $e_{i}^{a}$. The near-boundary diffeomorphism of $\bar{N}$ matches to $e^{-\Phi}$ perfectly as well. There is no obvious field theory dual for $\bar{N}_{r}$. In fact, in relativistic holography, its counterpart the bulk field $G_{t r}$ is usually set identically to zero by a gauge condition thus its boundary field $\bar{G}_{t r}$ is automatically vanishing. Here we do not gauge away the bulk field $N_{r}$ because of the nonrelativistic nature of the bulk gravity theory: $\partial_{I} \hat{\xi}^{t}=0$ which forbids this action. However, we are still allowed to set its boundary value $\bar{N}_{r}$ to zero as a boundary condition and this is indeed consistent (invariant) with respect to the residual diffeomorphism (3.31).

Now the only remaining part is to map $\bar{N}_{i}$ and $\bar{V}_{\mu}$ to $\tilde{A}_{\mu}$ in the field theory. None of their diffeomorphism (3.30) and (3.34) matches directly with the transformations (2.31) and (2.32) of $\tilde{A}_{\mu}$. Since both $\bar{V}_{\mu}$ and $\tilde{A}_{\mu}$ are U(1) gauge fields, and the residual gauge transformation $\bar{\Lambda}(x)$ in (3.27) is already mapped to the gauge transformation of $\tilde{A}_{\mu}$, it is natural to primarily map $\bar{V}_{\mu}$ to $\tilde{A}_{\mu}$, i.e. $\bar{V}_{\mu}=\tilde{A}_{\mu}+\ldots$, with some additional structures "..." in the map so that their diffeomorphism and Weyl transformation match perfectly. We have collected these additional structures and their diffeomorphism transformations in appendix B. For example, one of the structures we add to $\tilde{A}_{i}$ is the field theory dual of the near-boundary term $\bar{\phi} \bar{N}_{i} / \bar{N}$, whose diffeomorphism is given in (B.5). By requiring $\bar{G}_{i k} \partial_{t} \bar{\xi}^{k}$ term to cancel the $m e^{\Phi} g_{i k} \partial_{t} \xi^{k}$ term in (2.32) and the Weyl index to be zero, we can identify the map for the scalar: $\bar{\phi}=m$ with conformal dimension $\Delta_{\phi}=z-2$, which also matches that of $m$ 's in (2.25). The physical meaning of the bulk scalar $\phi$ is clear now: it is the bulk dual of the mass $m$. When $z=2$, it is consistent to set it to a constant in the bulk theory, i.e. just a parameter with no dynamics. When $z \neq 2$, we know in the field theory that $m$ is not scale-invariant (from its Weyl transformation) and it will have a non-trivial renormalization group (RG) flow from its UV value (or function) in (2.7) to some other value (function) in the IR. In this case, the dual bulk field $\phi$ can be dynamical and develop some non-trivial profile in the bulk, which is a geometrization of the RG flow of $m$ in the field theory. The other structures involving $\varepsilon^{i j}$ in appendix B are used to cancel the other anomalous terms $-\frac{1}{2} \varepsilon^{i j} \partial_{i}\left(g_{j k} \partial_{t} \xi^{k}\right)$ in (2.31) and $\varepsilon^{j k} g_{k i} \partial_{j} \sigma$ in (2.32).

The last question remaining is what $\bar{N}_{i}$ maps to in the field theory? So far every source field in the field theory action (2.7) has been mapped from the near-boundary fields of the dual gravity theory, and it seems there is no target image left for $\bar{N}_{i}$. Even though $\bar{N}_{i}$ appears as additional vectorial structures in the map between $\bar{V}_{\mu}$ and $\tilde{A}_{\mu}$, we can view this as primarily a one-to-one and onto map between $\bar{V}_{\mu}$ and $\tilde{A}_{\mu}$, so $\bar{N}_{i}$ itself is still unmapped. Alternatively, we can think the spatial components of this map maps a combination of $\bar{V}_{i}$ and $\bar{N}_{i}$ to $\tilde{A}_{i}$, which is a two-to-one map, thus we still need to map the other independent combination of $\bar{V}_{i}$ and $\bar{N}_{i}$ to something in the field theory. At this time, let us just assume that $\bar{N}_{i}$ maps to some field, called $-v_{i}$, in the field theory, whose transformation is exactly the same as $\bar{N}_{i}$ 's given in (3.30). We will discuss the consequence of this and the meaning of $v_{i}$ in detail in the next subsection and late sections. Here for the construction of holographic map, the presence of $\bar{N}_{i}$ is crucial, because without it we would not be able to add additional structures, as those shown in appendix B, to the map between $\bar{V}_{\mu}$ and $A_{\mu}$ to match their symmetry transformations on both sides. 
Before presenting the holographic map, we notice that the following modified definition for spin connection

$$
\begin{aligned}
& \omega_{t}^{\prime}=\omega_{t}+\frac{1}{2} \varepsilon^{i j} \partial_{i} v_{j}-\frac{1}{2} \varepsilon^{i j} v_{i} \partial_{j} \log \left(m e^{\Phi}\right) \\
& \omega_{i}^{\prime}=\omega_{i}+\frac{1}{2} \varepsilon^{j k} g_{i j} \partial_{k} \log \left(m e^{\Phi}\right)
\end{aligned}
$$

transforms as an exact Lorentz vector under diffeomorphism and invariantly under local Weyl transformation, i.e. same as $\bar{V}_{\mu}$ in (3.34). Thus we can always add $\omega_{\mu}^{\prime}$ to the map of $\bar{V}_{\mu}$ with an arbitrary coefficient (we call $s^{\prime}$ ) while still preserve the transformation properties of the map.

We now present the holographic dictionary. The maps for the fields are

$$
\begin{aligned}
\bar{N} & =e^{-\Phi}, \\
\bar{G}_{i j} & =g_{i j}, \\
\bar{E}_{i}^{a} & =e_{i}^{a}, \\
\bar{N}_{i} & =-v_{i}, \\
\bar{N}_{r} & =0 \\
\bar{V}_{t} & =\tilde{A}_{t}-s^{\prime} \omega_{t}^{\prime}-\frac{1}{2} m e^{\Phi} v^{2}-\frac{1}{2} \varepsilon^{i j} \partial_{i} v_{j}+\frac{1}{2} \varepsilon^{i j} v_{i} \partial_{j} \log \left(m e^{\Phi}\right), \\
\bar{V}_{i} & =\tilde{A}_{i}-s^{\prime} \omega_{i}^{\prime}+m e^{\Phi} v_{i}-\frac{1}{2} \varepsilon^{j k} g_{i j} \partial_{k} \log \left(m e^{\Phi}\right), \\
\bar{\phi} & =m,
\end{aligned}
$$

and the near-boundary index of $\phi$ to be

$$
\Delta_{\phi}=z-2
$$

Here $v^{2}=g^{i j} v_{i} v_{j}$. Since in the field theory we allow $\mathrm{g}$ and $s$ to be scalar functions which are Weyl invariant, they, and the new $s^{\prime}$, can also be dual to some bulk scalar fields with $r^{0}$ asymptotic behavior near the boundary. So far $s^{\prime}$ is just a parameter appearing in the map which is allowed by the symmetries. Its physical meaning in the context of fractional quantum Hall effect will be explained in section 5.4 when we compare our results with the composite fermion and boson theories.

There can be additional parameters in the field theory, particularly the couplings of interactions in $S_{\text {int }}$. They can be dual to additional bulk scalars, whose near-boundary behavior (conformal dimension or bulk mass) shall match the corresponding Weyl transformation in the field theory. For example, the 4-Fermi interaction coupling $\lambda_{4 \mathrm{~F}}$ in (2.26) shall dual to a bulk scalar with asymptotic $r^{z-2}$ leading order behavior near the boundary. When $z=2$, both $m$ and $\lambda_{4 \mathrm{~F}}$ are marginal by dimensional counting. If they are truly constant in a conformal field theory (i.e. no running through renormalization), then their bulk dual scalars can also be set to the same constants without dynamics. However, if they are renormalized [152], their bulk dual shall still be some dynamic fields that can develop some non-trivial profiles in the bulk to reflect this running of couplings. 


\subsection{Additional constraints}

Now let us discuss what $v_{i}$ (i.e. $-\bar{N}_{i}$ ) means and how we can deal with it. These are actually two separate questions. In relativistic holography, from the perspective of bottom-up approaches, because the full relativistic Poincaré symmetry is very powerful to limit the allowed bulk action terms that one can start with, the bulk dynamics is very restrictive. Typically, $\bar{N}_{i}$ is an independent boundary field and appears in the on-shell boundary action. On contrary, for a non-relativistic gravity theory, such as Hořava-Lifshitz gravity, the symmetry is less restrictive. Thus one has more freedom to add additional terms to the action with arbitrary coefficients. These coefficients act as external tunable parameters that one can use to fine-tune the bulk dynamics to the desired form that is appropriate for the problem in consideration. In this large landscape of bulk dynamics and possible forms of on-shell boundary action, $\bar{N}_{i}$ can behave quite differently, thus gives rises to different interpretations and treatments of its field theory dual $v_{i}$. There exist many different scenarios. There are certain scenarios in which the question of how to deal with $\bar{N}_{i}$ becomes trivial. Two examples are as following.

1. For some tactically constructed model bulk actions (possibly in the help of additional symmetries such as the $\mathrm{U}(1)_{\Sigma}$ symmetry of $\left.[132,133]\right)$ with certain choices of parameters, $\bar{N}_{i}$ (i.e. $-v_{i}$ ) may completely disappear after the holographic dictionary is applied. This possibility is considered first in [117].

2. For certain choices of the parameters of the bulk action the bulk equations of motion near the boundary may constrain $\bar{N}_{i}$ to be either vanishing or (local or non-local) functional of the other boundary fields, thus it is not independent. Similar phenomena are not unfamiliar in relativistic Lifshitz holography $[155,156]$, especially for $z>2$. Consequently, $v_{i}$ is a functional of other source fields such as $g_{i j}$ and $\Phi$. In these cases, we only need to find out what this functional is by solving the equations of motion. $\bar{N}_{i}$ is essentially mapped to other source fields by this functional. There is no additional independent source field $v_{i}$ appearing in the field theory through the holographic dictionary. In these cases, there is no puzzle of how to deal with $v_{i}$, although the question of how to interpret it still exists.

In the rest of this paper, we will focus on another possible scenario similar to the relativistic case, that is, when $\bar{N}_{i}$ is independent of the other boundary fields in the bulk theory, and after the holographic dictionary is applied, it still appears (as $\left.-v_{i}\right)$ in the effective action. We then need to find a way to interpret the meaning of $v_{i}$ and the operator $\hat{\mathcal{O}}^{i}$ it sources (if it does), and to find a way to determine it. We think this is the case most relevant to quantum Hall effects and later we will show that it does yield results in nice agreement with the quantum Hall literature.

\subsubsection{How not to interpret $v_{i}$}

First let us try to find out what could not be the possible interpretation of $v_{i}$. A reasonable speculation is that since it comes from $\bar{N}_{i}$, which is essentially the $t i$-component of the 
full bulk spacetime metric near the boundary, by analogy to the relativistic holographic dictionary, it sources energy flux $\mathcal{E}^{i}$ and/or momentum density $p^{i}$ of the field theory.

For energy flux $\mathcal{E}^{i}$, we have discussed in the previous section that it is sourced by the field $C_{i}$ in the field theory. But the diffeomorphism of $C_{i},(2.39)$ is different from $\bar{N}_{i}$ 's in (3.30). Thus $v_{i}$ can not be identified with the source field for energy flux. Furthermore, we have worked in GTCs throughout, in which $C_{i}=0$. In GTCs energy is globally conserved and there is no need for the notion of energy flux in the field theory we are considering. Even we want to match $v_{i}$ to $C_{i}$, it must be zero in GTCs. But $v_{i}=0$ is not invariant under residual diffeomorphism (3.30), thus can not be a valid map or constraint. On the other hand, from the holographic point of view, [117] has conjectured that the energy flux source $C_{i}$ (what is called $B_{i}$ there) is dual to what they call the field $P_{i}$ in the bulk, the $O\left(c^{2}\right)$ order of $t i$-component of the full spacetime Lorentz metric if the dependence on speed of light $c$ is restored: $G_{t I}=c^{2} P_{I}+N_{I}$. Diffeomorphism of $P_{I}$ involves explicitly $\partial_{I} \hat{\xi}^{t}$. The non-relativistic theory can be viewed as a limit of $c \rightarrow \infty$. For us, since we have restricted ourselves to GTCs for both field theory and its bulk dual, in which $\partial_{I} \hat{\xi}^{t}=0$ and $P_{I}$ vanishes identically, $v_{i}$ can not be related to energy flux.

How about momentum density $p^{i}$ ? The answer is again negative. As we discussed in the previous section, in the non-relativistic field theory, the momentum density is not an independent quantity, but rather expressed in terms of the conserved current as given in (2.37). Thus there is no need for a separate field in the field theory to source the momentum density $\left\langle p_{i}\right\rangle$. The only thing one need to do is to compute $\left\langle J^{\mu}\right\rangle$, then use (2.37) to calculate $\left\langle p^{i}\right\rangle$. Thus it is not necessary to have a separate source field $v_{i}$ to source momentum density.

\subsubsection{How not to deal with $v_{i}$}

In fact, an object similar to $v_{i}$ has appeared in the literature. In both [61] and [60] it is the so called "velocity vector" (also denoted by $v^{i}$ there) in the Newton-Cartan geometry which is employed to aid to construct the effective action of the field theory based on symmetries. This velocity vector essentially acts like a dual shift function for Newton-Cartan geometry, similar to the role of $N^{I}$ in our bulk theory. In fact if we raise $v_{i}$ 's index by $g^{i j}$, the resulting transformation for $v^{i}$ is

$$
\delta v^{i}=\xi^{\mu} \partial_{\mu} v^{i}+v^{i} \partial_{t} \xi^{t}-v^{k} \partial_{k} \xi^{i}-\partial_{t} \xi^{i}
$$

This is the same as the transformation rule for $v^{i}$ in [60,61]. The second term $v^{i} \partial_{t} \xi^{t}$ is absent in [61], because $\Phi=0$ and $\partial_{t} \xi^{t}=0$ there. Thus, we can say that our $v^{i}$ is essentially the same as that appears in $[60,61]$. (The same object appears in Lifshitz holography as well $[94,95]$.) In neither [61] nor [60] does $v^{i}$ act like a fundamental external source field. In [60] the introduction of $v^{i}$ to the field theory is to modify the seemingly non-covariant vector potential $A_{\mu}$ to be a Newton-Cartan covariant vector and thus to reformulate the effective action and Ward identities in a covariant manner. This is similar to what we are doing here: we can view the map (3.43) and (3.44) is a modification from the non-covariant $A_{\mu}$ (in aid of $v_{i}$ ) to the covariant vector $\bar{V}_{\mu}$, and the on-shell action calculated from the 
bulk dynamics automatically offers a covariant way to organize the effective action as a functional of the covariant sources $\bar{V}_{\mu}, \bar{N}, \bar{N}_{i}, \bar{G}_{i j}$ among others. The difference between our case and [60]'s is the notion of covariance - Lorentzian for us (strictly speaking Hořava, which is Lorentzian with $\partial_{i} \xi^{t}=0$ ) while Newton-Cartan for [60], but other than these technical details, the philosophy is the same. Thus we can identify our $v^{i}$ as the same $v^{i}$ appearing in $[60,61]$. Based on the fact that $v^{i}$ is only introduced formally and shall not affect the physics, [60] assumes that the effective action shall be independent of $v^{i}$ once it is written out explicitly in terms of the physical sources $\Phi, g_{i j}, A_{\mu}$ etc. However, effective action obtained from holography can rarely satisfy such a stringent constraint, so we assume our effective action will depend on $v^{i}$ in general, unless we impose an additional constraint besides the holographic map (3.38)-(3.45). This additional constraint is precisely the subject under discussion here.

On the other hand, [61] views $v^{i}$ as a dynamical field with respect to which the effective action is extremized. From the field theoretical point of view, $\mathcal{W}$ in $(2.35)$ is the extremum of another quasi-effective action $\mathcal{I}\left[v^{i}, A_{\mu}, g_{i j}, \Phi, \ldots\right]$ with respect to $v^{i}$. In other words, $v^{i}$ has been path-integrated out in $\mathcal{I}$ which can be classically done by imposing Euler-Lagrange equation for $v^{i}$. What is the origin of $v^{i}$ in field theory? It is possibly an auxiliary field introduced to facilitate path-integral for the microscopic field $\psi$. For example, in [61] it is shown to arise from the Hubbard-Stratonovich transformation for $D_{i} \varphi$, where $\varphi$ is the phase of $\psi$. After such a transformation, one can integrate out $\psi$ and $\psi^{\dagger}$ (i.e. $|\psi|$ and $\varphi$ ), leaving an quasi-effective action $\mathcal{I}$ which depends explicitly on $v^{i}$. The path-integral of $v^{i}$ remains to be carried out to yield the final answer to the effective action $\mathcal{W}$. This can be formally written as

$$
e^{i \mathcal{W}\left[A_{\mu}, g_{i j}, \Phi, \ldots\right]}=\int \mathcal{D} v^{i} e^{i \mathcal{I}\left[v^{i}, A_{\mu}, g_{i j}, \Phi, \ldots\right]}
$$

where

$$
e^{i \mathcal{I}\left[v^{i}, A_{\mu}, g_{i j}, \Phi, \ldots\right]}=\int \mathcal{D} \psi \mathcal{D} \psi^{\dagger} e^{i S^{\prime}\left[\psi, v^{i}, A_{\mu}, g_{i j}, \Phi, \ldots\right]}
$$

and $S^{\prime}\left[\psi, v^{i}, \ldots\right]$ is related to the original microscopic action $S[\psi, \ldots]$ by a HubbardStratonovich transformation. The path-integral over $v^{i}$ can be done classically by imposing the Euler-Lagrange equation for $v^{i}$

$$
\frac{\delta}{\delta v^{i}} \mathcal{I}\left[v^{i}, A_{\mu}, g_{i j}, \Phi, \ldots\right]=0
$$

and finding the extremum of $\mathcal{I}$.

In holographic approach, after calculating the bulk dynamics, we will end up with an on-shell action as a functional of the boundary fields: $\overline{\mathcal{I}}\left[\bar{N}_{i}, \bar{V}_{\mu}, \cdots\right]$. Here every thing is written in terms of the bulk theory language, and the near-boundary field $\bar{N}_{i}, \bar{V}_{\mu}$ and others are viewed as independent variables of the functional $\overline{\mathcal{I}}$. We denote the other boundary fields such as $\bar{N}, \bar{G}_{i j}$ etc as "...". Next we use our holographic dictionary (3.38)-(3.45) to write the action explicitly in terms of the physical sources $\Phi, g_{i j}, A_{\mu}$ and the extra field $v^{i}$, and identify this functional as $\mathcal{I}\left[v^{i}, A_{\mu}, g_{i j}, \Phi, \ldots\right]$ introduced before, whose independent variables are now $v^{i}$ and $A_{\mu}, g_{i j}$ and $\Phi$ :

$$
\overline{\mathcal{I}}\left[\bar{N}_{i}\left[v_{i}\right], \bar{V}_{\mu}\left[v_{i}, A_{\mu} \ldots\right], \cdots\right]=\mathcal{I}\left[v^{i}, A_{\mu}, g_{i j}, \Phi, \ldots\right] .
$$


Now if we naively apply the philosophy of [61], we can impose (3.48) as a constraint, find its solution $v_{\star}^{i}$, then the effective action

$$
\mathcal{W}\left[A_{\mu}, g_{i j}, \Phi, \ldots\right]=\mathcal{I}\left[v_{\star}^{i}, A_{\mu}, g_{i j}, \Phi, \ldots\right]
$$

From the holographic point of view, this approach seems fine, and we do get a result for $\mathcal{W}$, which is an effective action of a certain quantum field theory with the same nonrelativistic spacetime symmetries discussed in the previous section. But from the field theoretical point of view, is it the one for the field theory we discussed in the previous section which describes the quantum Hall effect? Not quite! A subtlety is that in [61] the path integral is not only carried out for $v^{i}$, but also for other auxiliary fields $\rho, \varphi$ and $a_{\mu}$. Thus even we are doing the same integral for $v^{i}$, we are not completely in parallel with [61]. A consequence is that our results obtained from this way will not have a welldefined projection to the lowest Landau level (LLL). This can be shown by a straightforward calculation which we will not present here. The projection to LLL is defined as the limit $\mathrm{g}=2$ and $m \rightarrow 0$, and the effective action $\mathcal{W}$ shall be regular in this limit. [60] has shown that field theories defined by microscopic actions such as (2.7) do have a smooth LLL projection. However, if we impose (3.48) as the additional constraint in holography, the resulting effective action is either singular or over-constrained in LLL projection. Thus it can not be a valid constraint. This choice is ruled out not by holographic considerations, but by a field theoretical consideration - the LLL projection, which is very special to the quantum Hall problem we are considering here. Had one considered a holographic dual for another quantum system, this constraint might be a viable one.

\subsubsection{Choice (I): a path integral constraint}

We have just argued that the path integral over $v^{i}$ does not work, but the idea of integrating out the extra fields still work. The key point is that we need to find out the right fields to integrate out. In addition to the requirement that it reproduces the right features of the quantum Hall problem, such as the LLL projection, a more fundamental criterion is that the path integral over these fields shall also be gauge and diffeomorphism invariant. More specifically, path integral over some fields is classically equivalent to imposing the Euler-Lagrange equations for those fields, similar to the extremum condition (3.48) for $v^{i}$, and these equations shall be invariant under gauge transformation and diffeomorphism. Instead of looking for such constraints for the field theory action $\mathcal{I}\left[v^{i}, A_{\mu}, g_{i j}, \Phi, \ldots\right]$, it is much easier to find ones for the holographic action $\overline{\mathcal{I}}\left[\bar{N}_{i}, \bar{V}_{\mu}, \cdots\right]$. Since the problem we are facing is the two-to-one map from $\bar{N}_{i}$ and $\bar{V}_{i}$ to $A_{i}$, and the map (3.44) contains one linear combination of $\bar{N}_{i}$ and $\bar{V}_{i}$, we will seek to find another independent linear combination of $\bar{N}_{i}$ and $\bar{V}_{i}$ - let us call it $\bar{X}^{i}$ - to integrate out. We want the equation of motion for $\bar{X}^{i}-$ let us call it $\bar{Y}_{i}=0$, where $\bar{Y}_{i} \sim \delta \overline{\mathcal{I}} / \delta \bar{X}^{i}$ up to a volume measure - to be diffeomorphism invariant. If $\bar{Y}_{i}$ transforms exactly like spatial components of a Lorentz 1-form, similar to that of $\bar{V}_{i}$ :

$$
\delta \bar{Y}_{i}=\bar{\xi}^{\mu} \partial_{\mu} \bar{Y}_{i}+\bar{Y}_{k} \partial_{i} \bar{\xi}^{k},
$$


then $\bar{Y}_{i}=0$ is diffeomorphism invariant. Since $\bar{Y}_{i}$ is a functional derivative with respect to $\bar{X}^{i}$, this requires $\bar{X}^{i}$ to transform exactly as spatial components of Lorentz vector $\bar{X}^{\mu}$ :

$$
\delta \bar{X}^{\mu}=\bar{\xi}^{\nu} \partial_{\nu} \bar{X}^{\mu}-\bar{X}^{\nu} \partial_{\nu} \bar{\xi}^{\mu} .
$$

From holography we have already had a good Lorentz 1-form $\bar{V}_{\mu}$, it is not hard to construct a Lorentz vector from it - just by raising the index $\mu$ with the full spacetime metric in ADM form. The result is

$$
\begin{aligned}
\bar{X}^{\mu} & =\left(-\frac{1}{\bar{N}^{2}}\left[\bar{V}_{t}-\bar{N}^{k} \bar{V}_{k}\right], \bar{G}^{i j} \bar{V}_{j}+\frac{1}{\bar{N}^{2}} \bar{N}^{i}\left[\bar{V}_{t}-\bar{N}^{j} \bar{V}_{j}\right]\right), \\
\bar{V}_{\mu} & =\left(\left[-\bar{N}^{2}+\bar{N}^{k} \bar{N}_{k}\right] \bar{X}^{t}+\bar{N}_{k} \bar{X}^{k}, \bar{N}_{i} \bar{X}^{t}+\bar{G}_{i j} \bar{X}^{j}\right)
\end{aligned}
$$

where the second line is the inverse of the first line. It is straightforward to check $\bar{X}^{\mu}$ defined in this way satisfies the above diffeomorphism transformation using (3.29)-(3.34). Then using the second line of the above equations, the condition $\delta \overline{\mathcal{I}} / \delta \bar{X}^{i}=0$ can be written explicitly as

$$
\bar{N}_{i} \frac{\delta \overline{\mathcal{I}}}{\delta \bar{V}_{t}}+\bar{G}_{i j} \frac{\delta \overline{\mathcal{I}}}{\delta \bar{V}_{j}}=0
$$

where the holographic on-shell action $\overline{\mathcal{I}}=\overline{\mathcal{I}}\left[\bar{V}_{\mu}, \bar{N}, \bar{N}_{i}, \bar{G}_{i j}, \bar{\phi}\right]$. This constraint equation means that we are integrating out $\bar{X}^{i}$ in $\overline{\mathcal{I}}$ while holding $\bar{X}^{t}, \bar{N}_{i}, \bar{N}, \bar{G}_{i j}$ and $\bar{\phi}$ fixed. It is diffeomorphism invariant as advertised, Weyl invariant (this is trivial, since its right hand side is zero) as well as gauge invariant (by analog to the definition of conserved current). In practice, it can be viewed as an equation for $\bar{N}_{i}$, which allows one to solve $\bar{N}_{i}$ (i.e. $v_{i}$ ) in terms of other fields. Thus it shall be viewed as part of the holographic dictionary. Together with (3.38)-(3.45), they completely determine the holographic map from the boundary fields of the bulk theory to the source fields of the non-relativistic field theory, allowing one to obtain a unique field theory effective action $\mathcal{W}$ from the holographic on-shell action $\overline{\mathcal{I}}$. In the next section, we will show that the effective action obtained this way is a good description of the quantum Hall effect, in agreement with previous results obtained from non-holographic approaches, and has a good LLL projection. From that we will further uncover the true physical interpretation of $v^{i}$ in the context of fractional quantum Hall effect.

\subsubsection{Choice (II): a field constraint}

(3.51) is not the only choice for constraint equation that is diffeomorphism and gauge invariant. Other than this type of constraint written in terms of functional derivatives of the action, which acquires a natural interpretation in term of path integral, we can write down another type of constraint using only the fields, without the action. It it straightforward to check that the following equation

$$
\bar{V}_{t i}+\bar{V}_{i j} \bar{G}^{j k} \bar{N}_{k}=0
$$

is also diffeomorphism, Weyl and gauge invariant. Here $\bar{V}_{\mu \nu}=\partial_{\mu} \bar{V}_{\nu}-\partial_{\nu} \bar{V}_{\mu}$. Even though the possible interpretation of this equation is different from (3.51)'s, it functions in the 
same way as the latter does: it is an equation for $\bar{N}_{i}$ and together with the rest of the holographic dictionary (3.38)-(3.45) completely and uniquely determines the field theory effective action $\mathcal{W}$ in terms of the sources $\Phi, g_{i j}$ and $A_{\mu}$. From holographic point of view, it can not be ruled out in favor of (3.51). Nor can it be from field theoretical considerations for quantum Hall problems, at least at the primitive level regarding only the universal transport properties, which we will discuss in detail in the next section. For example, it also has a good LLL projection. Actually, despite the seemingly different appearances of (3.51) and (3.52), for the quantum Hall effect at leading orders in derivative expansion where the action $\overline{\mathcal{I}}$ is dominated by gauge Chern-Simons term, the major part of the leading order of (3.51) and (3.52) are the same and the correlation functions they produce are also the same at leading orders. Thus unless one goes to a more detailed calculation involving higher order corrections or comes up with other physical considerations for the quantum Hall effect which favor one over the other, at the current level of discussion, we will view both constraints (3.51) and (3.52) are viable.

It is interesting to note that the left hand side of the above constraint (3.52) takes the same form as the combination appearing in the first term of the low energy gauge action (1.6) in Hořava-Lifshitz gravity theory. This part is roughly the energy density stored in the uniform electric field, and it depends on the shift function $\bar{N}_{i}$. The rest of the action is roughly minus of the energy density stored in the magnetic field, and is independent of $\bar{N}_{i}$. The former, the electric energy density, is a square of the "electric field", i.e. the left hand side of (3.52), thus is always non-negative. It only minimizes the action when it is zero, which implies the above constraint.

\section{Low energy Chern-Simons effective action of FQHE}

In this section we study a simple holographic model with non-dynamical Chern-Simons terms to show how the holographic dictionary we have just built can be used to derive an effective action for quantum Hall fluids, from which many universal properties inaccessible before can be extracted now. A similar holographic model with non-dynamical gauge Chern-Simons term is used in [98]. The infrared physics of quantum Hall effect at scale much larger than the magnetic length $1 / \sqrt{B}$ is dominated by the Chern-Simons term from the point of view of effective theory [5]. This give rise to many universal properties which are insensitive to local dynamics (at least at this scale). We will focus on extracting these universal properties at leading and, in some occasions, sub-leading orders, and they are always dominated by the Chern-Simons term. Terms in the effective action generated from the local dynamics - the contributions from non-Chern-Simons terms in effective theory or in holography, like those from (1.5) and (1.6) - are in general non-local. But for quantum Hall effects where $B$ field is large and system is gapped, these terms can be written as an series of derivative expansions and every term is local now. ${ }^{7}$ Almost every such term is sub-leading in the derivative expansion, except for one term which we will denote by

\footnotetext{
${ }^{7}$ In the following we will refer to this type of terms as "local terms", sometimes with a subscript "loc", on contrary to terms obtained from non-dynamical Chern-Simons terms, which are topological and non-local by nature.
} 
a general functional $f[B]$. The non-dynamical Chern-Simons terms in $3+1$ dimensional holography are essentially all boundary terms. This gives us several advantages. Since they are boundary terms, they are insensitive to the bulk dynamics which can not be uniquely determined from bottom-up approaches, but preserve the universality that we are mostly interested in. This allows us not to specify the local bulk dynamics, or specify it in a way as general as possible (for example, we assume only a general functional $f[B]$ later on to denote some possible leading order contributions from local terms without knowing or solving its explicit form). This grants maximum flexibility for later model-building when the universal properties we find here can still be valid. Last but not least, it simplifies the calculation and makes simple analytic results possible. More complicated models such as those involving dynamical Chern-Simons terms coupled to axion fields are beyond the scope of this paper and we will leave them to future research.

\subsection{Holographic gauge Chern-Simons term}

The bulk gauge Chern-Simons term $[157,158]$ is

$$
\hat{\mathcal{I}}_{\mathrm{CS}}=\frac{\nu}{16 \pi} \int d^{4} x \epsilon^{M N P Q} V_{M N} V_{P Q},
$$

where $V_{M N}=\partial_{M} V_{N}-\partial_{N} V_{M}$. For simplicity, we choose the coupling to be a constant $\nu$, rather than a dynamical axion. Then this term is essentially a boundary term:

$$
\overline{\mathcal{I}}_{\mathrm{CS}}=\frac{\nu}{4 \pi} \int_{r=0} d^{3} x \epsilon^{i j}\left\{\bar{V}_{t} \partial_{i} \bar{V}_{j}+\bar{V}_{i}\left(\partial_{j} \bar{V}_{t}-\partial_{t} \bar{V}_{j}\right)\right\}
$$

We denote the total on-shell action of the bulk theory to be

$$
\overline{\mathcal{I}}\left[\bar{V}_{\mu}, \bar{N}, \bar{N}_{i}, \bar{G}_{i j}, \bar{\phi}\right]=\overline{\mathcal{I}}_{\mathrm{CS}}\left[\bar{V}_{\mu}\right]+\overline{\mathcal{I}}_{\mathrm{loc}}\left[\bar{V}_{\mu}, \bar{N}, \bar{N}_{i}, \bar{G}_{i j}, \bar{\phi}\right]
$$

where $\overline{\mathcal{I}}_{\text {loc }}$ is the collection of all the other terms in the action, e.g. those involving local dynamics of the graviton and gauge field.

We will solve the model perturbatively by a derivative expansion. First, we adopt the power counting scheme commonly used in the context of quantum Hall effective theories (e.g. in [31]). Let $\epsilon$ be an infinitesimal book-keeping parameter that labels the orders of the derivative expansion (not to be confused with Levi-Civita symbol). We assume

$$
\partial_{i} \sim O(\epsilon), \quad \partial_{t} \sim O\left(\epsilon^{2}\right), \quad A_{i} \sim O\left(\epsilon^{-1}\right), \quad A_{t}, g_{i j}, \Phi \sim O(1) .
$$

Then $B \sim O(1), E_{i} \equiv \partial_{i} A_{t}-\partial_{t} A_{i} \sim O(\epsilon), \omega_{\mu} \sim O(\epsilon), \mathcal{R}^{(2)} \sim O\left(\epsilon^{2}\right)$. Thus according to the holographic dictionary, $\bar{V}_{i} \sim O\left(\epsilon^{-1}\right), \bar{V}_{t} \sim O(1), \bar{V}_{i j} \sim O(1), \bar{V}_{t i} \sim O(\epsilon)$. We further assume

$$
v_{i} \sim O(\epsilon), \quad \text { i.e. } \quad \bar{N}_{i} \sim O(\epsilon) .
$$

We will later need to check that this assumption is self-consistent. Even though at this time we do not know the specific form of $\overline{\mathcal{I}}_{\text {loc }}$, we know at least their basic structures allowed 
by symmetries. The leading orders in derivative expansion will have forms given in (1.5) and (1.6) [131]. This implies the following order estimation:

$$
\frac{\delta \overline{\mathcal{I}}_{\text {loc }}}{\delta \bar{V}_{i}} \sim O(\epsilon), \quad \frac{\delta \overline{\mathcal{I}}_{\text {loc }}}{\delta \bar{V}_{t}} \sim O\left(\epsilon^{2}\right), \quad \frac{\delta \overline{\mathcal{I}}_{\text {loc }}}{\delta \bar{N}_{i}} \sim O(\epsilon) .
$$

We further single out one particular term in $\overline{\mathcal{I}}_{\text {loc }}$, denoted by $f\left[\frac{1}{2} \bar{\varepsilon}^{i j} \bar{V}_{i j}\right]$ :

$$
\overline{\mathcal{I}}_{\text {loc }} \supset \int d^{3} x \bar{N} \sqrt{\bar{G}} f\left[\frac{1}{2} \bar{\varepsilon}^{i j} \bar{V}_{i j}\right]
$$

where $f$ is an arbitrary scalar functional of $\frac{1}{2} \bar{\varepsilon}^{i j} \bar{V}_{i j}$. The function $-f[B]$ is related to the energy density $\left\langle\mathcal{E}^{0}\right\rangle$ as a function of the magnetic field. ${ }^{8}$ This is the only term that contribute to $O(\epsilon)$ order from $\delta \overline{\mathcal{I}}_{\text {loc }} / \delta \bar{V}_{i}$ :

$$
\frac{\delta \overline{\mathcal{I}}_{\text {loc }}}{\delta \bar{V}_{i}}=\bar{N} f^{\prime \prime}\left[\frac{1}{2} \bar{\varepsilon}^{k^{\prime} l^{\prime}} \bar{V}_{k^{\prime} l^{\prime}}\right] \epsilon^{i j} \partial_{j}\left(\frac{1}{2} \bar{\varepsilon}^{k l} \bar{V}_{k l}\right)+f^{\prime}\left[\frac{1}{2} \bar{\varepsilon}^{k^{\prime} l^{\prime}} \bar{V}_{k^{\prime} l^{\prime}}\right] \epsilon^{i j} \partial_{j} \bar{N}+O\left(\epsilon^{2}\right) .
$$

Here one "'" is one derivative of $f$ with respect to its argument.

Using the holographic dictionary (3.38)-(3.45), the Chern-Simons term can be expanded as

$$
\begin{aligned}
\overline{\mathcal{I}}_{\mathrm{CS}}= & \int d^{3} x\left\{\frac{\nu}{4 \pi} \epsilon^{\rho \mu \nu} A_{\rho} \partial_{\mu} A_{\nu}-\frac{\nu}{2 \pi}\left(s+s^{\prime}\right) \epsilon^{\rho \mu \nu} \omega_{\rho} \partial_{\mu} A_{\nu}+\frac{\nu}{4 \pi}\left(s+s^{\prime}\right)^{2} \epsilon^{\rho \mu \nu} \omega_{\rho} \partial_{\mu} \omega_{\nu}\right\} \\
& +\frac{\nu}{4 \pi} \int d^{3} x \sqrt{g} e^{-\Phi}\left\{\left[m e^{2 \Phi}\left(-2 \varepsilon^{i j} E_{i} v_{j}-B v^{2}\right)+\left(s^{\prime}+1\right) e^{\Phi} E^{i} \partial_{i} \log \left(m e^{\Phi}\right)\right]\right. \\
& +\frac{(\mathrm{g}-2)}{2 m} B\left[B+\left(1-2 s-s^{\prime}\right) \mathcal{R}^{(2)}+\varepsilon^{i j} \omega_{i} \partial_{j}\left(s+s^{\prime}\right)\right. \\
& \left.-\varepsilon^{i j} \partial_{i}\left(\frac{s^{\prime}+1}{2} \varepsilon^{k l} g_{j k} \partial_{l} \log \left(m e^{\Phi}\right)\right)\right] \\
& \left.-\left(2-\frac{\mathrm{g}}{2}+s^{\prime}\right) e^{\Phi} B \varepsilon^{i j}\left[\partial_{i} v_{j}+v_{j} \partial_{i} \log \left(m e^{\Phi}\right)\right]\right\}+O\left(\epsilon^{3}\right) .
\end{aligned}
$$

By our power counting scheme, the third terms in the first line is of order $O\left(\epsilon^{4}\right)$, but since it takes the form of gravitational Chern-Simons form and its structure is unique compare to other possible terms at the same order, we write it down explicitly. (4.4) can be expanded as

$$
\begin{aligned}
\overline{\mathcal{I}}_{\mathrm{loc}} \supset & \int d^{3} x e^{-\Phi} \sqrt{g}\left\{f[B]+f^{\prime}[B]\left[-\varepsilon^{i j} \partial_{i}\left(\left(s+s^{\prime}\right) \omega_{j}\right)+\varepsilon^{i j} \partial_{i}\left(m e^{\Phi} v_{j}\right)\right.\right. \\
& \left.\left.+\nabla^{2}\left(\frac{s^{\prime}+1}{2} \log \left(m e^{\Phi}\right)\right)\right]+O\left(\epsilon^{3}\right)\right\} .
\end{aligned}
$$

\footnotetext{
${ }^{8}$ Our notation $f[B]$ emphasize that it has no dependence on mass $m$ and other source fields other than the metric-dependence through $B$. It can, however, depend on other couplings introduced through the interactions, such as the dimensionless $\lambda_{4 \mathrm{~F}}$ in (2.26) or other dimensionful couplings like that of the Coulomb interaction. For the 4-Fermi interaction which is scale invariant at $z=2$, just by dimensional analysis $f[B]$ must take the form
}

$$
f[B] \sim \lambda_{4 \mathrm{~F}} B^{2} .
$$




\subsection{Under the path integral constraint}

In this subsection, we use the path integral constraint (3.51) to calculate the effective action. Using (4.3) it can be written as

$$
-\frac{\nu}{2 \pi} \epsilon^{i j} \bar{V}_{t j}+\frac{\delta \overline{\mathcal{I}}_{\text {loc }}}{\delta \bar{V}_{i}}+\bar{N}^{i}\left(\frac{\nu}{4 \pi} \epsilon^{k l} \bar{V}_{k l}+\frac{\delta \overline{\mathcal{I}}_{\text {loc }}}{\delta \bar{V}_{t}}\right)=0 .
$$

At $O(\epsilon)$ order, this gives

$$
\begin{aligned}
\bar{N}^{i}= & \frac{\bar{\varepsilon}^{i j} \bar{V}_{t j}}{\frac{1}{2} \bar{\varepsilon}^{k l} \bar{V}_{k l}}-\frac{2 \pi}{\nu} \bar{N} f^{\prime \prime}\left[\frac{1}{2} \bar{\varepsilon}^{k^{\prime} l^{\prime}} \bar{V}_{k^{\prime} l^{\prime}}\right] \bar{\varepsilon}^{i j} \partial_{j} \log \left(\frac{1}{2} \bar{\varepsilon}^{k l} \bar{V}_{k l}\right) \\
& -\frac{2 \pi}{\nu} \frac{1}{\frac{1}{2} \bar{\varepsilon}^{k l} \bar{V}_{k l}} f^{\prime}\left[\frac{1}{2} \bar{\varepsilon}^{k^{\prime} l^{\prime}} \bar{V}_{k^{\prime} l^{\prime}}\right] \bar{\varepsilon}^{i j} \partial_{j} \bar{N}+O\left(\epsilon^{2}\right) .
\end{aligned}
$$

Applying the holographic dictionary, we can translate it into field theory language:

$$
\begin{aligned}
v^{i}= & \frac{\varepsilon^{i j} E_{j}}{B}+\frac{(\mathrm{g}-2) e^{-\Phi}}{4 m} \varepsilon^{i j} \partial_{j} \log \left(\frac{B}{m} e^{-\Phi}\right)+\frac{e^{-\Phi}}{4 m} \varepsilon^{i j} \partial_{j} \mathrm{~g} \\
& +\frac{2 \pi}{\nu} e^{-\Phi}\left(f^{\prime \prime}[B] \varepsilon^{i j} \partial_{j} \log B-\frac{1}{B} f^{\prime}[B] \bar{\varepsilon}^{i j} \partial_{j} \Phi\right)+O\left(\epsilon^{2}\right) .
\end{aligned}
$$

Plugging this into (4.6) and (4.7) we will get the final results for the effective action. The full expressions are very lengthy so we will not record it here. But when $\Phi=0$ and $m, \mathrm{~g}$, $s$ and $s^{\prime}$ are all constants, the expressions simplify to

$$
\begin{aligned}
\mathcal{W}_{\mathrm{CS}}= & \int d^{3} x\left\{\frac{\nu}{4 \pi} \epsilon^{\rho \mu \nu} A_{\rho} \partial_{\mu} A_{\nu}-\frac{\nu}{2 \pi}\left(s+s^{\prime}\right) \epsilon^{\rho \mu \nu} \omega_{\rho} \partial_{\mu} A_{\nu}+\frac{\nu}{4 \pi}\left(s+s^{\prime}\right)^{2} \epsilon^{\rho \mu \nu} \omega_{\rho} \partial_{\mu} \omega_{\nu}\right\} \\
& +\frac{\nu}{4 \pi} \int d t d^{2} \vec{x} \sqrt{g}\left\{m \frac{E^{2}}{B}-\left(2-\frac{\mathrm{g}}{2}+s^{\prime}\right) E^{i} \partial_{i} \log B+\frac{(\mathrm{g}-2)}{2 m} B\left[B+\left(1-2 s-s^{\prime}\right) \mathcal{R}^{(2)}\right]\right. \\
& \left.-\left[\frac{\mathrm{g}-2}{4}\left(\frac{3}{2}-\frac{\mathrm{g}}{4}+s^{\prime}\right) \frac{B}{m}+\left(1+s^{\prime}\right) \frac{2 \pi}{\nu} B f^{\prime \prime}[B]+\frac{m}{B}\left(\frac{2 \pi}{\nu} B f^{\prime \prime}[B]\right)^{2}\right]\left(\partial_{i} \log B\right)^{2}\right\} \\
& +O\left(\epsilon^{3}\right) .
\end{aligned}
$$

and

$$
\begin{aligned}
\mathcal{W}_{\text {loc }} \supset & \int d t d^{2} \vec{x} \sqrt{g}\left\{f[B]-\left(s+s^{\prime}\right) f^{\prime}[B] \mathcal{R}^{(2)}+m f^{\prime \prime}[B] E^{i} \partial_{i} \log B\right. \\
& \left.+\left(\frac{\mathrm{g}-2}{4} B f^{\prime \prime}[B]+\frac{2 \pi}{\nu} m B f^{\prime \prime}[B]^{2}\right)\left(\partial_{i} \log B\right)^{2}+O\left(\epsilon^{3}\right)\right\} .
\end{aligned}
$$

For simplicity, for now we assume the above terms are the only contributions to the effective action, i.e. $\mathcal{W}_{\text {loc }}$ contains only (4.4). It is not hard to compute additional terms if there are some, so we temporarily ignore them here. Then the total action takes the form

$$
\begin{aligned}
\mathcal{W}= & \int d^{3} x\left\{\frac{\nu}{4 \pi} \epsilon^{\rho \mu \nu} A_{\rho} \partial_{\mu} A_{\nu}-\frac{\nu}{2 \pi}\left(s+s^{\prime}\right) \epsilon^{\rho \mu \nu} A_{\rho} \partial_{\mu} \omega_{\nu}+\frac{\nu}{4 \pi}\left(s+s^{\prime}\right)^{2} \epsilon^{\rho \mu \nu} \omega_{\rho} \partial_{\mu} \omega_{\nu}\right\} \\
& +\int d^{3} x \sqrt{g}\left\{\alpha\left[B, E^{2}\right]+f_{0}\left[B, \mathcal{R}^{(2)}\right]+f_{1}[B] E^{i} \partial_{i} \log B+f_{2}[B]\left(\partial_{i} \log B\right)^{2}+O\left(\epsilon^{3}\right)\right\},
\end{aligned}
$$


where

$$
\begin{aligned}
\alpha & =\frac{\nu m E^{2}}{4 \pi B} \\
f_{0} & =f[B]+\frac{\nu(\mathrm{g}-2)}{8 \pi m} B\left[B+\left(1-2 s-s^{\prime}\right) \mathcal{R}^{(2)}\right]-\left(s+s^{\prime}\right) f^{\prime}[B] \mathcal{R}^{(2)}, \\
f_{1} & =-\frac{\nu}{2 \pi}\left(1-\frac{\mathrm{g}}{4}+\frac{s^{\prime}}{2}\right)+m f^{\prime \prime}[B] \\
f_{2} & =-\frac{\nu(\mathrm{g}-2)}{16 \pi}\left(\frac{3}{2}-\frac{\mathrm{g}}{4}+s^{\prime}\right) \frac{B}{m}-\left(1-\frac{\mathrm{g}}{4}+\frac{s^{\prime}}{2}\right) B f^{\prime \prime}[B]+\frac{\pi m}{\nu} B f^{\prime \prime}[B]^{2} .
\end{aligned}
$$

The three terms in the first line can be combined into a single "complete square" term of $A-\left(s+s^{\prime}\right) \omega$, the same structure appearing in $[24,67]$.

\subsection{Correlation functions of electromagnetic response}

Using formulae given in appendix A, it is straightforward to compute the correlation functions. The 1-point functions of the conserved current is

$$
\begin{aligned}
\left\langle J^{t}\right\rangle= & \frac{\nu}{2 \pi}\left[B-\left(s+s^{\prime}\right) \mathcal{R}^{(2)}-m \nabla_{i}\left(\frac{E^{i}}{B}\right)\right]-\nabla_{i}\left(f_{1} \nabla^{i} \log B\right)+O\left(\epsilon^{3}\right) \\
\left\langle J^{i}\right\rangle= & \frac{\nu}{2 \pi} \varepsilon^{i j}\left[E_{j}-\left(s+s^{\prime}\right)\left(\partial_{j} \omega_{t}-\partial_{t} \omega_{j}\right)\right]+\frac{1}{\sqrt{g}} \partial_{t}\left[\sqrt{g}\left(\frac{\nu m E^{i}}{2 \pi B}+f_{1} \nabla^{i} \log B\right)\right] \\
& +\varepsilon^{i j} \nabla_{j}\left[-\frac{\nu m E^{2}}{4 \pi B^{2}}+f_{0}^{\prime}-\frac{f_{1}}{B} \nabla_{k} E^{k}-f_{2}^{\prime}\left(\nabla_{k} \log B\right)^{2}-2 \frac{f_{2}}{B} \nabla^{2} \log B\right]+O\left(\epsilon^{4}\right) .
\end{aligned}
$$

For flat background with constant $B$ and $E_{i}$ fields, the 2-point functions have the following structures governed by Ward Identities

$$
\begin{aligned}
\left\langle J^{t}(x) J^{t}(0)\right\rangle= & i \Pi_{0} \vec{\partial}^{2} \delta(x), \\
\left\langle J^{t}(x) J^{i}(0)\right\rangle= & -i \Pi_{0} \partial^{i} \partial_{t} \delta(x)+i \Pi_{1} \epsilon^{i j} \partial_{j} \delta(x)-i \Pi_{3} \epsilon^{i j} E^{k} \partial_{k} \partial_{j} \delta(x), \\
\left\langle J^{i}(x) J^{t}(0)\right\rangle= & -i \Pi_{0} \partial^{i} \partial_{t} \delta(x)-i \Pi_{1} \epsilon^{i j} \partial_{j} \delta(x)-i \Pi_{3} \epsilon^{i j} E^{k} \partial_{k} \partial_{j} \delta(x), \\
\left\langle J^{i}(x) J^{j}(0)\right\rangle= & i \Pi_{0} \delta^{i j} \partial_{t}^{2} \delta(x)+i \Pi_{1} \epsilon^{i j} \partial_{t} \delta(x)+i \Pi_{2}\left(\delta^{i j} \vec{\partial}^{2}-\partial^{i} \partial^{j}\right) \delta(x) \\
& +i \Pi_{3}\left(\epsilon^{j k} E^{i}+\epsilon^{i k} E^{j}\right) \partial_{k} \partial_{t} \delta(x)
\end{aligned}
$$

with

$$
\begin{aligned}
\Pi_{0}= & \frac{\nu m}{2 \pi B}+O(\epsilon) \\
\Pi_{1}= & \frac{\nu}{2 \pi}+\frac{\nu}{2 \pi B}\left[\left(1-\frac{\mathrm{g}}{4}+\frac{s^{\prime}}{2}\right)-\frac{2 \pi m}{\nu} f^{\prime \prime}[B]\right] \vec{\partial}^{2}+O\left(\epsilon^{3}\right) \\
\Pi_{2}= & f^{\prime \prime}[B]+\frac{\nu(\mathrm{g}-2)}{4 \pi m} \\
& +\left\{\frac{\nu(\mathrm{g}-2)}{4 \pi m B}\left(\frac{3}{4}-\frac{\mathrm{g}}{8}+\frac{s^{\prime}}{2}\right)+2 \frac{f^{\prime \prime}[B]}{B}\left[\left(1-\frac{\mathrm{g}}{4}+\frac{s^{\prime}}{2}\right)-\frac{\pi m}{\nu} f^{\prime \prime}[B]\right]\right\} \vec{\partial}^{2} \\
& +\frac{\nu m E^{2}}{2 \pi B^{3}}+O\left(\epsilon^{3}\right) \\
\Pi_{3}= & -\frac{\nu m}{2 \pi B^{2}}+O(\epsilon) .
\end{aligned}
$$


Here $\vec{\partial}^{2} \equiv \delta^{i j} \partial_{i} \partial_{j}$. These are the same as those derived in [61]. ${ }^{9}$ Notice that $\Pi_{1}$ is actually the Hall conductivity $\sigma_{H}$ defined in the usual way. Here we largely ignore the response to curvature by setting the background to be flat, but they can also be calculated explicitly and compared with the literature, for example [68, 159].

\subsection{Under the field constraint}

In parallel to the previous subsection, in this subsection we switch to the field constraint (3.52). It can be solved as

$$
\bar{N}^{i}=\frac{\bar{\varepsilon}^{i j} \bar{V}_{t j}}{\frac{1}{2} \bar{\varepsilon}^{k l} \bar{V}_{k l}} .
$$

Applying the holographic dictionary, we can translate it into field theory language as

$$
v^{i}=\frac{\varepsilon^{i j} E_{j}}{B}+\frac{(\mathrm{g}-2) e^{-\Phi}}{4 m} \varepsilon^{i j} \partial_{j} \log \left(\frac{B}{m} e^{-\Phi}\right)+\frac{e^{-\Phi}}{4 m} \varepsilon^{i j} \partial_{j} g+O\left(\epsilon^{2}\right) .
$$

Again plugging this into (4.6) and (4.7) we will get lengthy expressions for the effective action. When $\Phi=0$ and $m, \mathrm{~g}, s$ and $s^{\prime}$ are all constants, the results are

$$
\begin{aligned}
\mathcal{W}_{\mathrm{CS}}= & \int d^{3} x\left\{\frac{\nu}{4 \pi} \epsilon^{\rho \mu \nu} A_{\rho} \partial_{\mu} A_{\nu}-\frac{\nu}{2 \pi}\left(s+s^{\prime}\right) \epsilon^{\rho \mu \nu} \omega_{\rho} \partial_{\mu} A_{\nu}+\frac{\nu}{4 \pi}\left(s+s^{\prime}\right)^{2} \epsilon^{\rho \mu \nu} \omega_{\rho} \partial_{\mu} \omega_{\nu}\right\} \\
& +\frac{\nu}{4 \pi} \int d^{3} x \sqrt{g}\left\{m \frac{E^{2}}{B}-\left(2-\frac{\mathrm{g}}{2}+s^{\prime}\right) E^{i} \partial_{i} \log B+\frac{(\mathrm{g}-2)}{2 m} B\left[B+\left(1-2 s-s^{\prime}\right) \mathcal{R}^{(2)}\right]\right. \\
& \left.-\left[\frac{\mathrm{g}-2}{4}\left(\frac{3}{2}-\frac{\mathrm{g}}{4}+s^{\prime}\right) \frac{B}{m}\right]\left(\partial_{i} \log B\right)^{2}\right\}+O\left(\epsilon^{3}\right) .
\end{aligned}
$$

and

$$
\begin{aligned}
\mathcal{W}_{\text {loc }} \supset & \int d^{3} x \sqrt{g}\left\{f[B]-\left(s+s^{\prime}\right) f^{\prime}[B] \mathcal{R}^{(2)}+m f^{\prime \prime}[B] E^{i} \partial_{i} \log B\right. \\
& \left.+\frac{\mathrm{g}-2}{4} B f^{\prime \prime}[B]\left(\partial_{i} \log B\right)^{2}+O\left(\epsilon^{3}\right)\right\} .
\end{aligned}
$$

Comparing with the corresponding results in the previous subsection using path integral constraint (3.51), we see the only difference is that contributions from the local terms of the action (those involving $f[B]$ and its derivatives) are absent in the constraint equation, and consequently in the expressions of $v^{i}$ and Chern-Simons action. The field constraint (3.52) is equivalent to the path integral constraint (3.51) when only the Chern-Simons action is considered in the latter. This is the reason that for the quantum Hall effect, these two constraints are equivalent at the leading order when magnetic field is big and ChernSimons term dominates. If one considers other problems where Chern-Simons term is not important, these two constraints will give quite different results. Then one has to weigh which constraint is more appropriate under other physical considerations.

\footnotetext{
${ }^{9}$ To compare with [61], we need $s^{\prime} \Rightarrow s, E_{i} \Rightarrow 0, f[B] \Rightarrow-\epsilon[\rho]$ with $\frac{\nu}{2 \pi} B \Rightarrow \rho$, where on the right hand side of each " $\Rightarrow$ " are the notations of [61].
} 
Now same as in the previous subsection, let us assume these are the only contributions to the effective action. The effective action still takes the same form as in (4.12), so do the functions $\alpha\left[B, E^{2}\right], f_{0}\left[B, \mathcal{R}^{(2)}\right]$ and $f_{1}[B]$. The only difference is the form of $f_{2}[B]$, which is now

$$
f_{2}=-\frac{\nu(\mathrm{g}-2)}{16 \pi}\left(\frac{3}{2}-\frac{\mathrm{g}}{4}+s^{\prime}\right) \frac{B}{m}+\frac{\mathrm{g}-2}{4} B f^{\prime \prime}[B] .
$$

For 1-point functions, $\left\langle J^{t}\right\rangle$ remains the same as before, so do most of the 2-point functions in (4.19)-(4.22). The only differences are $O\left(\epsilon^{3}\right)$ order of $\left\langle J^{i}\right\rangle$ and $O\left(\epsilon^{2}\right)$ order of $\Pi_{2}$ in $\left\langle J^{i}(x) J^{j}(0)\right\rangle$, which is now

$$
\begin{aligned}
\Pi_{2}= & f^{\prime \prime}[B]+\frac{\nu(\mathrm{g}-2)}{4 \pi m}+\left[\frac{\nu(\mathrm{g}-2)}{4 \pi m B}\left(\frac{3}{4}-\frac{\mathrm{g}}{8}+\frac{s^{\prime}}{2}\right)-\left(\frac{\mathrm{g}-2}{2}\right) \frac{f^{\prime \prime}[B]}{B}\right] \vec{\partial}^{2} \\
& +\frac{\nu m E^{2}}{2 \pi B^{3}}+O\left(\epsilon^{3}\right) .
\end{aligned}
$$

The functional expression of $\left\langle J^{i}\right\rangle$ in terms of $f_{2}$ and others are the same as (4.18).

To the orders of perturbative expansion we have computed, the two different constraints, the path integral one (3.51) and the field one (3.52) yield different results only at $O\left(m^{0}\right)$ and higher order of $m$ for $f_{2}$, thus for $\left\langle J^{i}\right\rangle$ and $\Pi_{2}$ at the corresponding orders. However, these differences are non-universal parts of the correlation functions as discussed in [61]. For the universal parts, for example, the $O\left(\mathrm{~m}^{-1}\right)$ order terms, the two constraints always produce the same results. Thus at the level of our current discussion, we do not favor one constraint over the other. We view both of them are viable. One of them may be a better choice when a specific problem is studied using a more detailed model, but we will leave this to the future.

\subsection{Holographic gravitational Chern-Simons term for $z>1$}

We can also add the gravitational Chern-Simons term [160] to our holographic model:

$$
\hat{\mathcal{I}}_{\mathrm{PY}}=\frac{c_{g}}{384 \pi} \int d^{4} x \epsilon^{P Q R S} R_{R S}^{M N} R_{N M P Q},
$$

where $R_{M N P Q}$ is the Riemann tensor constructed from the full 4-dimensional spacetime metric and indices $M, N, P, Q, R$ and $S$ run through $t, x, y$ and $r$. The subscript "PY" stands for "Pontryagin". Here for simplicity, we also assume the coupling is a constant $c_{g}$, rather than a dynamical axion scalar. Then it is a boundary term as well:

$$
\overline{\mathcal{I}}_{\mathrm{PY}}=\frac{c_{g}}{96 \pi} \int_{r=0} d^{3} x \sqrt{\bar{G}} \bar{\varepsilon}^{i j} \overline{\mathcal{P}}_{i j},
$$

where $\bar{\varepsilon}^{i j} \overline{\mathcal{P}}_{i j}$ is computed explicitly in appendix C. Unlike the boundary form of the gauge Chern-Simons term, which involves only $\partial_{t}$ and $\partial_{i}$ derivatives, $\overline{\mathcal{P}}_{i j}$ contains $\partial_{r}$ derivative terms as well. However, all and only these $\partial_{r}$ terms will be removed by adding a GibbonsHawking type boundary term [161]:

$$
\overline{\mathcal{I}}_{\partial \mathrm{PY}}=\frac{c_{g}}{48 \pi} \int_{r=0} d^{3} x \sqrt{-\gamma}\left(n_{M} \varepsilon^{M N P Q} \mathcal{K}_{N}{ }^{R} \nabla_{P} \mathcal{K}_{Q R}\right)
$$


where $\gamma_{M N}$ is the induced full spacetime metric at the boundary, $n_{M}$ the out-going unit normal vector of the boundary and $\mathcal{K}_{M N}=\gamma_{M}{ }^{P} \gamma_{N}{ }^{Q} \nabla_{P} n_{Q}$ the extrinsic curvature of the boundary. This term is added such that the Dirichlet boundary value problem is well posed, in a similar fashion as Gibbons-Hawking term is added to the Einstein-Hilbert action. Under the gauge conditions (3.11)-(3.13), $\mathcal{K}_{M N}=-\frac{L}{r} \Gamma_{M N}^{r}$ contains only $\partial_{r}$ derivative terms, thus it will only remove $\partial_{r}$ terms from the Chern-Simons action, and it is straightforward to check that it does remove all such terms.

From now on for the discussion of gravitational Chern-Simons term we assume

$$
z>1 \text {. }
$$

In appendix $\mathrm{C}$ we have computed

$$
\begin{aligned}
\bar{\varepsilon}^{i j} \overline{\mathcal{P}}_{i j}= & -2 \bar{\varepsilon}^{i j}\left[\omega_{t} \partial_{i} \omega_{j}+\omega_{i}\left(\partial_{j} \omega_{t}-\partial_{t} \omega_{j}\right)\right]+\bar{\varepsilon}^{i j}\left[\left(\bar{\varepsilon}^{k l} \partial_{k} \bar{N}_{l}\right) \partial_{i} \omega_{j}+\omega_{i} \partial_{j}\left(\bar{\varepsilon}^{k l} \partial_{k} \bar{N}_{l}\right)\right] \\
& +2 \bar{\varepsilon}^{i j}\left[\left(\nabla_{j} \nabla_{k} \bar{N}\right) \bar{K}_{i}^{k}-\left(\nabla_{k} \bar{N}\right) \nabla_{j} \bar{K}_{i}^{k}\right]+O\left(\partial_{r}\right)
\end{aligned}
$$

where

$$
\bar{K}_{i j}=\frac{1}{2 \bar{N}}\left(\partial_{t} \bar{G}_{i j}-\nabla_{i} \bar{N}_{j}-\nabla_{j} \bar{N}_{i}\right)
$$

is the extrinsic curvature of the global time foliation at the boundary. Then the gravitational Chern-Simons term, including the contribution from the boundary term (4.34), is

$$
\begin{aligned}
\overline{\mathcal{I}}_{\mathrm{PY}}= & -\frac{c_{g}}{48 \pi} \int d^{3} x \sqrt{\bar{G}} \bar{\varepsilon}^{i j}\left[\omega_{t} \partial_{i} \omega_{j}+\omega_{i}\left(\partial_{j} \omega_{t}-\partial_{t} \omega_{j}\right)\right] \\
& +\frac{c_{g}}{24 \pi} \int d^{3} x \sqrt{\bar{G}}\left\{\bar{\varepsilon}^{i j} \bar{K}_{i k}\left(\nabla_{j} \nabla^{k} \bar{N}\right)+\frac{1}{2} \mathcal{R}^{(2)}\left(\bar{\varepsilon}^{i j} \partial_{i} \bar{N}_{j}\right)\right\} .
\end{aligned}
$$

Applying the holographic map, we have

$\mathcal{W}_{\mathrm{PY}}=-\frac{c_{g}}{48 \pi} \int d^{3} x \epsilon^{\rho \mu \nu} \omega_{\rho} \partial_{\mu} \omega_{\nu}+\frac{c_{g}}{24 \pi} \int d^{3} x \sqrt{g}\left\{\varepsilon^{i j} K_{i k}\left(\nabla_{j} \nabla^{k} e^{-\Phi}\right)-\frac{1}{2} \mathcal{R}^{(2)}\left(\varepsilon^{i j} \partial_{i} v_{j}\right)\right\}$,

where

$$
K_{i j}=\frac{1}{2} e^{\Phi}\left(\partial_{t} g_{i j}+\nabla_{i} v_{j}+\nabla_{j} v_{i}\right)
$$

This term (4.35) shall now be added to the total effective action (4.3). By our power counting scheme, every term in (4.35) is of order $O\left(\epsilon^{4}\right)$. The gravitational Chern-Simons term only gives subleading order contributions to $\left\langle J^{\mu}\right\rangle$ when the spacetime is not flat, and does not alter the flat-spacetime current 2-point functions we compute earlier, but they will give non-trivial contributions to energy-stress correlation functions.

The gravitational Chern-Simons term describes the thermal Hall (Leduc-Righi) effect [162] and other parity-violating thermal transport phenomena [63, 64, 163]. The thermal Hall conductivity is proportional to the coefficient $c_{g}$, which in turn is related to the gravitational anomaly and corresponds to the central charge of the chiral CFT on the boundary [164-168]. The first term of (4.35) has appeared in the effective action of [67,68] and the whole action appeared in a different form written in Lorentzian notations with Christoffel symbol in [73], in addition to some of the aforementioned references. In the rest of this paper, we will not go further in this direction to pursue the thermal Hall effect. 


\section{Gravitational response and geometric properties of FQHE}

In this section, we will use the effective actions and correlation functions derived in the last section to analyze geometric properties and gravitational response of the fractional quantum Hall fluids they describe, focusing on Hall viscosity, angular momentum density, momentum density and their relations. The following discussions on viscosities and angular momentum density are carried out only for constant $B$. The results do not depend on $f_{2}$, thus the choice of the two constraints (3.51) or (3.52) does not matter in this section. We will work in flat space with $g_{i j}=\delta_{i j}, \Phi=0$ and $m, \mathrm{~g}, s$ and $s^{\prime}$ are all constants, unless otherwise stated.

\subsection{Wen-Zee shift and Hall viscosity}

The first line of the effective action (4.12) includes the three well known topological terms which do not depend on metric, and is in agreement with [24] and many subsequent works. Of particular interest to us is the second term $\omega \wedge \mathrm{d} A$, the so-called Wen-Zee term, whose coefficient we can identify as the shift $\mathcal{S}$ :

$$
\mathcal{S}=-2\left(s+s^{\prime}\right) .
$$

Thus the physical meaning of the constant $s^{\prime}$ arising from our holographic dictionary (3.43) and (3.44) is clear: it is related to the shift $\mathcal{S}$ by the above equation, while $s$ is the intrinsic spin of the microscopic field $\psi$ in the action (2.7). A similar relation and the relation between the shift and the conformal field theory construction [169] of fractional quantum Hall effect can be found in $[29,30]$. The shift is the offset between the total charge $Q=\int d^{2} \vec{x} \sqrt{g}\left\langle J^{t}\right\rangle$ and the total magnetic flux $N_{\phi}=\frac{1}{2 \pi} \int d^{2} \vec{x} \sqrt{g} B$ if the manifold where the quantum Hall fluid lives on has a non-trivial topology [24, 61, 170]:

$$
Q=\nu\left(N_{\phi}+\frac{\mathcal{S}}{2} \chi\right)
$$

where $\chi=\frac{1}{2 \pi} \int d^{2} \vec{x} \sqrt{g} \mathcal{R}^{(2)}$ is the Euler characteristic of the spatial manifold.

Another important aspect of the shift is that even the manifold's topology is trivial and the system lives in flat space, it is still related to Hall viscosity [29-31, 61]. Hall viscosity is one of the parity-odd dissipationless first order transport coefficients appearing in $(2+1)$ dimensional parity violating hydrodynamics [171-175]. Using $\omega_{t}=\frac{1}{2} \delta^{i j} \epsilon^{k l}\left(\delta g_{i k}\right) \partial_{t}\left(\delta g_{j l}\right)$ and $\mathcal{W}=2 \int d^{3} x \eta_{H} \omega_{t}+\ldots$, the Hall viscosity $\eta_{H}$ can be read off from the $\omega \wedge \mathrm{d} A$ term in the effective action:

$$
\eta_{H}=-\frac{\nu}{4 \pi}\left(s+s^{\prime}\right) B=\frac{1}{4} \mathcal{S}\left\langle J^{t}\right\rangle .
$$

Here we have assumed flat space with constant $B$ field and vanishing $E_{i}$ field.

\subsection{Magnetization and momentum density}

From (4.18), we can identify the long expression inside $\varepsilon^{i j} \nabla_{j}[\ldots]$ as the magnetization defined in [176]:

$$
\langle M\rangle=f_{0}^{\prime}-\frac{\nu m E^{2}}{4 \pi B^{2}}-\frac{f_{1}}{B} \nabla_{k} E^{k}-f_{2}^{\prime}\left(\nabla_{k} \log B\right)^{2}-2 \frac{f_{2}}{B} \nabla^{2} \log B .
$$


For constant $B$ and $E_{i}=0$, this reduces to

$$
\langle M\rangle=f_{0}^{\prime}=f^{\prime}[B]+\frac{\nu(\mathrm{g}-2)}{4 \pi m} B .
$$

The first half of the above result $\langle M\rangle=f_{0}^{\prime}$ is in agreement with the recent result of [73]. ${ }^{10}$ Using (4.17), (4.18) and (2.37) we can compute the momentum density

$$
\begin{aligned}
\left\langle p^{i}\right\rangle= & \frac{\nu m}{2 \pi} \varepsilon^{i j} E_{j}+\partial_{t}\left(\frac{\nu m^{2} E^{i}}{2 \pi B}+m f_{1} \partial^{i} \log B\right)+\varepsilon^{i j} \partial_{j}\left[m f_{0}^{\prime}-\frac{\mathrm{g}-2 s}{4} \frac{\nu}{2 \pi} B\right. \\
& -\frac{\nu m^{2} E^{2}}{4 \pi B^{2}}-\frac{\mathrm{g}-2 s}{4} \frac{\nu m}{2 \pi B} E^{k} \partial_{k} \log B+\left(\frac{\mathrm{g}-2 s}{4} \frac{\nu}{2 \pi}-f_{1}\right) \frac{m}{B} \partial_{k} E^{k} \\
& \left.+\left(\frac{\mathrm{g}-2 s}{4} B f_{1}^{\prime}-m f_{2}^{\prime}\right)\left(\partial_{k} \log B\right)^{2}+\left(\frac{\mathrm{g}-2 s}{4} f_{1}-2 \frac{m f_{2}}{B}\right) \partial_{k}^{2} \log B\right]+O\left(\epsilon^{4}\right) .
\end{aligned}
$$

The total angular momentum $\left\langle L_{\text {tot }}\right\rangle$ and its density $\ell_{\text {tot }}(x)$ are defined as

$$
\left\langle L_{\text {tot }}\right\rangle=\int d^{2} \vec{x} \epsilon_{i j} x^{i}\left\langle p^{j}(x)\right\rangle=\int d^{2} \vec{x} \ell_{\text {tot }}(x)+\ldots
$$

where the ... denotes the contribution to total angular momentum that is not translational invariant, thus can not be attribute to angular momentum density. In the above expression of $\left\langle p^{i}\right\rangle$, the quantity inside $\varepsilon^{i j} \nabla_{j}[\ldots]$ is half of the total angular momentum density $\ell_{\text {tot }}$, as can be seen by plugging the expression into the definition of angular momentum and integrating by parts. For constant $B$ field and $E_{i}=0$, the total angular momentum density is

$$
\ell_{\mathrm{tot}}=2\left(m f_{0}^{\prime}-\frac{\mathrm{g}-2 s}{4} \frac{\nu}{2 \pi} B\right)=-\frac{\nu}{\pi}\left(1-\frac{\mathrm{g}}{4}-\frac{s}{2}\right) B+2 m f^{\prime}[B] .
$$

Its relation to magnetization is

$$
\ell_{\text {tot }}=2 m\langle M\rangle+\left(s-\frac{\mathrm{g}}{2}\right)\left\langle J^{t}\right\rangle .
$$

\subsection{Vorticity and guiding center angular momentum density}

Next, let us look at the vorticity. From (4.9) and (4.27) we can see that $v^{i}$ has a form of the drift velocity $\left(\varepsilon^{i j} E_{j} / B\right.$, plus corrections for inhomogeneous $B$ field). Having the velocity, the vorticity associated with this motion is usually defined as curl of the velocity:

$$
\Omega=\varepsilon^{i j} \partial_{i} v_{j}
$$

Using (4.9) or (4.27) this can be rewritten as $\Omega=-\nabla_{i}\left(E^{i} / B\right)+\ldots$. Thus the vorticity is proportional to divergence of electric field in a constant magnetic field. One possible source of this divergence could be the impurity, i.e. $\nabla_{i} E^{i}$ equals to the density of impurity $\rho_{\text {imp }}$. Such identification has been made, for example, in [177]. Integrating by parts the $f_{1}[B]$ term in (4.12), this term can be written as

$$
\mathcal{W}=\int d^{3} x \sqrt{g}\left\{\left(\int f_{1}[B] d B\right) \Omega+\ldots\right\} .
$$

\footnotetext{
${ }^{10}$ Our $f_{0}$ plays the role of $f$ in [73], which is minus of the unperturbed energy density.
} 
This is the effective action $\mathcal{W}$ 's dependence on $\Omega$. We can see that in the effective action, terms involving divergence of the the electric field $\nabla_{i} E^{i}$ are related to vorticity. In holography, the Euclidean on-shell action $\overline{\mathcal{I}}_{\text {Eucl }}$ with $t$ integrated from 0 to $1 / T$ is $-T$ multiplying the grand potential $\Omega_{\text {grand }}$ (not to confused with the vorticity $\Omega$ ). Here $T$ is temperature and $\Omega_{\text {grand }}=-T \log \mathcal{Z}_{\text {grand }}$ where $\mathcal{Z}_{\text {grand }}$ is the partition function of the grand canonical ensemble. After going through this procedure, we see that the integrand (Lagrangian density) of $\mathcal{W}$ is the thermodynamical pressure $P=-\delta \Omega_{\text {grand }} / \delta V$ where $V=\int d^{2} \vec{x} \sqrt{g}$ is the volume. The same identification is used in [178] to study the classical Hall effect. [171] has given the relation between the partition function, angular momentum $L_{\text {orb }}$ and vorticity: $\mathcal{Z}_{\text {grand }}=\exp \left(L_{\mathrm{gc}} \Omega / 2 T+\ldots\right)$, then we have

$$
P=\frac{1}{2} \ell_{\mathrm{gc}} \Omega+\ldots, \quad \text { where } \quad \mathcal{W}=\int d^{3} x \sqrt{g} P .
$$

A similar relation has been derived in [49]. Now we have the angular momentum density conjugate to vorticity:

$$
\ell_{\mathrm{gc}}=2 \int f_{1}[B] d B=-\frac{\nu}{2 \pi}\left(2-\frac{\mathrm{g}}{2}+s^{\prime}\right) B+2 m f^{\prime}[B] .
$$

Notice that we have added a subscript "gc" (stands for "guiding center", whose meaning will be explained later) for $L_{\mathrm{gc}}$ and $\ell_{\mathrm{gc}}$ to distinguish them from the total angular momentum $L_{\text {tot }}$ and its density $\ell_{\text {tot }}$ discussed earlier. The explicit expressions of $\ell_{\text {tot }}$ and $\ell_{\text {gc }}$ also show that they are different. In [49] it has been shown that $L_{\text {tot }}$ defined from $\left\langle p^{i}\right\rangle$ is the corrected total kinetic angular momentum, and the authors also notice that the quantity $2 \delta P / \delta \Omega$ as what we call $\ell_{\mathrm{gc}}$ here does not have a direct interpretation as the total angular momentum density $\ell_{\text {tot }}$. To keep our focus on the derivations, we will postpone the explanation of the physical meaning of this so-called guiding center angular momentum density in the context of the quantum Hall effect to the end of the section. At this moment, from theoretical mechanics's point of view, vorticity $\Omega$ can be viewed as a temporal derivative of some generalized coordinate (some angle) and $\ell_{\mathrm{gc}}$ is the generalized momentum conjugate to it, which does not equal to the total angular momentum density. In terms of magnetization and charge density, $\ell_{\mathrm{gc}}$ can be expressed as

$$
\ell_{\mathrm{gc}}=2 m\langle M\rangle+\left(-s^{\prime}-\frac{\mathrm{g}}{2}\right)\left\langle J^{t}\right\rangle .
$$

It is worthy to note that the identification of $\Omega=\varepsilon^{i j} \partial_{i} v_{j}$ and $\ell_{\mathrm{gc}}=2 \int f_{1}[B] d B$ does not depend on the explicit expression (4.9) or (4.27). It can be directly made from (4.6) and (4.7), thus depends only on the holographic dictionary (3.38)-(3.45). Hence our results for guiding center angular momentum densities equally apply to the case when $\bar{N}_{i}$ is constrained by the bulk dynamics to be a functional of other boundary fields and $v^{i}$ takes some other form other than the drift velocity given in (4.9) or (4.27).

\subsection{Internal angular momentum density}

Since the guiding center angular momentum density is different from the total angular momentum density, we can define their difference as the internal angular momentum density: 
$\ell_{\text {int }}=\ell_{\text {tot }}-\ell_{\text {gc }}$. Thus we have

$$
\ell_{\text {int }}=\frac{\nu}{2 \pi}\left(s+s^{\prime}\right) B=-\frac{\mathcal{S}}{2}\left\langle J^{t}\right\rangle,
$$

where the subscript "int" stands for "internal". This agrees with the results found in [29, 30], where $-\mathcal{S} / 2$ is interpreted as conformal spin or mean orbital spin per particle. Here to clarify some potential confusion about terminology, we note that the quantity $\ell_{\text {int }}$ has different names in the literature. In [29] it is called "conformal spin density", in [24, 30] "mean orbital spin density", in [47] "intrinsic angular momentum density" and recently in [52] related to "Landau orbit spin". We will call it "internal angular momentum density" and avoid using the terms "intrinsic" and "spin" so as not to confuse with the intrinsic spin of particles in the usual sense in high energy physics. In this paper, the quantity $s$ will be called the intrinsic spin of the microscopic field $\psi$, which can be either a fundamental particle like the electron or a composite particle like composite boson or fermion. For example, if $\psi$ denotes an Dirac electron in vacuum, $s=1 / 2$. For Laughlin states with filling factor $\nu=1 /(2 n+1)$ where $n \in \mathbb{N}, \mathcal{S}=2 n+1$ [24]. Then the mean internal angular momentum per particle $-\mathcal{S} / 2=-n-1 / 2$. [151] derives the same result using both composite fermion and boson theories. Comparing our action (2.7) with those in [151], $\psi$ can denote composite fermions with intrinsic $\operatorname{spin}^{11} s=-n$ for the former theory and composite bosons with $s=-n-1 / 2$ for the latter. Hence the parameter $s^{\prime}$ arising from the map (3.43) and (3.44) takes value $s^{\prime}=-1 / 2$ for composite fermions and $s^{\prime}=0$ for composite bosons. The former corresponds to orbital spin of composite fermions at filling factor $\nu=1$. The intrinsic spin $s$ and the orbital spin $s^{\prime}$ of the composite particles together give the total internal angular momentum given by (5.15).

Now we can immediately recognize the simple relation between Hall viscosity and the internal angular momentum density:

$$
\eta_{H}=-\frac{1}{2} \ell_{\text {int }} .
$$

This is the well-known relation of [30, 47, 48]. The key point of reproducing this relation is to correctly identify the internal angular momentum density. Only the internal part satisfies this relation, as pointed out in [30, 47, 48], but not the total angular momentum density, unless the guiding center part vanishes. ${ }^{12,13}$ The Chern-Simons model generally has non-vanishing guiding center angular momentum density, as is shown here. So it is crucial

\footnotetext{
${ }^{11}$ The quantity we call $s$ here is called topological spin in [151]. We also notice there are different sign conventions in the literature, thus some of our expressions like those for spins have different signs compared with other references. This is due to various normalization choices for the charge, spin, Hall viscosity, alignment of the angular momentum, or combinations of them. For example, in [151] the charge is set to 1 while we set to -1 , hence our conventions for the intrinsic (topological) spin $s$ will also differ by a sign. They have $s=n$ and $n+1 / 2$ for composite fermions and bosons, respectively. Equivalently, the sign of Wen-Zee shift can be flipped.

$12[47]$ ensures this by assuming total angular momentum density is proportional to particle density $\left(\left\langle J^{t}\right\rangle\right.$ here), which could be in general violated by $f^{\prime}[B]$ term in $\ell_{\mathrm{gc}}$.

${ }^{13}$ For a more detailed discussion on the shift and angular momentum of paired states, see [30] and [179-181].
} 
to identify and distinguish the internal part from the guiding center part. Total angular momentum density generally does not satisfy this simple $1 / 2$ relation with Hall viscosity.

\subsection{Bulk and total Hall viscosities}

There is a further generalization of the above relation between Hall viscosity and internal angular momentum density. In $[49,171,172,174]$ a parity-odd thermodynamic transport coefficient $\tilde{\chi}_{\Omega}$ is defined as $T^{i j}=-\tilde{\chi}_{\Omega} \Omega g^{i j}+\ldots$ and it has been shown that

$$
\tilde{\chi}_{\Omega}=\frac{\delta P}{\delta \Omega} .
$$

$\tilde{\chi}_{\Omega}$ is called "Hall bulk viscosity" in [49] and "curl viscosity" in [172] (denoted by $\zeta_{A}$ there). Then we have

$$
\tilde{\chi}_{\Omega}=\frac{1}{2} \ell_{\mathrm{gc}} .
$$

As the Hall viscosity is (minus) half of the internal angular momentum density, this relation tells us that the Hall bulk viscosity is half the guiding center angular momentum density for fractional quantum Hall fluids. We can combine these two relations to find a relation with the total angular momentum density:

$$
\tilde{\chi}_{\Omega}-\eta_{H}=\frac{1}{2} \ell_{\text {tot }} .
$$

We can view this as a statement that the "total" parity-odd viscosity is half of the total angular momentum density in fractional quantum Hall fluids. The minus sign is just an accident of the definitions.

\subsection{Relations to Hall conductivity}

From (A.18) where $\Pi_{1}$ is just the Hall conductivity $\sigma_{H}$ defined in the usual way, $\ell_{\mathrm{gc}}\left(\tilde{\chi}_{\Omega}\right)$ enters as $O\left(\vec{\partial}^{2}\right)$ order coefficient:

$$
\sigma_{H}=\frac{\nu}{2 \pi}-\frac{1}{2 B} \frac{\delta \ell_{\mathrm{gc}}}{\delta B} \vec{\partial}^{2}+O\left(\epsilon^{3}\right)=\frac{\nu}{2 \pi}-\frac{1}{B} \frac{\delta \tilde{\chi}_{\Omega}}{\delta B} \vec{\partial}^{2}+O\left(\epsilon^{3}\right)
$$

The same numeric result is derived in [61], without explicit identification of $\ell_{\text {gc }}$. We can further compute the energy density $\left\langle\mathcal{E}^{0}\right\rangle$, internal pressure $P_{\text {int }}=\frac{1}{2} g_{i j}\left\langle T^{i j}\right\rangle$ and inverse internal compressibility $\kappa_{\text {int }}^{-1}=-\delta P_{\text {int }} / \delta \log V$, as defined in [48] (where $\left\langle\mathcal{E}^{0}\right\rangle$ is denoted by $\varepsilon$ ). They are computed directly from (2.36). For flat space with constant $B$ field and vanishing $E_{i}$ field, the results are

$$
\begin{aligned}
\left\langle\mathcal{E}^{0}\right\rangle & =-f_{0}[B]=-f[B]-\frac{\nu(\mathrm{g}-2)}{8 \pi m} B^{2}, \\
P_{\text {int }} & =f_{0}[B]-B f_{0}^{\prime}[B]=f[B]-B f^{\prime}[B]-\frac{\nu(\mathrm{g}-2)}{8 \pi m} B^{2}, \\
\kappa_{\text {int }}^{-1} & =-B^{2} f_{0}^{\prime \prime}[B]=-B^{2} f^{\prime \prime}[B]-\frac{\nu(\mathrm{g}-2)}{4 \pi m} B^{2} .
\end{aligned}
$$


The central column agrees with the relations given in [48]. For the scale-invariant cases such as that in (4.5), $B f^{\prime}[B]=2 f[B]$, the conformal Ward identity (2.38) is indeed satisfied. Using the right column, we get the following relation:

$$
\frac{1}{2} \frac{\delta \ell_{\mathrm{gc}}}{\delta \log B}=\eta_{H}-\frac{\mathrm{g}-2 s}{4}\left\langle J^{t}\right\rangle-\frac{m}{B} \kappa_{\mathrm{int}}^{-1} .
$$

Then

$$
\sigma_{H}=\frac{\nu}{2 \pi}-\frac{1}{B^{2}}\left(\eta_{H}-\frac{\mathrm{g}-2 s}{4}\left\langle J^{t}\right\rangle-\frac{m}{B} \kappa_{\text {int }}^{-1}\right) \vec{\partial}^{2}+O\left(\epsilon^{3}\right) .
$$

This is exactly the relation derived in [48] when $\mathrm{g}=s=0$, because there these parameters are not considered.

\subsection{Interpretation of two types of angular momentum density}

Now we present the overdue explanation of why there are two types of angular momentum density that we have identified from the total angular momentum density. For simplicity, in this subsection we will ignore the gyromagnetic factor $\mathrm{g}$ and intrinsic spin $s$, unless otherwise stated. Their existence do not change the physical interpretation the angular momentum densities, but add additional parts to their expressions, as can be see from the above results. Since the guiding center angular momentum density $\ell_{\mathrm{gc}}$ and vorticity $\Omega$ are closely related to the drift velocity $v^{i}$, which is a classical concept for particle moving in electromagnetic field, it is intuitive to start with classical mechanics, and then proceed to its quantum version.

\subsubsection{Classical charged particles in electromagnetic field}

Let us consider the classical motion of a charged particle moving in mutually parallel magnetic and electric fields. The Newtonian equation of motion is

$$
m \frac{d \vec{v}}{d t}=q(\vec{E}+\vec{v} \times \vec{B}) .
$$

By the following substitution:

$$
\vec{v}=\vec{v}_{\text {drift }}+\vec{v}_{\text {cycl }}, \quad \text { where } \quad \vec{v}_{\text {drift }}=\frac{\vec{E} \times \vec{B}}{B^{2}},
$$

the total kinetic motion described by velocity $\vec{v}$ is decomposed into two parts: a cyclotron motion $\vec{v}_{\text {cycl }}$ (with frequency $\omega=q B / m$ ) around a point center and a linear motion of the cyclotron center described by the drift velocity $\vec{v}_{\text {drift }}$. Similarly, the position vector of the particle can also be decomposed into these two parts

$$
\vec{x}=\vec{x}_{\mathrm{drift}}+\vec{x}_{\mathrm{cycl}}
$$

To be in agreement with the quantum Hall literature, we will call the cyclotron center the "guiding center". Thus $\vec{x}_{\text {drift }}$ and $\vec{v}_{\text {drift }}$ are the position and velocity of the guiding center and $\vec{x}_{\text {cycl }}$ and $\vec{v}_{\text {cycl }}$ describe the relative motion of the charged particle as seen in the guiding center frame. Furthermore, if we view the particle in cyclotron motion plus 
the magnetic flux enclosed by the circular orbit as a "composite particle" (in analog to the composite fermion theory [182] of fractional quantum Hall effect, but of course here is no quantization), then $\vec{x}_{\text {drift }}$ and $\vec{v}_{\text {drift }}$ describe the trajectorial motion of the center of mass of the composite particle. Meanwhile, $\vec{x}_{\mathrm{cycl}}$ and $\vec{v}_{\mathrm{cycl}}$ are no longer directly visible to an external observer, but they will manifest themselves as some internal properties of the composite particle. For example, the orbital angular momentum of the cyclotron motion in the guiding center frame, i.e. $m \vec{v}_{\text {cycl }} \times \vec{x}_{\text {cycl }}$, will now looks like the "intrinsic" spin of the composite particle (in addition to the actual intrinsic spin of the charged particle). This is the origin of our terminology "internal", which in classical sense means the "intrinsic to the composite particle", or "viewed in the guiding center frame". Thus the total angular momentum of the charged particle is the sum of the angular momentum of the guiding center and the internal angular momentum of the composite particle. Of course, here we have only been talking about a single particle. The generalization to fluid case is straightforward, and this explains the split between $\ell_{\mathrm{gc}}$ and $\ell_{\mathrm{int}}$ in $\ell_{\text {tot }}$ in classical sense. Particularly, if the velocity field of the composite particle fluid $\vec{v}_{\text {drift }}(\vec{x})$ has a non-vanishing curl $\vec{\Omega}=\nabla \times \vec{v}_{\text {drift }} \neq 0$ at some point, then this means the guiding centers are circulating around this point and the fluid will have some non-zero angular momentum density at this point. This is the guiding center angular momentum density $\ell_{\mathrm{gc}}$.

\subsubsection{Quantum mechanics: two Hilbert spaces}

The fractional quantum Hall effect is a complicated quantum many body problem with interactions between charged particles playing a huge role. The quantum picture is quite different from the classical one we have just discussed. However, there are structural similarities, and one of them is the split of the total Hilbert space into the guiding center part and cyclotron part, which is in direct analog to the classical case. A discussion on angular momentum and Hall viscosity from this perspective can be found first in [30] and later more systematically in $[18,50-52]$. Other related works and references can be found in the thesis review [183].

To discuss the structure of Hilbert space, let us first focus on 2-dimensional singleparticle quantum mechanics in the presence of perpendicular magnetic field. The coordinates $x^{i}$ and momentum $p_{i}=-i \partial_{i}$ form the 4 -dimensional phase space. The covariant momentum is $\pi_{i}=p_{i}-q A_{i}$ with $\left[\pi_{i}, \pi_{j}\right]=i q B \epsilon_{i j}$. Similar to the example in classical mechanics, $x^{i}$ can be split into the two parts

$$
x^{i}=R^{i}+\tilde{R}^{i}, \quad \text { where } \quad \tilde{R}^{i}=-\frac{\epsilon^{i j} \pi_{j}}{q B},
$$

where $R^{i}$ are the guiding center coordinates and $\tilde{R}^{i}$ the Landau orbit coordinates, the quantum version of our $\vec{x}_{\text {drift }}$ and $\vec{x}_{\text {cycl }}$. They satisfy commutation relations

$$
\begin{aligned}
& {\left[R^{i}, R^{j}\right]=-\frac{i}{q B} \epsilon^{i j},} \\
& {\left[\tilde{R}^{i}, \tilde{R}^{j}\right]=\frac{i}{q B} \epsilon^{i j},} \\
& {\left[R^{i}, \tilde{R}^{j}\right]=0 .}
\end{aligned}
$$


The 4-dimensional Hilbert space is now mapped into a tensor product of two copies of 2-dimensional Hilbert spaces whose phase space coordinates are $R^{i}$ and $\tilde{R}^{i}$ respectively. A notable feature of the two sub-Hilbert spaces is that within each one the two spatial coordinates are non-commutative $[20,21,184,185]$. As $\left\{R^{i}, R^{j}\right\}$ and $\left\{\tilde{R}^{i}, \tilde{R}^{j}\right\}$ form two sets of $\operatorname{SL}(2, \mathbb{R})$ algebra, the guiding center angular momentum $L_{\mathrm{gc}}$ and Landau orbit angular momentum $\tilde{L}$ can be defined as ${ }^{14}$

$$
L_{\mathrm{gc}}=\frac{1}{2} q B g_{i j}^{\mathrm{gc}} R^{i} R^{j}, \quad \tilde{L}=-\frac{1}{2} q B \tilde{g}_{i j} \tilde{R}^{i} \tilde{R}^{j},
$$

where according to [52] $g_{i j}^{\mathrm{gc}}$ and $\tilde{g}_{i j}$ are the guiding center and Landau orbit metrics. For us we always assume Galilean and rotational invariance, thus $g_{i j}^{\mathrm{gc}}=\tilde{g}_{i j}=g_{i j}$. It is straightforward to check that the total angular momentum defined in the usual way is the sum of the above two angular momenta:

$$
L_{\mathrm{tot}}=\epsilon^{i j} x_{i} p_{j}=L_{\mathrm{gc}}+\tilde{L} .
$$

$\tilde{L}$ is the angular momentum associated with the cyclotron motion and $L_{\mathrm{gc}}$ associated with the drift motion of the guiding center. In term of the more familiar language of creation and annihilation operators in symmetric gauge [20,21], we have

$$
\begin{aligned}
L_{\mathrm{tot}} & =b^{\dagger} b-a^{\dagger} a, \\
L_{\mathrm{gc}} & =b^{\dagger} b+\frac{1}{2}, \\
\tilde{L} & =-\left(a^{\dagger} a+\frac{1}{2}\right),
\end{aligned}
$$

Here our definitions of $a, a^{\dagger}, b$ and $b^{\dagger}$ follow those in [21]: $a$ and $a^{\dagger}$ shift the Landau level quantum number by one and $b$ and $b^{\dagger}$ shift the angular quantum number by one within each Landau level. It is very clear from these expressions that $\tilde{L}$ is associated with the cyclotron motion and $L_{\mathrm{gc}}$ is the rest part of the motion. In fact, $\tilde{L}$ is proportional to the free Hamiltonian $H_{0}=\frac{q B}{m}\left(a^{\dagger} a+\frac{1}{2}\right)$.

So far we have only talked about single-particle quantum mechanics, for simplicity of the definitions. In the discussion of $[18,50-52]$ they are all generalized to many-particle case with interactions. The eigenvalue of (many-particle) Landau orbit angular momentum $\tilde{L}$ is extensive, while that of guiding center $L_{\mathrm{gc}}$ (thus $L_{\text {tot }}$ as well) has both super-extensive and extensive parts. ${ }^{15}$ The notion of angular momentum density can be defined from the extensive part of the angular momentum. Then the coefficients of extensive part of $L_{\mathrm{gc}}$, $\tilde{L}$ and $L_{\text {tot }}$ define the guiding center, Landau orbit and total angular momentum density $\ell_{\mathrm{gc}}, \tilde{\ell}$ and $\ell_{\text {tot }}$, respectively. Divided by $\left\langle J^{t}\right\rangle, \ell_{\mathrm{gc}}$ and $\tilde{\ell}$ are minus the "guiding center spin" (denoted by $s$ in [52], not to be confused with our $s$ ) and "Landau orbit spin" $\tilde{s}_{n}$ defined in [52]. $\ell_{\mathrm{gc}}$ is exactly the quantity we defined and computed earlier, and $\tilde{\ell}$ only differs

\footnotetext{
${ }^{14}$ Our definition of $L_{\mathrm{gc}}$ differs by a sign from that in [52] such that its positive direction aligns in the same direction as $\tilde{L}$ 's. In [52] they align in opposite directions because their two sets of $\operatorname{SL}(2, \mathbb{R})$ algebra, eq. (6a) and eq. (6b), differ by a sign.

15 "Extensive" means proportional to the total electron number $N$, and "super-extensive" quadratic in $N$.
} 
from our $\ell_{\text {int }}$ by the intrinsic spin $s$, which we have ignored here. This is the quantum interpretation of the meaning of what we called guiding center angular momentum density $\ell_{\mathrm{gc}}$ and internal angular momentum density $\ell_{\text {int }}$.

In additional to deriving the analog of the relation (5.16) for $\tilde{\ell}$, [52] defines a guiding center Hall viscosity and shows that it is proportional to the guiding center spin. This is the analog of our (5.18). By comparison, the guiding center Hall viscosity is essentially the bulk Hall viscosity $\tilde{\chi}_{\Omega}$. [52] also has an equation that the sum of the two Hall viscosities equals to the sum ${ }^{16}$ of the guiding center and Landau orbit spins. This is the analog of our relation (5.19). Our results can be viewed as a derivation of these results from Chern-Simons effective theory.

\subsection{Guiding center momentum density}

Since $v^{i}$ has been identified with drift velocity, i.e. the guiding center velocity field, its natural to identify the "charge density" conjugate to it as the guiding center momentum density $p_{\mathrm{gc}}^{i}$, similar to the relation between $\ell_{\mathrm{gc}}$ and $\Omega$ :

$$
\left\langle p_{\mathrm{gc}}^{i}\right\rangle=\left.\frac{e^{\Phi}}{\sqrt{g}} \frac{\delta \mathcal{I}}{\delta v_{i}}\right|_{A_{\mu}, g_{i j}, \Phi} .
$$

Notice that this equation shall be computed before the path-integral constraint or field constraint is implemented, otherwise there would be no $v_{i}$ at all and the quasi-effective action $\mathcal{I}\left[v^{i}, A_{\mu}, g_{i j}, \Phi, \ldots\right]$ would become the effective action $\mathcal{W}\left[A_{\mu}, g_{i j}, \Phi, \ldots\right]$. In other words, $\left\langle p_{\text {gc }}^{i}\right\rangle$ is computed from the quasi-effective potential $\mathcal{I}$, not the actual effective potential $\mathcal{W}$. In this sense, we can not say that $v_{i}$ is a source field that sources certain momentum density, because source fields are the independent functional variables appearing in $\mathcal{W}$. This is a consequence that there is no $v_{i}$ in the microscopic action (2.7). The above equation can be viewed as a definition for $\left\langle p_{\mathrm{gc}}^{i}\right\rangle$, and there is no conservation law associated with it. This is different from the total momentum density (2.37), which enters the conservation of stress-tensor [60]. On contrary, quantity like $v_{i}$ does enter effective action (and even the microscopic action) as a source field to momentum density $p^{i}$ in some formalisms in the literature, for example in $[60,64,72]$. This is case, the definition of $A_{i}$ is also shifted (usually by $m v_{i}$ ), then the current $J^{i}$ obtained through functional derivative with respect to $A_{i}$ has not exactly the same meaning as ours defined in (2.36). It is related to our current by some redefinition, as illustrated in [60]. Thus a relation like $p^{i}=m J^{i}$ usually given in this case (where $p^{i}$ is obtained from functional derivative with respect to $v_{i}$ ) is in no contradiction with any relation presented in this paper.

Using (4.6) and (4.7), we have

$$
\begin{aligned}
\left\langle p_{\mathrm{gc}}^{i}\right\rangle= & \frac{\nu}{2 \pi}\left\{m e^{2 \Phi}\left(\varepsilon^{i j} E_{j}-B v^{i}\right)-\varepsilon^{i j} \partial_{j}\left[\left(1-\frac{\mathrm{g}}{4}+\frac{s^{\prime}}{2}\right) B\right]\right. \\
& \left.+\left(1-\frac{\mathrm{g}}{4}+\frac{s^{\prime}}{2}\right) e^{\Phi} B \varepsilon^{i j} \partial_{j} \log \left(m e^{\Phi}\right)\right\}+m e^{2 \Phi} \varepsilon^{i j} \partial_{j}\left(e^{-\Phi} f^{\prime}[B]\right) \\
& +O\left(\epsilon^{2}\right) .
\end{aligned}
$$

\footnotetext{
16 "Minus" in [52] again because their definitions of the two angular momenta differ by a sign.
} 
For $\Phi=0$ and $m, \mathrm{~g}, s$ and $s^{\prime}$ constant, it becomes

$$
\left\langle p_{\mathrm{gc}}^{i}\right\rangle=\varepsilon^{i j} \partial_{j}\left\{-\frac{\nu}{2 \pi}\left(1-\frac{\mathrm{g}}{4}+\frac{s^{\prime}}{2}\right) B+m f^{\prime}[B]\right\}+O\left(\epsilon^{2}\right) .
$$

It has no "transport" part ${ }^{17}$ and the only non-vanishing part is a curl which accounts exactly for the guiding center angular momentum density (5.13). Subtracting it from the total momentum density (5.6), we obtain the rest, the internal momentum density:

$$
\left\langle p_{\text {int }}^{i}\right\rangle=\frac{\nu m}{2 \pi} \varepsilon^{i j} E_{j}+\varepsilon^{i j} \partial_{j}\left[\frac{\nu}{4 \pi}\left(s+s^{\prime}\right) B\right]+O\left(\epsilon^{2}\right) .
$$

The second term is the curl part which accounts for all the internal angular momentum density (5.15), as expected. The first term is the transport part, which is a product of number density $\left\langle J^{t}\right\rangle$, mass $m$ and the drift velocity $\varepsilon^{i j} E_{j} / B$. This form is exactly what one would expect from classical mechanics. Notably, this accounts for all the transport part of the total momentum density in the homogeneous case.

\section{Summary and comments}

In this paper we have shown that Hořava-Lifshitz gravity theory can be used as a covariant formalism for the effective field theory of the fractional quantum Hall effect in $2+1$ dimensions. It guarantees the non-relativistic spacetime symmetries possessed by the quantum Hall system. Lying in the heart of this formalism is the map between the field degrees of freedom of the two theories, (3.38)-(3.45). We originally derive this map as a holographic dictionary, but its existence and form are independent of the holographic duality. It can be directly applied on (2+1)-dimensional Hořava-Lifshitz gravity theory to construct the low energy effective action for the fractional quantum Hall effect in a phenomenological way. It also serves as the holographic dictionary for $(3+1)$-dimensional Hořava-Lifshitz gravity theory with asymptotic Lifshitz background (3.18). The latter paves the way for a systematic study on the fractional quantum Hall effect using the approach of gauge/gravity duality (AdS/CMT), that has been well-developed over the last decade. Of particular interest is that this formalism naturally produces the Wen-Zee term at the boundary, which is otherwise hard to obtain using the conventional relativistic (3+1)-dimensional holography.

We have shown that the universal electromagnetic and geometric properties of the fractional quantum Hall effect that are previously studied over decades in various contexts using different methods can be encoded in a simple Chern-Simons model using the HořavaLifshitz gravity formalism. The effective action we obtain in this paper is by no means a complete one. Additional terms can be added phenomenologically based on symmetry considerations, but the connection of Hořava-Lifshitz formalism to holography allows them to be computed systematically using gauge/gravity duality. The shift function in HořavaLifshitz gravity is identified with (minus) the guiding center velocity field (drift velocity) of the quantum Hall fluid, whose conjugate momentum density is also that of the guiding center. Through this identification, we can further identify the guiding center angular

\footnotetext{
${ }^{17}$ According to the terminology of [176], this is the part that can not be written as a curl.
} 
momentum density conjugate to the guiding center vorticity is half of the Hall bulk viscosity defined in the context of parity-violating first order hydrodynamics [49, 171, 174]. The rest of the total angular momentum density is the internal angular momentum density of the composite particles, which includes the Landau orbit spin and the intrinsic spin of the composite particles. It is proportional to the Wen-Zee shift and is minus twice of the Hall viscosity. These relations are well-known in the literature and are derived using various methods $[30,47,48]$. Here we show that they can be produced via non-relativistic diffeomorphism invariant Chern-Simons effective field theory.

We close our paper with some concluding remarks and comments:

1. Various versions of Hor̆ava-Lifshitz gravity: there exist different versions of HořavaLifshitz gravity theory in the literature. What we employ here is a minimal version of the non-projectable Hořava-Lifshitz gravity, where the graviton sector includes only the lapse function $N$, shift function $N_{i}$ (or $N_{i}$ ) and spatial metric $g_{i j}\left(\right.$ or $G_{I J}$ ), all being functions of both spatial coordinates and time, and the spacetime symmetries contain primarily only the foliation preserving diffeomorphism (FPD) and Weyl scaling. There are extended versions of the theory, notably that in $[132,133]$. There an additional $\mathrm{U}(1)_{\Sigma}$ gauge symmetry associated with the co-dimension one foliation of constant time $\Sigma(t)$ is introduced. This is not to be confused with the electromagnetic $\mathrm{U}(1)$ symmetry represented by the vector field $V_{\mu}$ or $V_{M}$ in this paper. But sometimes they are identified in the literature, for example in $[59,66]$. Consequently additional fields are needed to construct a manifest $\mathrm{U}(1)_{\Sigma}$ gauge-invariant theory. This kind of extended version of Hořava-Lifshitz gravity can also be used as a bulk theory for holography [117] as well as covariant formalism for quantum Hall effects. The covariant map can be built in a similar way as we have done in this paper to incorporate the additional fields. But what are the field theory dual of this additional symmetry and fields is a question that usually has no obvious answer, at least in the context of quantum Hall effects. [117] gives two possible holographic scenarios for the extended version with $\mathrm{U}(1)_{\Sigma}$ symmetry. We choose to use the minimal version without $\mathrm{U}(1)_{\Sigma}$ symmetry symmetry and additional fields. A fundamental reason behind these different choices is how the shift function is interpreted in the field theory, because the $\mathrm{U}(1)_{\Sigma}$ is an Abelian gauge symmetry of the shift function. If the shift function is treated like a gauge field in the dual field theory, such as in $[59,66]$, it is natural to include this symmetry and employ the $\mathrm{U}(1)_{\Sigma}$ extended version of HořavaLifshitz gravity as bulk theory, following [117]'s prescription regarding the role of this symmetry and additional fields in the holographic correspondence. However, if the shift function is interpreted as a velocity field, as in [61] and this paper, it is more of the nature of a current ${ }^{18}$ which does not transform under a $U(1)$ symmetry. In this case, the $\mathrm{U}(1)_{\Sigma}$ extended version will not help much in building the effective theory based on symmetries. Thus we choose the minimal version.

\footnotetext{
${ }^{18}$ We recall in non-relativistic theories, current $\vec{j}$ is proportional to velocity $\vec{v}$, with coefficient being the density $\rho: \vec{j}=\rho \vec{v}$.
} 
2. Behavior of the shift function: different versions of Hořava-Lifshitz gravity may have different dynamics and numbers of degrees of freedom, depending on the numbers of fields and numbers of first-class and second-class constraints (for example, see [186189]). Even in the low-energy minimal version we use here, there are still free tunable parameters $\alpha, \beta$ and $\hat{\lambda}$ in the action (1.5), which can give rise to different dynamics and solutions. Thus the shift function (or the boundary field of the shift function in the case of holography) can have different behaviors depending on what the model action one start with. This feature is particularly relevant for holography. This does not affect the the holographic dictionary since the symmetries are the same, but gives different physical results. In some versions of Hořava-Lifshitz gravity, the shift function (to be precise, its boundary field in holography) may totally drop off after the holographic dictionary is applied. This is suggested as one scenario in [117]. In some other versions, it may be constrained to be just a functional of other fields. In either case, the question of how to deal with it is trivial. We do not focus on these cases in this paper, but are aware of these possibilities. What we are mostly interested in is the case that the boundary shift function is independent of the other boundary fields. This is similar to that of the relativistic holography. From the computation we have done, it also seems to be the most relevant case for application to quantum Hall effects. It gives rise to the non-trivial questions of how to interpret and determine the shift function. This leads to the next comment. We hope to give some case-by-case study in the future to show some examples of what model actions can give what possible cases among these three possibilities for the shift function.

3. The two constraints: we identify the shift function arising in Hořava-Lifshitz formalism as minus the guiding center velocity field, but how to determine it is a different question. Similar problem exists in the Newton-Cartan formalism as well. There is no clear answer to this question in recent studies of the quantum Hall effective action, among which many do not provide an answer at all. In this paper, we provide two slightly different answers, the path-integral constraint (3.51) and the field constraint (3.52), both satisfying the requirements from symmetries and LLL projection. They are both meaningful and applicable in $(2+1)$-dimensional effective field theory and (3+1)-dimensional holography. The former is more in the spirit of effective theory, similar to what is done in [61], while the latter is more commonly seen in holography as a Dirichlet boundary condition imposed at the boundary. For the fractional quantum Hall effect where at low energy scale the Chern-Simons term dominates, the two constraints are equivalent in producing the universal features, but can give different results for non-universal features related to local dynamics. The question that which one is more favorable has to be answered by computing more non-universal features and comparing with other approaches and the experimental results. It is beyond the reach of this paper. It is worthy to note that in the absence of gauge Chern-Simons term and strong background magnetic field, i.e. out of the context of quantum Hall effects (e.g. in the context of [117]), these two constraints will yield quite different results. 
4. (2+1)-dimensional Chern-Simons effective theory: the fractional quantum Hall effective action we obtain in this paper is through holographic non-dynamical ChernSimons model in $3+1$ dimensions. This is the simplest holographic model one can write down for the fractional quantum Hall effect. But the same results can be equally obtained without holography, by just starting with a $(2+1)$-dimensional Chern-Simons model, as is typically done in the traditional effective field theory approach. The reason is that the non-dynamical Chern-Simons terms are boundary terms in holography. From this point of view, it seems holography does not make a difference. This is only true so far as only the non-dynamical Chern-Simons terms and the universal features of certain fixed fractional quantum Hall states are concerned, and the universality of quantum Hall physics at low energy guarantees that all methods converge at this point. The true power of holography really lies in the analysis of local dynamics which governs the non-universal properties, and of quantum phase transitions such as the transitions between different Hall plateaus. This leads to the next two remarks.

5. Local dynamics: the majority of the quantum Hall research focuses on the universal properties that is related to topology and Chern-Simons field theory, so is the ChernSimons model studied in this paper. Properties related to local dynamics such as the Coulomb interaction are much harder to study for such a strongly correlated quantum system and the methods available are quite limited. Holography offers a strong and efficient toolkit to deal with such a difficulty, and the holographic dictionary we build in this paper opens the doorway to it. It will be interesting to solve a specific holographic Hořava-Lifshitz model with local dynamics, for example, specified by (1.5) and (1.6), maybe with additional scalar fields, to see what local dynamics and non-universal properties it can generate for the fractional quantum Hall effect. We will leave this in a forthcoming study.

6. Holographic dynamical Chern-Simons and axion-dilaton models: in the nondynamical Chern-Simons model we have studied in this paper, the coefficient of the gauge Chern-Simons term is a constant. It can be turned into a dynamical axion field and thus enters the bulk dynamics. So is the coefficient of the Maxwell action (1.6) that can be turned into a dynamical dilaton. The relativistic version of this model and its variations have been studied in $[104,107]$. The additional $\operatorname{SL}(2, \mathbb{Z})$ symmetry of the axion-dilation system captures the transport features of the Hall plateau transitions. This is another example of Holography as a powerful tool for studying quantum phase transitions. A similar study can be carried out using Hořava-Lifshitz holography using our holographic dictionary. We expect it will give more information and features, both universal and non-universal, than the simple Chern-Simons model we have studied here.

7. Global time and energy flux: in this paper, we work exclusively in the global time coordinates and prohibit the foliation mixing diffeomorphism $\partial_{i} \xi^{t} \neq 0$. This keeps our formalism simple and convenient. But a major drawback is the lack of the source field for energy flux, thus our current formalism can not compute energy flux. The 
source can be restored in non-relativistic field theory [60] and dealt with in nonrelativistic holography by introducing additional vector field in the bulk as shown in [117]. A closely related question is about the existence of global time. Clearly it is needed for any genuine non-relativistic theory as part of the causal structure. But for holography, this does not necessarily imply the existence of global time in the whole bulk, as long as there is another mechanism that provides the global time structure on the boundary. In this paper, we assume global time for the whole bulk and thus employ the Hořava-Lifshitz gravity theory as the holographic dual. This is a simple and convenient choice conceptually, and practically is enough for many purposes as long as the energy flux is not concerned. But there can be other choices. For example, [94, 95] has shown that certain $z=2$ Lifshitz holography with relativistic bulk theory can have a boundary whose geometry can be identified with various versions of non-relativistic Newton-Cartan geometry, depending on the boundary condition for the time-like vielbein. In a forthcoming paper we will discuss the possibility of giving up bulk global time and restoring the energy flux source and try to generalize our formalism to a relativistic bulk.

8. Generalization to multiple species / hierarchical states: our formalism, starting from the microscopic field theory action (2.7), describes a single species of spin-polarized particles carrying mass $m$, Abelian charge $e$ (which has been set to unity in this paper), gyromagnetic factor $g$ and intrinsic spin $s$. It thus mostly naturally describe the Laughlin states of fractional quantum Hall fluids with inverse filling factor equal to an odd integer. A large number of other quantum Hall states can be described by Abelian hierarchical states [11, 170, 182] where different types of quantum Hall fluids of quasiholes/quasiparticles coexist. A field theoretical framework, the K-matrix formulation, is introduced in [5]. In this formulation, the Abelian gauge fields carry additional flavor index; the charges becomes a flavor space vector and Chern-Simons couplings a matrix - the K-matrix. Such a formulation is employed recently in the construction of low energy effective action in $[67,68]$. To accommodate this picture, our formalism need to be extended to include multiple species of particles carrying different characteristics such as mass and charge. Since the non-relativistic spacetime symmetries (2.10)-(2.15) and (2.19)-(2.25) depend on parameters such as mass and charge, they may need modifications first. Thus this generalization is quite nontrivial. Generalization to multi-species in non-relativistic holography is a non-trivial question as well. A first trial is given in [113], but this construction involves adding more extra dimensions to the bulk, which is not a method suitable to the approach we employ in this paper. We will leave this task to a forthcoming study.

\section{Acknowledgments}

We are very grateful to Dam Thanh Son for constant support and inspiring discussions throughout the progress of this work. We thank Andreas Karch for reading the draft of this paper and making valuable and encouraging comments. We also thank Stefan Janiszewski and Kristan Jensen for useful discussions during their visits to EFI. This work 
is supported, in part, by the US DOE grant No. DE-FG02-13ER41958, NSF DMS-1206648 and a Simons Investigator grant from the Simons Foundation. S.-F. Wu is supported by NNSFC No. 11275120 and the China Scholarship Council.

\section{A General formulae for correlation functions}

If the effective action takes the following form:

$$
\begin{aligned}
\mathcal{W}= & \int d t d^{2} \vec{x}\left\{\frac{\nu}{4 \pi} \epsilon^{\rho \mu \nu} A_{\rho} \partial_{\mu} A_{\nu}-\frac{\nu}{2 \pi}\left(s+s^{\prime}\right) \epsilon^{\rho \mu \nu} A_{\rho} \partial_{\mu} \omega_{\nu}+\frac{\nu}{4 \pi}\left(s+s^{\prime}\right)^{2} \epsilon^{\rho \mu \nu} \omega_{\rho} \partial_{\mu} \omega_{\nu}\right\} \\
& +\int d t d^{2} \vec{x} \sqrt{g}\left\{\alpha\left[B, E^{2}\right]+f_{0}\left[B, \mathcal{R}^{(2)}\right]+f_{1}[B] E^{i} \nabla_{i} \log B\right. \\
& \left.+f_{2}[B]\left(\nabla_{i} \log B\right)^{2}+O\left(\epsilon^{3}\right)\right\} .
\end{aligned}
$$

with $\Phi=0$ and $m, \mathrm{~g}, s$ and $s^{\prime}$ are all constants, then the correlation functions defined by (2.36) can be computed as following. The non-equilibrium 1-point function of the conserved current is

$$
\begin{aligned}
\left\langle J^{t}\right\rangle= & \frac{\nu}{2 \pi}\left[B-\left(s+s^{\prime}\right) \mathcal{R}^{(2)}\right]-\nabla_{i}\left(2 E^{i} \frac{\delta \alpha}{\delta E^{2}}+f_{1} \nabla^{i} \log B\right)+O\left(\epsilon^{3}\right) \\
\left\langle J^{i}\right\rangle= & \frac{\nu}{2 \pi} \varepsilon^{i j}\left[E_{j}-\left(s+s^{\prime}\right)\left(\partial_{j} \omega_{t}-\partial_{t} \omega_{j}\right)\right]+\frac{1}{\sqrt{g}} \partial_{t}\left[\sqrt{g}\left(2 E^{i} \frac{\delta \alpha}{\delta E^{2}}+f_{1} \nabla^{i} \log B\right)\right] \\
& +\varepsilon^{i j} \nabla_{j}\left[\alpha^{\prime}-\frac{f_{1}}{B} \nabla_{k} E^{k}+f_{0}^{\prime}-f_{2}^{\prime}\left(\nabla_{i} \log B\right)^{2}-2 \frac{f_{2}}{B} \nabla^{2} \log B\right]+O\left(\epsilon^{4}\right), \\
= & \frac{\nu}{2 \pi} \varepsilon^{i j}\left[E_{j}-\left(s+s^{\prime}\right)\left(\partial_{j} \omega_{t}-\partial_{t} \omega_{j}\right)\right]+\frac{1}{\sqrt{g}} \partial_{t}\left[\sqrt{g}\left(2 E^{i} \frac{\delta \alpha}{\delta E^{2}}+f_{1} \nabla^{i} \log B\right)\right] \\
& +\left\{\alpha^{\prime \prime}+f_{0}^{\prime \prime}-\left(\frac{f_{1}}{B}\right)^{\prime} \nabla_{k} E^{k}-2\left(\frac{f_{2}}{B^{2}}\right)^{\prime} \nabla^{2} B-\left[f_{2}^{\prime \prime}-2\left(\frac{f_{2}}{B}\right)^{\prime}\right]\left(\nabla_{k} \log B\right)^{2}\right\} \varepsilon^{i j} \nabla_{j} B \\
& -2 \frac{f_{2}}{B} \varepsilon^{i j} \nabla_{j} \nabla^{2} B-\left(f_{2}^{\prime}-2 \frac{f_{2}}{B}\right) \varepsilon^{i j} \nabla_{j}\left[\left(\nabla_{k} \log B\right)^{2}\right] \\
& +\frac{\delta \alpha^{\prime}}{\delta E^{2}} \varepsilon^{i j} \nabla_{j} E^{2}-\frac{f_{1}}{B} \varepsilon^{i j} \nabla_{j} \nabla_{k} E^{k}+O\left(\epsilon^{4}\right),
\end{aligned}
$$

where "'” is derivative with respect to $B$. The 2-point functions are

$$
\begin{aligned}
\left\langle J^{t}(x) J^{t}(0)\right\rangle= & i \nabla^{i}\left[2 \frac{\delta \alpha}{\delta E^{2}} \partial_{i} \delta(x)\right]+O\left(\epsilon^{3}\right) \\
\left\langle J^{t}(x) J^{i}(0)\right\rangle= & i \frac{\nu}{2 \pi} \varepsilon^{i j} \partial_{j} \delta(x)-2 i \nabla^{i}\left[\frac{\delta \alpha}{\delta E^{2}} \partial_{t} \delta(x)\right] \\
& -i \varepsilon^{i j} \nabla^{k}\left[\left(2 E_{k} \frac{\delta \alpha^{\prime}}{\delta E^{2}}+f_{1}^{\prime} \nabla_{k} \log B\right) \partial_{j} \delta(x)+f_{1} \nabla_{k}\left(\frac{\partial_{j} \delta(x)}{B}\right)\right]+O\left(\epsilon^{4}\right), \\
\left\langle J^{i}(x) J^{t}(0)\right\rangle= & -i \frac{\nu}{2 \pi} \varepsilon^{i j} \partial_{j} \delta(x)-\frac{2 i}{\sqrt{g}} \partial_{t}\left[\sqrt{g} \frac{\delta \alpha}{\delta E^{2}} \partial^{i} \delta(x)\right] \\
& -i \varepsilon^{i j} \nabla_{j}\left[2 E^{k} \frac{\delta \alpha^{\prime}}{\delta E^{2}} \partial_{k} \delta(x)-\frac{f_{1}}{B} \nabla^{2} \delta(x)\right]+O\left(\epsilon^{4}\right)
\end{aligned}
$$




$$
\begin{aligned}
\left\langle J^{i}(x) J^{j}(0)\right\rangle= & i \frac{\nu}{2 \pi} \varepsilon^{i j} \partial_{t} \delta(x)+\frac{i}{\sqrt{g}} \partial_{t}\left[2 \sqrt{g} \frac{\delta \alpha}{\delta E^{2}} g^{i j} \partial_{t} \delta(x)\right] \\
& +\frac{i}{\sqrt{g}} \partial_{t}\left\{\sqrt{g} \varepsilon^{j k}\left[\left(2 E^{i} \frac{\delta \alpha^{\prime}}{\delta E^{2}}+f_{1}^{\prime} \nabla^{i} \log B\right) \partial_{k} \delta(x)+f_{1} \nabla^{i}\left(\frac{\partial_{k} \delta(x)}{B}\right)\right]\right\} \\
& +i \varepsilon^{i k} \nabla_{k}\left[2 E^{j} \frac{\delta \alpha^{\prime}}{\delta E^{2}} \partial_{t} \delta(x)-\frac{f_{1}}{B} \partial^{j} \partial_{t} \delta(x)\right] \\
& +i \varepsilon^{i k} \varepsilon^{j l} \nabla_{k}\left\{\left[\alpha^{\prime \prime}-\left(\frac{f_{1}}{B}\right)^{\prime} \nabla \cdot \vec{E}+f_{0}^{\prime \prime}-f_{2}^{\prime \prime}(\nabla \log B)^{2}\right.\right. \\
& \left.\left.-2\left(\frac{f_{2}}{B}\right)^{\prime} \nabla^{2} \log B\right] \partial_{l} \delta(x)\right\} \\
& -i \varepsilon^{i k} \varepsilon^{j l} \nabla_{k}\left[2 f_{2}^{\prime}\left(\nabla^{n} \log B\right) \nabla_{n}\left(\frac{\partial_{l} \delta(x)}{B}\right)+2 \frac{f_{2}}{B} \nabla^{2}\left(\frac{\partial_{l} \delta(x)}{B}\right)\right]+O\left(\epsilon^{5}\right),
\end{aligned}
$$

where we have assumed $\delta^{2} \alpha /\left(\delta E^{2}\right)^{2}=0$. For flat background with constant $B$ and $E_{i}$ fields, the 2-point functions simplify to

$$
\begin{aligned}
\left\langle J^{t}(x) J^{t}(0)\right\rangle= & 2 i \frac{\delta \alpha}{\delta E^{2}} \vec{\partial}^{2} \delta(x)+O\left(\epsilon^{3}\right), \\
\left\langle J^{t}(x) J^{i}(0)\right\rangle= & i \frac{\nu}{2 \pi} \epsilon^{i j} \partial_{j} \delta(x)-2 i \frac{\delta \alpha}{\delta E^{2}} \partial^{i} \partial_{t} \delta(x) \\
& -i \epsilon^{i j}\left[2 \frac{\delta \alpha^{\prime}}{\delta E^{2}} E^{k} \partial_{k} \partial_{j} \delta(x)+\frac{f_{1}}{B} \vec{\partial}^{2} \partial_{j} \delta(x)\right]+O\left(\epsilon^{4}\right), \\
\left\langle J^{i}(x) J^{t}(0)\right\rangle= & -i \frac{\nu}{2 \pi} \epsilon^{i j} \partial_{j} \delta(x)-2 i \frac{\delta \alpha}{\delta E^{2}} \partial^{i} \partial_{t} \delta(x) \\
& -i \epsilon^{i j}\left[2 \frac{\delta \alpha^{\prime}}{\delta E^{2}} E^{k} \partial_{k} \partial_{j} \delta(x)-\frac{f_{1}}{B} \vec{\partial}^{2} \partial_{j} \delta(x)\right]+O\left(\epsilon^{4}\right), \\
\left\langle J^{i}(x) J^{j}(0)\right\rangle= & i \frac{\nu}{2 \pi} \epsilon^{i j} \partial_{t} \delta(x)+2 i \delta^{i j} \frac{\delta \alpha}{\delta E^{2}} \partial_{t}^{2} \delta(x)-i \epsilon^{i j} \frac{f_{1}}{B} \vec{\partial}^{2} \partial_{t} \delta(x) \\
& +2 i\left(\epsilon^{j k} E^{i}+\epsilon^{i k} E^{j}\right) \frac{\delta \alpha^{\prime}}{\delta E^{2}} \partial_{k} \partial_{t} \delta(x) \\
& +i\left(\alpha^{\prime \prime}+f_{0}^{\prime \prime}-2 \frac{f_{2}}{B^{2}} \vec{\partial}^{2}\right)\left(\delta^{i j} \vec{\partial}^{2}-\partial^{i} \partial^{j}\right) \delta(x)+O\left(\epsilon^{5}\right) .
\end{aligned}
$$

They have the following structures:

$$
\begin{aligned}
\left\langle J^{t}(x) J^{t}(0)\right\rangle= & i \Pi_{0} \vec{\partial}^{2} \delta(x), \\
\left\langle J^{t}(x) J^{i}(0)\right\rangle= & -i \Pi_{0} \partial^{i} \partial_{t} \delta(x)+i \Pi_{1} \epsilon^{i j} \partial_{j} \delta(x)-i \Pi_{3} \epsilon^{i j} E^{k} \partial_{k} \partial_{j} \delta(x), \\
\left\langle J^{i}(x) J^{t}(0)\right\rangle= & -i \Pi_{0} \partial^{i} \partial_{t} \delta(x)-i \Pi_{1} \epsilon^{i j} \partial_{j} \delta(x)-i \Pi_{3} \epsilon^{i j} E^{k} \partial_{k} \partial_{j} \delta(x), \\
\left\langle J^{i}(x) J^{j}(0)\right\rangle= & i \Pi_{0} \delta^{i j} \partial_{t}^{2} \delta(x)+i \Pi_{1} \epsilon^{i j} \partial_{t} \delta(x)+i \Pi_{2}\left(\delta^{i j} \vec{\partial}^{2}-\partial^{i} \partial^{j}\right) \delta(x) \\
& +i \Pi_{3}\left(\epsilon^{j k} E^{i}+\epsilon^{i k} E^{j}\right) \partial_{k} \partial_{t} \delta(x),
\end{aligned}
$$


with

$$
\begin{aligned}
& \Pi_{0}=2 \frac{\delta \alpha}{\delta E^{2}}+O(\epsilon) \\
& \Pi_{1}=\frac{\nu}{2 \pi}-\frac{f_{1}}{B} \vec{\partial}^{2}+O\left(\epsilon^{3}\right), \\
& \Pi_{2}=\alpha^{\prime \prime}+f_{0}^{\prime \prime}-2 \frac{f_{2}}{B^{2}} \vec{\partial}^{2}+O\left(\epsilon^{3}\right), \\
& \Pi_{3}=2 \frac{\delta \alpha^{\prime}}{\delta E^{2}}+O(\epsilon) .
\end{aligned}
$$

Notice that $\Pi_{1}$ is actually the Hall conductivity $\sigma_{H}$ defined in the usual way. The above expressions of 2-point functions are usually written in momentum space, where $\partial_{t} \rightarrow-i \omega$, $\partial_{i} \rightarrow i k_{i}$ and $\delta(x) \rightarrow 1$.

\section{B Near-boundary vectorial structures and diffeomorphism}

There are additional vectorial structures we can make up using the boundary fields $\bar{N}, \bar{N}_{i}$, $\bar{G}_{i j}$ and $\bar{\phi}$. Their diffeomorphism transformations at the boundary are

$$
\begin{aligned}
\delta\left(\bar{\varepsilon}^{j k} \partial_{j} \bar{N}_{k}\right)= & \bar{\xi}^{\mu} \partial_{\mu}\left(\bar{\varepsilon}^{j k} \partial_{j} \bar{N}_{k}\right)+\left(\bar{\varepsilon}^{j k} \partial_{j} \bar{N}_{k}\right) \partial_{t} \bar{\xi}^{t} \\
& +\bar{\varepsilon}^{j k} \partial_{j}\left(\bar{G}_{k l} \partial_{t} \bar{\xi}^{l}\right)+2 \bar{\varepsilon}^{j k} \bar{N}_{k} \partial_{j} \bar{\sigma}, \\
\delta\left(\bar{\varepsilon}^{j k} \bar{N}_{k} \partial_{j} \log \frac{\bar{N}}{\bar{\phi}}\right)= & \bar{\xi}^{\mu} \partial_{\mu}\left(\bar{\varepsilon}^{j k} \bar{N}_{k} \partial_{j} \log \frac{\bar{N}}{\bar{\phi}}\right)+\left(\bar{\varepsilon}^{j k} \bar{N}_{k} \partial_{j} \log \frac{\bar{N}}{\bar{\phi}}\right) \partial_{t} \bar{\xi}^{t} \\
& +\left(\bar{\varepsilon}^{j k} \bar{G}_{k l} \partial_{j} \log \frac{\bar{N}}{\bar{\phi}}\right) \partial_{t} \bar{\xi}^{l}+\left(z-\Delta_{\phi}\right) \bar{\varepsilon}^{j k} \bar{N}_{k} \partial_{j} \bar{\sigma}, \\
\delta\left(\bar{\varepsilon}^{j k} \bar{G}_{k i} \partial_{j} \log \frac{\bar{N}}{\bar{\phi}}\right)= & \bar{\xi}^{\mu} \partial_{\mu}\left(\bar{\varepsilon}^{j k} \bar{G}_{k i} \partial_{j} \log \frac{\bar{N}}{\bar{\phi}}\right)+\left(\bar{\varepsilon}^{j k} \bar{G}_{k l} \partial_{j} \log \frac{\bar{N}}{\bar{\phi}}\right) \partial_{i} \bar{\xi}^{l} \\
& +\left(z-\Delta_{\phi}\right) \bar{\varepsilon}^{j k} \bar{G}_{k i} \partial_{j} \bar{\sigma} \cdot \\
\delta\left(\bar{\phi} \frac{\bar{G}^{j k} \bar{N}_{j} \bar{N}_{k}}{2 \bar{N}}=\right. & \bar{\xi}^{\mu} \partial_{\mu}\left(\bar{\phi} \frac{\bar{G}^{j k} \bar{N}_{j} \bar{N}_{k}}{2 \bar{N}}\right)+\left(\bar{\phi} \frac{\bar{G}^{j k} \bar{N}_{j} \bar{N}_{k}}{2 \bar{N}}\right) \partial_{t} \bar{\xi}^{t} \\
& +\left(\bar{\phi} \frac{\bar{N}_{k}}{\bar{N}}\right) \partial_{t} \bar{\xi}^{k}+\left(2-z+\Delta_{\phi}\right) \bar{\sigma}\left(\bar{\phi} \frac{\bar{G}^{j k} \bar{N}_{j} \bar{N}_{k}}{2 \bar{N}}\right), \\
\delta\left(\bar{\phi} \frac{\bar{N}_{i}}{\bar{N}}\right)= & \bar{\xi}^{\mu} \partial_{\mu}\left(\bar{\phi} \frac{\bar{N}_{i}}{\bar{N}^{\prime}}\right)+\left(\bar{\phi} \frac{\bar{N}_{k}}{\bar{N}}\right) \partial_{i} \bar{\xi}^{k} \\
& +\frac{\bar{\phi}}{\bar{N}} \bar{G}_{i k} \partial_{t} \bar{\xi}^{k}+\left(2-z+\Delta_{\phi}\right) \bar{\sigma}\left(\bar{\phi} \frac{\bar{N}_{i}}{\bar{N})} .\right.
\end{aligned}
$$

Here $\bar{\varepsilon}^{i j}$ is the Levi-Civita tensor associated with the boundary metric $\bar{G}_{i j}$.

\section{Calculating the gravitational Chern-Simons term}

In this appendix, we add a "-" to an index to denote the corresponding tangent space (vielbein frame) index. We extend the tangent space to 4 dimensions to include the temporal direction as well. 


\section{C.1 Bulk spin connection}

We define the vielbein 1 -form in the bulk as:

$$
\begin{aligned}
E^{\bar{t}} & =N \mathrm{~d} t, \\
E^{\bar{i}} & =\frac{L}{r} e_{i}^{\bar{i}}\left(N^{i} \mathrm{~d} t+\mathrm{d} x^{i}\right), \\
E^{\bar{r}} & =\frac{L}{r}\left(N^{r} \mathrm{~d} t+\mathrm{d} r\right),
\end{aligned}
$$

where $\bar{H}_{i j} \equiv \delta_{\overline{i j}} e_{i}^{\bar{i}} e_{j}^{\bar{j}}$ and $\bar{H}^{i j}$ is the matrix inverse of $\bar{H}_{i j}$. This is compatible with the gauge condition (3.11)-(3.13), with $\Upsilon(r)=L / r$. So far we have no identified $e_{i}^{\bar{i}}$ with the boundary vielbein $e_{i}^{a}$. It is a bulk quantity and a function of $r$ in general. $\bar{\varepsilon}_{i j}$ is the LeviCivita tensor defined via $\bar{H}_{i j}$. In this section we defined the spatial covariant derivative $\nabla_{i}$ is the one compatible with the spatial metric $\bar{H}_{i j}: \nabla_{k} \bar{H}_{i j}=0$ and the indices involved in $\nabla_{i}$ only run through $x$ and $y$. The spin connection 1 -form $\Omega^{\bar{M} \bar{N}}=\Omega_{\bar{M}}^{\bar{M} \bar{N}} \mathrm{~d} x^{M}$ is defined as

$$
\mathrm{d} E^{\bar{M}}+\Omega_{\bar{N}}^{\bar{M}} \wedge E^{\bar{N}}=0, \quad \Omega^{\bar{M} \bar{N}}=-\Omega^{\bar{N} \bar{M}} .
$$

Components of the spin connection can be computed using these two equations. The results are

$$
\begin{aligned}
\Omega_{t}^{\overline{t i}}= & -\frac{1}{2 N}\left(\frac{L}{r}\right)\left[e_{i}^{\bar{i}} N^{j} \nabla_{j} N^{i}+\bar{H}_{j k} N^{k} e^{\bar{i} i} \nabla_{i} N^{j}+N^{r} e^{\bar{i} i} \partial_{r}\left(\bar{H}_{i j} N^{j}\right)+N^{r} e^{\bar{i} i} \partial_{i} N^{r}\right. \\
& \left.-e^{\bar{i} i} N^{j} \partial_{t} \bar{H}_{i j}-\frac{2}{r} N^{r} e_{i}^{\bar{i}} N^{i}\right]+\frac{r}{L} e^{\bar{i} i} \partial_{i} N \\
\Omega_{j}^{\overline{t i}}= & -\frac{1}{2 N}\left(\frac{L}{r}\right)\left[e_{i}^{\bar{i}} \nabla_{j} N^{i}+e^{\bar{i} i} \nabla_{i}\left(\bar{H}_{j k} N^{k}\right)+N^{r} e^{\bar{i} i} \partial_{r} \bar{H}_{i j}-e^{\bar{i} i} \partial_{t} \bar{H}_{i j}-\frac{2}{r} N^{r} e_{j}^{\bar{i}}\right] \\
\Omega_{r}^{\overline{t i}}= & -\frac{1}{2 N}\left(\frac{L}{r}\right)\left[e_{i}^{\bar{i}} \partial_{r} N^{i}+e^{\bar{i} i} \partial_{i} N^{r}\right] \\
\Omega_{t}^{\bar{t} \bar{r}}= & -\frac{1}{2 N}\left(\frac{L}{r}\right)\left[\bar{H}_{i j} N^{i} \partial_{r} N^{j}+N^{i} \partial_{i} N^{r}+2 N^{r} \partial_{r} N^{r}-\frac{2}{r}\left(N^{r}\right)^{2}\right]+\frac{r}{L} \partial_{r} N \\
\Omega_{i}^{\bar{t} \bar{r}}= & -\frac{1}{2 N}\left(\frac{L}{r}\right)\left[\bar{H}_{i j} \partial_{r} N^{j}+\partial_{i} N^{r}\right] \\
\Omega_{r}^{\overline{t r}}= & -\frac{1}{N} \partial_{r}\left(\frac{L}{r} N^{r}\right), \\
\Omega_{t}^{\bar{i} \bar{j}}= & e^{\bar{i} k} \partial_{t} e_{k}^{\bar{j}}-\frac{1}{2} e^{\bar{i} i} e^{\bar{j} j} \partial_{t} \bar{H}_{i j}+\frac{1}{2} e^{\bar{i} i} e^{\bar{j} j}\left(\bar{H}_{i k} \nabla_{j} N^{k}-\bar{H}_{j k} \nabla_{i} N^{k}\right) \\
\Omega_{k}^{\bar{i} \bar{j}}= & e^{\bar{i} l} \nabla_{k} e_{l}^{\bar{j}}, \\
\Omega_{r}^{\bar{i} \bar{j}}= & e^{\bar{i} k} \partial_{r} e_{k}^{\bar{j}}-\frac{1}{2} e^{\bar{i} i} e^{\bar{j} j} \partial_{r} \bar{H}_{i j}=-e^{\bar{j} k} \partial_{r} e_{k}^{\bar{i}}+\frac{1}{2} e^{\bar{i} i} e^{\bar{j} j} \partial_{r} \bar{H}_{i j} \\
\Omega_{t}^{\bar{i} \bar{r}}= & \frac{1}{2} e_{i}^{\bar{i}} \partial_{r} N^{i}-\frac{1}{2} e^{\bar{i} i} \partial_{i} N^{r}+\frac{1}{2} e^{\bar{i} i} N^{j} \partial_{r} \bar{H}_{i j}-\frac{1}{r} e_{i}^{\bar{i}} N^{i} \\
\Omega_{j}^{\bar{i} \bar{r}}= & \frac{1}{2} e^{\bar{i} i} \partial_{r} \bar{H}_{i j}-\frac{1}{r} e_{j}^{\bar{i}}, \\
\Omega_{r}^{\bar{i} \bar{r}}= & 0
\end{aligned}
$$


Using $\Omega_{M}^{\bar{i} \bar{j}}=\frac{1}{2} \epsilon^{\bar{i} \bar{j}}\left(\epsilon_{\bar{k} \bar{l}} \Omega_{M}^{\bar{k} \bar{l}}\right), \Omega_{M}^{\bar{i} \bar{j}}$ can also be written as

$$
\begin{aligned}
& \Omega_{t}^{\bar{i} \bar{j}}=\epsilon^{\bar{i} \bar{j}}\left(\frac{1}{2} \epsilon_{\bar{k} \bar{l}} e^{\bar{k} j} \partial_{t} e_{j}^{\bar{l}}-\frac{1}{2} \bar{\varepsilon}^{k l} \bar{H}_{j l} \nabla_{k} N^{j}\right), \\
& \Omega_{k}^{\overline{i j}}=\epsilon^{\bar{i} \bar{j}}\left(\frac{1}{2} \epsilon_{\bar{k} \bar{l}} e^{\bar{k} j} \nabla_{k} e_{j}^{\bar{l}}\right)=\epsilon^{\bar{i} \bar{j}}\left(\frac{1}{2} \epsilon_{\bar{k} \bar{l}} e^{\bar{k} j} \partial_{k} e_{j}^{\bar{l}}-\frac{1}{2} \bar{\varepsilon}^{j l} \partial_{j} \bar{H}_{l k}\right), \\
& \Omega_{r}^{\bar{i} \bar{j}}=\epsilon^{\bar{i} \bar{j}}\left(\frac{1}{2} \epsilon_{\bar{k} \bar{l}} e^{\bar{k} j} \partial_{r} e_{j}^{\bar{l}}\right) .
\end{aligned}
$$

\section{C.2 Pontryagin density in the bulk}

The gravitational Chern-Simons term:

$$
S_{\mathrm{PY}}=\frac{c_{g}}{192 \pi} \int d^{4} x \sqrt{-G^{(4)}}\left({ }^{*} R R\right)
$$

where the Pontryagin density is

$$
{ }^{*} R R={ }^{*} R^{M N P Q} R_{N M P Q}, \quad{ }^{*} R^{M N P Q}=\frac{1}{2} \varepsilon^{P Q R S} R^{M N}{ }_{R S} .
$$

Here $R_{M N P Q}$ and $G^{(4)}$ are the Riemann tensor and determinant constructed from the full 4-dimensional metric. The curvature 2 -form is defined as

$$
R_{\bar{N}}^{\bar{M}}=\mathrm{d} \Omega_{\bar{N}}^{\bar{M}}+\Omega_{\bar{P}}^{\bar{M}} \wedge \Omega_{\bar{N}}^{\bar{P}}
$$

The Pontryagin current 3-form is defined as

$$
\mathcal{P}=\Omega^{\bar{M}}{ }_{\bar{N}} \wedge \mathrm{d} \Omega^{\bar{N}}{ }_{\bar{M}}+\frac{2}{3} \Omega^{\bar{M}}{ }_{\bar{N}} \wedge{\Omega^{\bar{N}}}_{\bar{P}} \wedge \Omega^{\bar{P}}{ }_{\bar{M}}=\mathcal{P}_{i j} \mathrm{~d} t \wedge \mathrm{d} x^{i} \wedge \mathrm{d} x^{j}+\ldots \wedge \mathrm{d} r,
$$

where the part denoted by ... is not relevant to our calculation, so we will keep denoting it as ... in the following. Taking exterior derivative on this equation, we have

$$
\mathrm{d} \mathcal{P}=\left\{-\partial_{r}\left(\epsilon^{i j} \mathcal{P}_{i j}\right)+\partial_{\mu}(\ldots)\right\} \mathrm{d} t \wedge \mathrm{d} x \wedge \mathrm{d} y \wedge \mathrm{d} r .
$$

On the other hand the Pontryagin density can be expressed as

$$
\mathrm{d} \mathcal{P}=\frac{1}{2} \sqrt{-G^{(4)}}\left({ }^{*} R R\right) \mathrm{d} t \wedge \mathrm{d} x \wedge \mathrm{d} y \wedge \mathrm{d} r .
$$

Thus we arrive at

$$
\sqrt{-G^{(4)}}{ }^{*} R R=-2 \partial_{r}\left(\epsilon^{i j} \mathcal{P}_{i j}\right)+\partial_{\mu}(\ldots) .
$$

This shows that the gravitational Chern-Simons term is a boundary term as well:

$$
S_{\mathrm{CS}}^{g}=\frac{c_{g}}{96 \pi} \int_{r=0} d^{3} x \sqrt{\bar{H}} \bar{\varepsilon}^{i j} \overline{\mathcal{P}}_{i j}
$$

where $\overline{\mathcal{P}}_{i j}$ is the boundary limit of $\mathcal{P}_{i j}$ and it will be computed explicitly in the next subsection. 


\section{C.3 Pontryagin current at the boundary}

We now compute $\overline{\mathcal{P}}_{i j}$ at the boundary. Using the near-boundary behaviors of the bulk fields:

$$
N \Rightarrow\left(\frac{L}{r}\right)^{z} \bar{N}, \quad N^{I} \Rightarrow\left(\frac{L}{r}\right)^{0} \bar{N}^{I}, \quad \bar{H}_{i j} \Rightarrow \bar{G}_{i j}
$$

the condition $\bar{N}_{r}=0$, and identify $e_{i}^{\bar{i}}$ at the boundary with the boundary vielbein, the near-boundary behavior of the spin connection is

$$
\begin{aligned}
\Omega^{\overline{t i}} & \Rightarrow\left(\frac{L}{r}\right)^{1-z} \alpha_{i}^{\bar{i}}\left(\mathrm{~d} x^{i}+\bar{N}^{i} \mathrm{~d} t\right)+\left(\frac{L}{r}\right)^{z-1}\left(e^{\bar{i} i} \partial_{i} \bar{N}\right) \mathrm{d} t+\ldots \mathrm{d} r \\
\Omega^{\bar{t} \bar{r}} & \Rightarrow\left(\frac{L}{r}\right)^{1-z} \beta_{i}\left(\mathrm{~d} x^{i}+\bar{N}^{i} \mathrm{~d} t\right)+\left(\frac{L}{r}\right)^{z-1}\left(\partial_{r} \bar{N}\right) \mathrm{d} t \\
\Omega^{\bar{i} \bar{r}} & \Rightarrow \gamma_{i}^{\bar{i}}\left(\mathrm{~d} x^{i}+\bar{N}^{i} \mathrm{~d} t\right)+\left(\frac{1}{2} e_{i}^{\bar{i}} \partial_{r} \bar{N}^{i}\right) \mathrm{d} t, \\
\Omega^{\bar{i} \bar{j}} & \Rightarrow \frac{1}{2} \epsilon^{\bar{i} \bar{j}}\left(\Omega_{t} \mathrm{~d} t+\Omega_{k} \mathrm{~d} x^{k}\right)+\ldots \mathrm{d} r
\end{aligned}
$$

where

$$
\begin{aligned}
\alpha_{i}^{\bar{i}} & =-\frac{1}{2 \bar{N}} e^{\overline{i j}}\left(\nabla_{i} \bar{N}_{j}+\nabla_{j} \bar{N}_{i}-\partial_{t} \bar{G}_{i j}\right), \\
\beta_{i} & =-\frac{1}{2 \bar{N}} \bar{G}_{i j} \partial_{r} \bar{N}^{j}, \\
\gamma_{i}^{\bar{i}} & =\frac{1}{2} e^{\overline{i j} j} \partial_{r} \bar{G}_{i j}-\frac{1}{r} e_{i}^{\bar{i}}, \\
\Omega_{t} & =\epsilon_{\bar{i} \bar{j}} e^{\bar{i} l} \partial_{t} e_{l}^{\bar{j}}-\bar{\varepsilon}^{i j} \partial_{i} \bar{N}_{j}, \\
\Omega_{k} & =\epsilon_{\overline{i j}} e^{\bar{i} l} \nabla_{k} e_{l}^{\bar{j}}=\epsilon_{\overline{i j}} e^{\bar{i} l} \partial_{k} e_{l}^{\bar{j}}-\bar{\varepsilon}^{i j} \partial_{i} \bar{G}_{j k},
\end{aligned}
$$

and now the spatial covariant derivative $\nabla_{i}$ is the one compatible with the spatial metric $\bar{G}_{i j}: \nabla_{k} \bar{G}_{i j}=0$ and the indices involved in $\nabla_{i}$ only run through $x$ and $y$. Then the relevant term in the Pontryagin current is given by

$$
\mathcal{P}_{i j} \Rightarrow\left(\frac{r}{L}\right)^{2(z-1)} \overline{\mathcal{P}}_{i j}^{\mathrm{sig}}+\overline{\mathcal{P}}_{i j}^{\mathrm{reg}}
$$

where

$$
\begin{aligned}
\overline{\mathcal{P}}_{i j}^{\text {sig }}= & -\alpha_{i}^{\bar{i}}\left(\partial_{t} \alpha_{j}^{\bar{i}}\right)+\alpha_{i}^{\bar{i}} \partial_{j}\left(\alpha_{k}^{\bar{i}} \bar{N}^{k}\right)-\alpha_{k}^{\bar{i}} \bar{N}^{k}\left(\partial_{j} \alpha_{i}^{\bar{i}}\right)-\frac{1}{2} \epsilon_{i \bar{j}} \alpha_{i}^{\bar{i}} \alpha_{j}^{\bar{j}} \Omega_{t}+\epsilon_{i \bar{j}} \alpha_{i}^{\bar{i}} \alpha_{k}^{\bar{j}} \bar{N}^{k} \Omega_{j} \\
& -\beta_{i}\left(\partial_{t} \beta_{j}\right)+\beta_{i} \partial_{j}\left(\beta_{k} \bar{N}^{k}\right)-\beta_{k} \bar{N}^{k}\left(\partial_{j} \beta_{i}\right)-2 \alpha_{i}^{\bar{i}} \beta_{j}\left(\gamma_{k}^{\bar{i}} \bar{N}^{k}+\frac{1}{2} e_{k}^{\bar{i}} \partial_{r} \bar{N}^{k}\right) \\
& +2 \alpha_{k}^{\bar{i}} \bar{N}^{k} \beta_{j} \gamma_{i}^{\bar{i}}+2 \beta_{k} \bar{N}^{k} \alpha_{i}^{\bar{i}} \gamma_{j}^{\bar{i}}-(i \leftrightarrow j),
\end{aligned}
$$




$$
\begin{aligned}
\overline{\mathcal{P}}_{i j}^{\mathrm{reg}}= & {\left[\alpha_{i}^{\bar{i}}\left(\partial_{j} e^{\bar{i} k}\right)-e^{\bar{i} k}\left(\partial_{j} \alpha_{i}^{\bar{i}}\right)+\epsilon_{\overline{i j}} \alpha_{i}^{\bar{i}} e^{\bar{j} k} \Omega_{j}+2 e^{\bar{i} k} \beta_{j} \gamma_{i}^{\bar{i}}\right]\left(\partial_{k} \bar{N}\right)+\alpha_{i}^{\bar{i}} e^{\bar{i} k}\left(\partial_{j} \partial_{k} \bar{N}\right) } \\
& +\left[-\gamma_{i}^{\bar{i}}\left(\partial_{j} \gamma_{k}^{\bar{i}}\right)+\gamma_{k}^{\bar{i}}\left(\partial_{j} \gamma_{i}^{\bar{i}}\right)-\epsilon_{\bar{i} \bar{j}} \gamma_{i}^{\bar{i}} \gamma_{k}^{\bar{j}} \Omega_{j}\right] \bar{N}^{k}-\gamma_{i}^{\bar{i}} \gamma_{k}^{\bar{i}}\left(\partial_{j} \bar{N}^{k}\right)+\gamma_{i}^{\bar{i}}\left(\partial_{t} \gamma_{j}^{\bar{i}}\right) \\
& +\frac{1}{2} \epsilon_{\bar{i} \bar{j}} \gamma_{i}^{\bar{i}} \gamma_{j}^{\bar{j}} \Omega_{t}-\frac{1}{4}\left[\Omega_{t}\left(\partial_{i} \Omega_{j}\right)+\Omega_{i}\left(\partial_{j} \Omega_{t}-\partial_{t} \Omega_{j}\right)\right]+\beta_{i}\left(\partial_{j} \partial_{r} \bar{N}\right) \\
& -\frac{1}{2} \gamma_{i}^{\bar{i}} e_{k}^{\bar{i}}\left(\partial_{j} \partial_{r} \bar{N}^{k}\right)+\frac{1}{2}\left[-\gamma_{i}^{\bar{i}}\left(\partial_{j} e_{k}^{\bar{i}}\right)+e_{k}^{\bar{i}}\left(\partial_{j} \gamma_{i}^{\bar{i}}\right)-\epsilon_{\bar{i} \bar{j}} \gamma_{i}^{\bar{i}} e_{k}^{\bar{j}} \Omega_{j}\right]\left(\partial_{r} \bar{N}^{k}\right) \\
& +\left(2 \alpha_{i}^{\bar{i}} \gamma_{j}^{\bar{i}}-\partial_{j} \beta_{i}\right)\left(\partial_{r} \bar{N}\right)-(i \leftrightarrow j),
\end{aligned}
$$

and $\bar{i}$ index in terms such as $\alpha_{i}^{\bar{i}}\left(\partial_{t} \alpha_{j}^{\bar{i}}\right)$ are understood to be summed over, which is the same as $\delta_{\overline{i j}} \alpha_{i}^{\bar{i}}\left(\partial_{t} \alpha_{j}^{\bar{j}}\right)$.

There are simplifications that can dramatically reduce the number of terms in the above expressions. First, we assume $z>1$, then $\overline{\mathcal{P}}_{i j}^{\text {sig }}$ completely drops off near the boundary $r \rightarrow 0$. This can also be understood by thinking of the non-relativistic limit $c \rightarrow \infty$. We can restore the dependence of speed of light $c$ by just replacing $N$ by $c N$, hence $\bar{N}$ by $c \bar{N}$ in the above expressions. Both $\alpha_{i}^{\bar{i}}$ and $\beta_{i}$ are proportional to $\bar{N}^{-1}$, thus are of order $O\left(c^{-1}\right)$. Every term in $\overline{\mathcal{P}}_{i j}^{\text {sig }}$ is either quadratic in $\alpha_{i}^{\bar{i}}$ or $\beta_{i}$, or proportional to a product of them. Thus we have

$$
\overline{\mathcal{P}}_{i j}^{\text {sig }} \sim O\left(\frac{1}{c^{2}}\right), \quad \overline{\mathcal{P}}_{i j}=\overline{\mathcal{P}}_{i j}^{\text {reg }}+O\left(\frac{1}{c^{2}}\right) .
$$

Of course when $z=1$, corresponding to the Lorentzian case, it gives a finite contribution, but for simplicity, we will not consider this case here. The second simplification comes from adding the Gibbons-Hawking term (4.34). As we have argued before, it completely removes all $\partial_{r}$ terms without changing any other term. Thus all $\partial_{r}$ terms in $\overline{\mathcal{P}}_{i j}^{\text {reg }}$, including all terms containing $\beta_{i}$ and $\gamma_{i}^{\bar{i}}$, drop off. ${ }^{19}$ By using the identity

$$
\epsilon_{\overline{i j}} e^{\bar{i} l} \nabla_{k} e_{l}^{\bar{j}}=-\bar{\varepsilon}_{j l} e^{\bar{i} j} \nabla_{k} e^{\bar{i} l}
$$

the first line in the expression of $\overline{\mathcal{P}}_{i j}^{\text {reg }}$ can also be simplified. At the end, we have

$$
\begin{aligned}
\bar{\varepsilon}^{i j} \overline{\mathcal{P}}_{i j}= & \bar{\varepsilon}^{i j} \bar{G}^{k l}\left\{\left(\nabla_{k} \bar{N}\right) \nabla_{j}\left[\frac{1}{\bar{N}}\left(\nabla_{i} \bar{N}_{l}+\nabla_{l} \bar{N}_{i}-\partial_{t} \bar{G}_{i l}\right)\right]\right. \\
& \left.-\left(\nabla_{j} \nabla_{k} \bar{N}\right) \frac{1}{\bar{N}}\left(\nabla_{i} \bar{N}_{l}+\nabla_{l} \bar{N}_{i}-\partial_{t} \bar{G}_{i l}\right)\right\} \\
& -\frac{1}{2} \bar{\varepsilon}^{i j}\left[\Omega_{t} \partial_{i} \Omega_{j}+\Omega_{i}\left(\partial_{j} \Omega_{t}-\partial_{t} \Omega_{j}\right)\right]+O\left(\partial_{r}\right),
\end{aligned}
$$

for $z>1$.

\footnotetext{
${ }^{19}$ The $\frac{1}{r} e_{i}^{\bar{i}}$ term in $\gamma_{i}^{\bar{i}}$ is a consequence of $\partial_{r}$ hitting $\frac{L}{r}$, thus is part of the $\partial_{r}$ terms that are removed by the Gibbons-Hawking term.
} 
Open Access. This article is distributed under the terms of the Creative Commons Attribution License (CC-BY 4.0), which permits any use, distribution and reproduction in any medium, provided the original author(s) and source are credited.

\section{References}

[1] D.C. Tsui, H.L. Stormer and A.C. Gossard, Two-dimensional magnetotransport in the extreme quantum limit, Phys. Rev. Lett. 48 (1982) 1559 [INSPIRE].

[2] R.B. Laughlin, Anomalous quantum Hall effect: An Incompressible quantum fluid with fractionallycharged excitations, Phys. Rev. Lett. 50 (1983) 1395 [INSPIRE].

[3] S.C. Zhang, T.H. Hansson and S. Kivelson, An effective field theory model for the fractional quantum hall effect, Phys. Rev. Lett. 62 (1988) 82 [INSPIRE].

[4] E. Fradkin and A. Lopez, Fractional Quantum Hall effect and Chern-Simons gauge theories, Phys. Rev. B 44 (1991) 5246 [InSPIRE].

[5] X.G. Wen and A. Zee, A Classification of Abelian quantum Hall states and matrix formulation of topological fluids, Phys. Rev. B 46 (1992) 2290 [INSPIRE].

[6] S.-C. Zhang, The Chern-Simons-Landau-Ginzburg theory of the fractional quantum Hall effect, Int. J. Mod. Phys. B 6 (1992) 25 [INSPIRE].

[7] B.I. Halperin, P.A. Lee and N. Read, Theory of the half filled Landau level, Phys. Rev. B 47 (1993) 7312 [INSPIRE].

[8] R.B. Laughlin, Quantized Hall conductivity in two-dimensions, Phys. Rev. B 23 (1981) 5632 [INSPIRE].

[9] D.J. Thouless, M. Kohmoto, M.P. Nightingale and M. den Nijs, Quantized Hall Conductance in a Two-Dimensional Periodic Potential, Phys. Rev. Lett. 49 (1982) 405 [INSPIRE].

[10] Q. Niu, D.J. Thouless and Y.-S. Wu, Quantized Hall conductance as a topological invariant, Phys. Rev. B 31 (1985) 3372 [INSPIRE].

[11] B.I. Halperin, Statistics of quasiparticles and the hierarchy of fractional quantized Hall states, Phys. Rev. Lett. 52 (1984) 1583 [Erratum ibid. 52 (1984) 2390] [INSPIRE].

[12] D. Arovas, J.R. Schrieffer and F. Wilczek, Fractional Statistics and the Quantum Hall Effect, Phys. Rev. Lett. 53 (1984) 722 [InSPIRE].

[13] X.G. Wen, Gapless Boundary Excitations in the Quantum Hall States and in the Chiral Spin States, Phys. Rev. B 43 (1991) 11025 [INSPIRE].

[14] X.G. Wen, Electrodynamical Properties of Gapless Edge Excitations in the Fractional Quantum Hall States, Phys. Rev. Lett. 64 (1990) 2206 [INSPIRE].

[15] X.G. Wen, Theory of the edge states in fractional quantum Hall effects, Int. J. Mod. Phys. B 6 (1992) 1711 [INSPIRE].

[16] J. Fröhlich and T. Kerler, Universality in quantum Hall systems, Nucl. Phys. B 354 (1991) 369 [INSPIRE].

[17] J. Fröhlich and A. Zee, Large scale physics of the quantum Hall fluid, Nucl. Phys. B 364 (1991) 517 [INSPIRE]. 
[18] F.D.M. Haldane, Geometrical Description of the Fractional Quantum Hall Effect, Phys. Rev. Lett. 107 (2011) 116801 [arXiv:1106.3375] [INSPIRE].

[19] K. von Klitzing, G. Dorda and M. Pepper, New method for high accuracy determination of the fine structure constant based on quantized Hall resistance, Phys. Rev. Lett. 45 (1980) 494 [INSPIRE].

[20] S.M. Girvin and T. Jach, Formalism for the quantum Hall effect: Hilbert space of analytic functions, Phys. Rev. B 29 (1984) 5617.

[21] G.V. Dunne, Hilbert space for charged particles in perpendicular magnetic fields, Annals Phys. 215 (1992) 233 [INSPIRE].

[22] A. Cappelli, C.A. Trugenberger and G.R. Zemba, Infinite symmetry in the quantum Hall effect, Nucl. Phys. B 396 (1993) 465 [hep-th/9206027] [InSPIRE].

[23] S.M. Girvin, A.H. MacDonald and P.M. Platzman, Magneto-roton theory of collective excitations in the fractional quantum Hall effect, Phys. Rev. B 33 (1986) 2481 [InSPIRE].

[24] X.G. Wen and A. Zee, Shift and spin vector: New topological quantum numbers for the Hall fluids, Phys. Rev. Lett. 69 (1992) 953 [Erratum ibid. 69 (1992) 3000] [INSPIRE].

[25] J.E. Avron, R. Seiler and P.G. Zograf, Viscosity of quantum Hall fluids, Phys. Rev. Lett. 75 (1995) 697 [INSPIRE].

[26] J.E. Avron, Odd Viscosity, J. Stat. Phys. 92 (1998) 543 [physics/9712050].

[27] I.V. Tokatly and G. Vignale, Lorentz shear modulus of a two-dimensional electron gas at high magnetic field, Phys. Rev. B 76 (2007) 161305 [arXiv:0706.2454].

[28] I.V. Tokatly and G. Vignale, Lorentz shear modulus of fractional quantum Hall states, Journal of Phys. Condens. Matter 21 (2009) 275603 [arXiv:0812.4331].

[29] N. Read, Non-Abelian adiabatic statistics and Hall viscosity in quantum Hall states and $p_{x}+i p_{y}$ paired superfluids, Phys. Rev. B 79 (2009) 045308 [arXiv:0805.2507] [INSPIRE].

[30] N. Read and E.H. Rezayi, Hall viscosity, orbital spin and geometry: paired superfluids and quantum Hall systems, Phys. Rev. B 84 (2011) 085316 [arXiv: 1008.0210] [InSPIRE].

[31] C. Hoyos and D.T. Son, Hall Viscosity and Electromagnetic Response, Phys. Rev. Lett. 108 (2012) 066805 [arXiv:1109.2651] [inSPIRE].

[32] Y. Hidaka, Y. Hirono, T. Kimura and Y. Minami, Viscoelastic-electromagnetism and Hall viscosity, PTEP 2013 (2013) 013A02 [arXiv:1206.0734] [INSPIRE].

[33] P. Wiegmann, Anomalous Hydrodynamics of Fractional Quantum Hall States, JETP 117 (2013) 538 [arXiv: 1305.6893].

[34] R.R. Biswas, Semiclassical theory of viscosity in quantum Hall states, arXiv:1311.7149 [INSPIRE].

[35] M. Fremling, T.H. Hansson and J. Suorsa, Hall Viscosity of Hierarchical Quantum Hall States, Phys. Rev. B 89 (2014) 125303 [arXiv:1312.6038] [InSPIRE].

[36] C. Hoyos, Hall viscosity, topological states and effective theories, Int. J. Mod. Phys. B 28 (2014) 1430007 [arXiv:1403.4739] [INSPIRE].

[37] C. Hoyos, S. Moroz and D.T. Son, Effective theory of chiral two-dimensional superfluids, Phys. Rev. B 89 (2014) 174507 [arXiv:1305.3925] [InSPIRE]. 
[38] S. Moroz and C. Hoyos, Effective theory of two-dimensional chiral superfluids: gauge duality and Newton-Cartan formulation, arXiv:1408.5911 [INSPIRE].

[39] A. Shitade and T. Kimura, Bulk angular momentum and Hall viscosity in chiral superconductors, Phys. Rev. B 90 (2014) 134510 [arXiv:1407.1877] [INSPIRE].

[40] C. Hoyos, B.S. Kim and Y. Oz, Odd Parity Transport In Non-Abelian Superfluids From Symmetry Locking, JHEP 10 (2014) 127 [arXiv:1404.7507] [INSPIRE].

[41] T.L. Hughes, R.G. Leigh and E. Fradkin, Torsional Response and Dissipationless Viscosity in Topological Insulators, Phys. Rev. Lett. 107 (2011) 075502 [arXiv:1101.3541] [INSPIRE].

[42] T.L. Hughes, R.G. Leigh and O. Parrikar, Torsional Anomalies, Hall Viscosity and Bulk-boundary Correspondence in Topological States, Phys. Rev. D 88 (2013) 025040 [arXiv: 1211.6442] [INSPIRE].

[43] O. Parrikar, T.L. Hughes and R.G. Leigh, Torsion, parity-odd response and anomalies in topological states, Phys. Rev. D 90 (2014) 105004 [arXiv: 1407.7043] [INSPIRE].

[44] M. Geracie and D.T. Son, Effective field theory for fluids: Hall viscosity from a Wess-Zumino-Witten term, JHEP 11 (2014) 004 [arXiv: 1402.1146] [INSPIRE].

[45] S. Golkar, M.M. Roberts and D.T. Son, Effective Field Theory of Relativistic Quantum Hall Systems, JHEP 12 (2014) 138 [arXiv:1403.4279] [INSPIRE].

[46] S. Golkar, M.M. Roberts and D.T. Son, The Euler current and relativistic parity odd transport, arXiv:1407.7540 [inSPIRE].

[47] A. Nicolis and D.T. Son, Hall viscosity from effective field theory, arXiv:1103.2137 [INSPIRE].

[48] B. Bradlyn, M. Goldstein and N. Read, Kubo formulas for viscosity: Hall viscosity, Ward identities and the relation with conductivity, Phys. Rev. B 86 (2012) 245309 [arXiv:1207.7021] [INSPIRE].

[49] C. Hoyos, B.S. Kim and Y. Oz, Ward Identities for Hall Transport, arXiv:1407.2616 [INSPIRE].

[50] F.D.M. Haldane, 'Hall viscosity' and intrinsic metric of incompressible fractional Hall fluids, arXiv:0906.1854 [INSPIRE].

[51] F.D.M. Haldane, Self-duality and long-wavelength behavior of the Landau-level guiding-center structure function, and the shear modulus of fractional quantum Hall fluids, arXiv:1112.0990.

[52] Y. Park and F.D.M. Haldane, Guiding-center Hall viscosity and intrinsic dipole moment along edges of incompressible fractional quantum Hall fluids, Phys. Rev. B 90 (2014) 045123 [arXiv: 1403.2822].

[53] B. Yang, Z.X. Hu, Z. Papic and F.D.M. Haldane, Model Wave Functions for the Collective Modes and the Magnetoroton Theory of the Fractional Quantum Hall Effect, Phys. Rev. Lett. 108 (2012) 256807 [arXiv:1201.4165].

[54] M. Mulligan, C. Nayak and S. Kachru, An Isotropic to Anisotropic Transition in a Fractional Quantum Hall State, Phys. Rev. B 82 (2010) 085102 [arXiv:1004.3570] [INSPIRE].

[55] M. Mulligan, C. Nayak and S. Kachru, Effective Field Theory of Fractional Quantized Hall Nematics, Phys. Rev. B 84 (2011) 195124 [arXiv:1104.0256] [INSPIRE]. 
[56] J. Maciejko, B. Hsu, S.A. Kivelson, Y. Park and S.L. Sondhi, Field theory of the quantum Hall nematic transition, Phys. Rev. B 88 (2013) 125137 [arXiv: 1303.3041] [INSPIRE].

[57] Y. You and E. Fradkin, Field Theory of Nematicity in the Spontaneous Quantum Anomalous Hall effect, Phys. Rev. B 88 (2013) 235124 [arXiv:1310.5727] [InSPIRE].

[58] J. Fröhlich and U.M. Studer, Gauge invariance and current algebra in nonrelativistic many body theory, Rev. Mod. Phys. 65 (1993) 733 [inSPIRE].

[59] D.T. Son and M. Wingate, General coordinate invariance and conformal invariance in nonrelativistic physics: Unitary Fermi gas, Annals Phys. 321 (2006) 197 [cond-mat/0509786] [INSPIRE].

[60] M. Geracie, D.T. Son, C. Wu and S.-F. Wu, Spacetime Symmetries of the Quantum Hall Effect, arXiv:1407.1252 [INSPIRE].

[61] D.T. Son, Newton-Cartan geometry and the quantum Hall effect, arXiv:1306.0638 [INSPIRE].

[62] S. Golkar, D.X. Nguyen and D.T. Son, Spectral Sum Rules and Magneto-Roton as Emergent Graviton in Fractional Quantum Hall Effect, arXiv:1309.2638 [INSPIRE].

[63] M. Geracie and D.T. Son, Hydrodynamics on the lowest Landau level, arXiv:1408.6843 [INSPIRE].

[64] A. Gromov and A.G. Abanov, Thermal Hall Effect and Geometry with Torsion, Phys. Rev. Lett. 113 (2014) 266802 [arXiv: 1407.2908] [INSPIRE].

[65] K. Jensen, On the coupling of Galilean-invariant field theories to curved spacetime, arXiv: 1408.6855 [INSPIRE].

[66] O. Andreev, M. Haack and S. Hofmann, On Nonrelativistic Diffeomorphism Invariance, Phys. Rev. D 89 (2014) 064012 [arXiv:1309.7231] [INSPIRE].

[67] A.G. Abanov and A. Gromov, Electromagnetic and gravitational responses of two-dimensional non-interacting electrons in background magnetic field, Phys. Rev. B 90 (2014) 014435 [arXiv:1401.3703] [INSPIRE].

[68] A. Gromov and A.G. Abanov, Density-curvature response and gravitational anomaly, arXiv: 1403.5809 [INSPIRE].

[69] O. Andreev, More On Nonrelativistic Diffeomorphism Invariance, arXiv:1408.7031 [INSPIRE].

[70] R. Banerjee, A. Mitra and P. Mukherjee, A new formulation of non-relativistic diffeomorphism invariance, Phys. Lett. B 737 (2014) 369 [arXiv:1404.4491] [INSPIRE].

[71] R. Banerjee, A. Mitra and P. Mukherjee, Localisation of the Galilean symmetry and dynamical realisation of Newton-Cartan geometry, arXiv:1407.3617 [INSPIRE].

[72] T. Brauner, S. Endlich, A. Monin and R. Penco, General coordinate invariance in quantum many-body systems, Phys. Rev. D 90 (2014) 105016 [arXiv:1407.7730] [INSPIRE].

[73] B. Bradlyn and N. Read, Low-energy effective theory in the bulk for transport in a topological phase, arXiv:1407.2911 [INSPIRE].

[74] C. Duval and P.A. Horvathy, Non-relativistic conformal symmetries and Newton-Cartan structures, J. Phys. A 42 (2009) 465206 [arXiv:0904.0531] [InSPIRE]. 
[75] D. Blas, O. Pujolàs and S. Sibiryakov, On the extra mode and inconsistency of Hor̆ava gravity, JHEP 10 (2009) 029 [arXiv:0906.3046] [INSPIRE].

[76] C. Germani, A. Kehagias and K. Sfetsos, Relativistic quantum gravity at a Lifshitz point, JHEP 09 (2009) 060 [arXiv:0906.1201] [INSPIRE].

[77] J. Kluson, Note about non-relativistic diffeomorphism invariant gravity action in three dimensions, Phys. Rev. D 89 (2014) 104036 [arXiv:1401.7812] [INSPIRE].

[78] J.M. Maldacena, The Large- $N$ limit of superconformal field theories and supergravity, Int. J. Theor. Phys. 38 (1999) 1113 [hep-th/9711200] [INSPIRE].

[79] S.S. Gubser, I.R. Klebanov and A.M. Polyakov, Gauge theory correlators from noncritical string theory, Phys. Lett. B 428 (1998) 105 [hep-th/9802109] [INSPIRE].

[80] E. Witten, Anti-de Sitter space and holography, Adv. Theor. Math. Phys. 2 (1998) 253 [hep-th/9802150] [INSPIRE].

[81] G. Policastro, D.T. Son and A.O. Starinets, From AdS/CFT correspondence to hydrodynamics, JHEP 09 (2002) 043 [hep-th/0205052] [INSPIRE].

[82] P. Kovtun, D.T. Son and A.O. Starinets, Holography and hydrodynamics: diffusion on stretched horizons, JHEP 10 (2003) 064 [hep-th/0309213] [INSPIRE].

[83] A. Buchel and J.T. Liu, Universality of the shear viscosity in supergravity, Phys. Rev. Lett. 93 (2004) 090602 [hep-th/0311175] [INSPIRE].

[84] P. Kovtun, D.T. Son and A.O. Starinets, Viscosity in strongly interacting quantum field theories from black hole physics, Phys. Rev. Lett. 94 (2005) 111601 [hep-th/0405231] [INSPIRE].

[85] S.A. Hartnoll, Lectures on holographic methods for condensed matter physics, Class. Quant. Grav. 26 (2009) 224002 [arXiv: 0903.3246] [inSPIRE].

[86] C.P. Herzog, Lectures on Holographic Superfluidity and Superconductivity, J. Phys. A 42 (2009) 343001 [arXiv:0904.1975] [INSPIRE].

[87] J. McGreevy, Holographic duality with a view toward many-body physics, Adv. High Energy Phys. 2010 (2010) 723105 [arXiv:0909.0518] [INSPIRE].

[88] G.T. Horowitz, Introduction to Holographic Superconductors, Lect. Notes Phys. 828 (2011) 313 [arXiv: 1002.1722] [INSPIRE].

[89] S. Sachdev, Condensed Matter and AdS/CFT, Lect. Notes Phys. 828 (2011) 273 [arXiv: 1002 .2947] [INSPIRE].

[90] N. Iqbal, H. Liu and M. Mezei, Lectures on holographic non-Fermi liquids and quantum phase transitions, arXiv:1110.3814 [INSPIRE].

[91] A. Adams, L.D. Carr, T. Schäfer, P. Steinberg and J.E. Thomas, Strongly correlated quantum fluids: ultracold quantum gases, quantum chromodynamic plasmas and holographic duality, New J. Phys. 14 (2012) 115009 [arXiv:1205.5180] [INSPIRE].

[92] S. Kachru, X. Liu and M. Mulligan, Gravity duals of Lifshitz-like fixed points, Phys. Rev. D 78 (2008) 106005 [arXiv:0808.1725] [INSPIRE].

[93] M. Taylor, Non-relativistic holography, arXiv:0812.0530 [INSPIRE].

[94] M.H. Christensen, J. Hartong, N.A. Obers and B. Rollier, Torsional Newton-Cartan geometry and Lifshitz holography, Phys. Rev. D 89 (2014) 061901 [arXiv:1311.4794] [INSPIRE]. 
[95] M.H. Christensen, J. Hartong, N.A. Obers and B. Rollier, Boundary stress-energy tensor and Newton-Cartan geometry in Lifshitz holography, JHEP 01 (2014) 057 [arXiv:1311.6471] [INSPIRE].

[96] W. Chemissany and I. Papadimitriou, Generalized dilatation operator method for non-relativistic holography, Phys. Lett. B 737 (2014) 272 [arXiv:1405.3965] [INSPIRE].

[97] W. Chemissany and I. Papadimitriou, Lifshitz holography: the whole shebang, arXiv: 1408.0795 [INSPIRE].

[98] E. Keski-Vakkuri and P. Kraus, Quantum Hall effect in AdS/CFT, JHEP 09 (2008) 130 [arXiv: 0805.4643] [INSPIRE].

[99] J.L. Davis, P. Kraus and A. Shah, Gravity dual of a quantum Hall plateau transition, JHEP 11 (2008) 020 [arXiv:0809.1876] [INSPIRE].

[100] M. Fujita, W. Li, S. Ryu and T. Takayanagi, Fractional quantum Hall effect via holography: Chern-Simons, edge states and hierarchy, JHEP 06 (2009) 066 [arXiv:0901.0924] [INSPIRE].

[101] Y. Hikida, W. Li and T. Takayanagi, ABJM with flavors and FQHE, JHEP 07 (2009) 065 [arXiv:0903.2194] [INSPIRE].

[102] J. Alanen, E. Keski-Vakkuri, P. Kraus and V. Suur-Uski, AC transport at holographic quantum Hall transitions, JHEP 11 (2009) 014 [arXiv:0905.4538] [INSPIRE].

[103] O. Bergman, N. Jokela, G. Lifschytz and M. Lippert, Quantum Hall effect in a holographic model, JHEP 10 (2010) 063 [arXiv: 1003.4965] [INSPIRE].

[104] A. Bayntun, C.P. Burgess, B.P. Dolan and S.-S. Lee, AdS/QHE: towards a holographic description of quantum Hall experiments, New J. Phys. 13 (2011) 035012 [arXiv: 1008.1917] [INSPIRE].

[105] E. Gubankova, J. Brill, M. Cubrovic, K. Schalm, P. Schijven et al., Holographic fermions in external magnetic fields, Phys. Rev. D 84 (2011) 106003 [arXiv:1011.4051] [InSPIRE].

[106] N. Jokela, G. Lifschytz and M. Lippert, Magneto-roton excitation in a holographic quantum Hall fluid, JHEP 02 (2011) 104 [arXiv:1012.1230] [INSPIRE].

[107] M. Fujita, M. Kaminski and A. Karch, $\mathrm{SL}(2, \mathbb{Z})$ action on AdS/BCFT and Hall conductivities, JHEP 07 (2012) 150 [arXiv:1204.0012] [INSPIRE].

[108] D. Melnikov, E. Orazi and P. Sodano, On the AdS/BCFT approach to quantum Hall systems, JHEP 05 (2013) 116 [arXiv:1211.1416] [INSPIRE].

[109] C. Kristjansen and G.W. Semenoff, Giant D5 brane holographic Hall state, JHEP 06 (2013) 048 [arXiv: 1212.5609] [INSPIRE].

[110] N. Jokela, G. Lifschytz and M. Lippert, Holographic anyonic superfluidity, JHEP 10 (2013) 014 [arXiv: 1307.6336] [INSPIRE].

[111] C. Kristjansen, R. Pourhasan and G.W. Semenoff, A holographic quantum Hall ferromagnet, JHEP 02 (2014) 097 [arXiv: 1311.6999] [INSPIRE].

[112] O. Aharony, S.S. Gubser, J.M. Maldacena, H. Ooguri and Y. Oz, Large-N field theories, string theory and gravity, Phys. Rept. 323 (2000) 183 [hep-th/9905111] [INSPIRE].

[113] K. Balasubramanian and J. McGreevy, The Particle number in Galilean holography, JHEP 01 (2011) 137 [arXiv: 1007.2184] [INSPIRE]. 
[114] D.T. Son, Toward an AdS/cold atoms correspondence: a geometric realization of the Schrödinger symmetry, Phys. Rev. D 78 (2008) 046003 [arXiv:0804.3972] [INSPIRE].

[115] K. Balasubramanian and J. McGreevy, Gravity duals for non-relativistic CFTs, Phys. Rev. Lett. 101 (2008) 061601 [arXiv:0804.4053] [INSPIRE].

[116] V.K. Dobrev, Non-relativistic holography - A group-theoretical perspective, Int. J. Mod. Phys. A 29 (2014) 1430001 [arXiv:1312.0219] [INSPIRE].

[117] S. Janiszewski and A. Karch, Non-relativistic holography from Hor̆ava gravity, JHEP 02 (2013) 123 [arXiv:1211.0005] [InSPIRE].

[118] S. Janiszewski and A. Karch, String Theory Embeddings of Nonrelativistic Field Theories and Their Holographic Hořava Gravity Duals, Phys. Rev. Lett. 110 (2013) 081601 [arXiv: 1211.0010] [INSPIRE].

[119] P. Hořava, Membranes at quantum criticality, JHEP 03 (2009) 020 [arXiv:0812.4287] [INSPIRE].

[120] P. Hořava, Quantum gravity at a Lifshitz point, Phys. Rev. D 79 (2009) 084008 [arXiv:0901.3775] [INSPIRE].

[121] R.L. Arnowitt, S. Deser and C.W. Misner, The Dynamics of general relativity, Gen. Rel. Grav. 40 (2008) 1997 [gr-qc/0405109] [INSPIRE].

[122] S. Mukohyama, Hor̆ava-Lifshitz cosmology: a review, Class. Quant. Grav. 27 (2010) 223101 [arXiv: 1007.5199] [INSPIRE].

[123] T.P. Sotiriou, Hořava-Lifshitz gravity: a status report, J. Phys. Conf. Ser. 283 (2011) 012034 [arXiv: 1010.3218] [INSPIRE].

[124] D. Blas, O. Pujolàs and S. Sibiryakov, Consistent extension of Hor̆ava gravity, Phys. Rev. Lett. 104 (2010) 181302 [arXiv:0909.3525] [INSPIRE].

[125] D. Blas, O. Pujolàs and S. Sibiryakov, Models of non-relativistic quantum gravity: the good, the bad and the healthy, JHEP 04 (2011) 018 [arXiv: 1007.3503] [INSPIRE].

[126] T. Jacobson, Extended Hor̆ava gravity and Einstein-aether theory, Phys. Rev. D 81 (2010) 101502 [Erratum ibid. D 82 (2010) 129901] [arXiv: 1001.4823] [INSPIRE].

[127] T. Jacobson, Undoing the twist: the Hořava limit of Einstein-aether theory, Phys. Rev. D 89 (2014) 081501 [arXiv: 1310.5115] [INSPIRE].

[128] T. Jacobson and D. Mattingly, Gravity with a dynamical preferred frame, Phys. Rev. D 64 (2001) 024028 [gr-qc/0007031] [inSPIRE].

[129] C. Eling and T. Jacobson, Static postNewtonian equivalence of GR and gravity with a dynamical preferred frame, Phys. Rev. D 69 (2004) 064005 [gr-qc/0310044] [INSPIRE].

[130] T. Jacobson, Einstein-aether gravity: a status report, PoS(QG-PH) 020 [arXiv:0801.1547] [INSPIRE].

[131] E. Kiritsis and G. Kofinas, Hořava-Lifshitz Cosmology, Nucl. Phys. B 821 (2009) 467 [arXiv:0904.1334] [INSPIRE].

[132] P. Hořava and C.M. Melby-Thompson, General covariance in quantum gravity at a Lifshitz point, Phys. Rev. D 82 (2010) 064027 [arXiv:1007.2410] [INSPIRE].

[133] A.M. da Silva, An alternative approach for general covariant Hořava-Lifshitz gravity and matter coupling, Class. Quant. Grav. 28 (2011) 055011 [arXiv: 1009.4885] [INSPIRE]. 
[134] T. Griffin, P. Hořava and C.M. Melby-Thompson, Lifshitz gravity for Lifshitz holography, Phys. Rev. Lett. 110 (2013) 081602 [arXiv:1211.4872] [InSPIRE].

[135] M. Alishahiha and H. Yavartanoo, Conformally Lifshitz solutions from Hor̆avaLifshitz Gravity, Class. Quant. Grav. 31 (2014) 095008 [arXiv: 1212.4190] [INSPIRE].

[136] S. Janiszewski, Asymptotically hyperbolic black holes in Hor̆ava gravity, arXiv:1401.1463 [INSPIRE].

[137] S. Janiszewski, A. Karch, B. Robinson and D. Sommer, Charged black holes in Hořava gravity, JHEP 04 (2014) 163 [arXiv: 1401.6479] [INSPIRE].

[138] T.-J. Li, Y.-H. Qi, Y.-L. Wu and Y.-L. Zhang, Topolgical charged black holes in generalized Hořava-Lifshitz gravity, Phys. Rev. D 90 (2014) 124070 [arXiv:1405.4457] [INSPIRE].

[139] O. Saremi and D.T. Son, Hall viscosity from gauge/gravity duality, JHEP 04 (2012) 091 [arXiv: 1103.4851] [INSPIRE].

[140] J.-W. Chen, N.-E. Lee, D. Maity and W.-Y. Wen, A holographic model for Hall viscosity, Phys. Lett. B 713 (2012) 47 [arXiv:1110.0793] [INSPIRE].

[141] J.-W. Chen, S.-H. Dai, N.-E. Lee and D. Maity, Novel parity violating transport coefficients in 2+1 dimensions from holography, JHEP 09 (2012) 096 [arXiv: 1206.0850] [INSPIRE].

[142] H. Liu, H. Ooguri, B. Stoica and N. Yunes, Spontaneous generation of angular momentum in holographic theories, Phys. Rev. Lett. 110 (2013) 211601 [arXiv:1212.3666] [INSPIRE].

[143] D.-C. Zou and B. Wang, Holographic parity violating charged fluid dual to Chern-Simons modified gravity, Phys. Rev. D 89 (2014) 064036 [arXiv:1306.5486] [INSPIRE].

[144] D.T. Son and C. Wu, Holographic spontaneous parity breaking and emergent Hall viscosity and angular momentum, JHEP 07 (2014) 076 [arXiv: 1311.4882] [INSPIRE].

[145] H. Liu, H. Ooguri and B. Stoica, Angular momentum generation by parity violation, Phys. Rev. D 89 (2014) 106007 [arXiv:1311.5879] [InSPIRE].

[146] C. Wu, Angular momentum generation from holographic Chern-Simons models, JHEP 12 (2014) 090 [arXiv: 1311.6368] [INSPIRE].

[147] H. Liu, H. Ooguri and B. Stoica, Hall viscosity and angular momentum in gapless holographic models, Phys. Rev. D 90 (2014) 086007 [arXiv:1403.6047] [INSPIRE].

[148] R.G. Leigh, A.C. Petkou and P.M. Petropoulos, Holographic fluids with vorticity and analogue gravity, JHEP 11 (2012) 121 [arXiv:1205.6140] [INSPIRE].

[149] R. Alicki, Universal correction to the quantum Hall current in a nonuniform magnetic field, Phys. Rev. Lett. 72 (1994) 2620.

[150] C. Kittel, Quantum Theory of Solids, Wiley, New York U.S.A. (1963).

[151] G.Y. Cho, Y. You and E. Fradkin, Geometry of fractional quantum Hall fluids, Phys. Rev. B 90 (2014) 115139 [arXiv:1406.2700] [INSPIRE].

[152] P. Nikolic and S. Sachdev, Renormalization-group fixed points, universal phase diagram and $1 / N$ expansion for quantum liquids with interactions near the unitarity limit, Phys. Rev. A 75 (2007) 033608 [cond-mat/0609106] [INSPIRE].

[153] T. Griffin, P. Hořava and C.M. Melby-Thompson, Conformal Lifshitz gravity from holography, JHEP 05 (2012) 010 [arXiv:1112.5660] [INSPIRE]. 
[154] P. Hořava and C.M. Melby-Thompson, Anisotropic conformal infinity, Gen. Rel. Grav. 43 (2011) 1391 [arXiv: 0909.3841] [INSPIRE].

[155] S.F. Ross and O. Saremi, Holographic stress tensor for non-relativistic theories, JHEP 09 (2009) 009 [arXiv: 0907.1846] [INSPIRE].

[156] S.F. Ross, Holography for asymptotically locally Lifshitz spacetimes, Class. Quant. Grav. 28 (2011) 215019 [arXiv:1107.4451] [INSPIRE].

[157] F. Wilczek, Two applications of axion electrodynamics, Phys. Rev. Lett. 58 (1987) 1799 [INSPIRE].

[158] S.M. Carroll, G.B. Field and R. Jackiw, Limits on a Lorentz and parity violating modification of electrodynamics, Phys. Rev. D 41 (1990) 1231 [INSPIRE].

[159] T. Can, M. Laskin and P. Wiegmann, Fractional quantum Hall effect in a curved space: gravitational anomaly and electromagnetic response, Phys. Rev. Lett. 113 (2014) 046803 [arXiv: 1402.1531] [INSPIRE].

[160] R. Jackiw and S.Y. Pi, Chern-Simons modification of general relativity, Phys. Rev. D 68 (2003) 104012 [gr-qc/0308071] [INSPIRE].

[161] D. Grumiller, R.B. Mann and R. McNees, Dirichlet boundary value problem for Chern-Simons modified gravity, Phys. Rev. D 78 (2008) 081502 [arXiv:0803.1485] [INSPIRE].

[162] C.L. Kane and M.P.A. Fisher, Quantized thermal transport in the fractional quantum Hall effect, Phys. Rev. B 55 (1997) 15832 [cond-mat/9603118].

[163] M. Geracie and D.T. Son, Thermal transport in a noncommutative hydrodynamics, arXiv: 1407.4460 [INSPIRE].

[164] N. Read and D. Green, Paired states of fermions in two-dimensions with breaking of parity and time reversal symmetries and the fractional quantum Hall effect, Phys. Rev. B 61 (2000) 10267 [cond-mat/9906453] [INSPIRE].

[165] A. Cappelli, M. Huerta and G.R. Zemba, Thermal transport in chiral conformal theories and hierarchical quantum Hall states, Nucl. Phys. B 636 (2002) 568 [cond-mat/0111437] [INSPIRE].

[166] S. Ryu, J.E. Moore and A.W.W. Ludwig, Electromagnetic and gravitational responses and anomalies in topological insulators and superconductors, Phys. Rev. B 85 (2012) 045104 [arXiv: 1010.0936$]$ [INSPIRE].

[167] Z. Wang, X.-L. Qi and S.-C. Zhang, Topological field theory and thermal responses of interacting topological superconductors, Phys. Rev. B 84 (2011) 014527 [arXiv:1011.0586] [INSPIRE].

[168] M. Stone, Gravitational anomalies and thermal Hall effect in topological insulators, Phys. Rev. B 85 (2012) 184503 [arXiv:1201.4095] [INSPIRE].

[169] G.W. Moore and N. Read, Nonabelions in the fractional quantum Hall effect, Nucl. Phys. B 360 (1991) 362 [INSPIRE].

[170] F.D.M. Haldane, Fractional quantization of the Hall effect: a hierarchy of incompressible quantum fluid states, Phys. Rev. Lett. 51 (1983) 605 [InSPIRE].

[171] K. Jensen, M. Kaminski, P. Kovtun, R. Meyer, A. Ritz et al., Parity-violating hydrodynamics in 2+1 dimensions, JHEP 05 (2012) 102 [arXiv:1112.4498] [INSPIRE]. 
[172] R.-G. Cai, T.-J. Li, Y.-H. Qi and Y.-L. Zhang, Incompressible Navier-Stokes equations from Einstein gravity with Chern-Simons term, Phys. Rev. D 86 (2012) 086008 [arXiv:1208.0658] [INSPIRE].

[173] F.M. Haehl and M. Rangamani, Comments on Hall transport from effective actions, JHEP 10 (2013) 074 [arXiv: 1305.6968] [INSPIRE].

[174] M. Kaminski and S. Moroz, Non-relativistic parity-violating hydrodynamics in two spatial dimensions, Phys. Rev. B 89 (2014) 115418 [arXiv:1310.8305] [INSPIRE].

[175] A. Lucas and P. Surówka, Phenomenology of nonrelativistic parity-violating hydrodynamics in 2+1 dimensions, Phys. Rev. E 90 (2014) 063005 [arXiv:1403.5239] [INSPIRE].

[176] N.R. Cooper, B.I. Halperin and I.M. Ruzin, Thermoelectric response of an interacting two-dimensional electron gas in quantizing magnetic field, Phys. Rev. B 55 (1997) 2344 [cond-mat/9607001].

[177] M. Stone, Superfluid dynamics of the fractional quantum Hall state, Phys. Rev. B 42 (1990) 212.

[178] S.A. Hartnoll and P. Kovtun, Hall conductivity from dyonic black holes, Phys. Rev. D 76 (2007) 066001 [arXiv:0704.1160] [inSPIRE].

[179] M. Stone and R. Roy, Edge modes, edge currents, and gauge invariance in superfluids and superconductors, Phys. Rev. B 69 (2004) 184511 [cond-mat/0308034].

[180] Y. Tsutsumi and K. Machida, Edge mass current and the role of Majorana fermions in A-phase superfluid ${ }^{3}$ He, Phys. Rev. B 85 (2012) 100506 [arXiv:1110.5409].

[181] J.A. Sauls, Surface states, edge currents and the angular momentum of chiral p-wave superfluids, Phys. Rev. B 84 (2011) 214509 [arXiv: 1209.5501].

[182] J.K. Jain, Composite fermion approach for the fractional quantum Hall effect, Phys. Rev. Lett. 63 (1989) 199 [INSPIRE].

[183] B. Yang, Geometric aspects and neutral excitations in the fractional quantum Hall effect, arXiv:1312.2630 [INSPIRE].

[184] L. Susskind, The quantum Hall fluid and noncommutative Chern-Simons theory, hep-th/0101029 [INSPIRE].

[185] E. Fradkin, V. Jejjala and R.G. Leigh, Noncommutative Chern-Simons for the quantum Hall system and duality, Nucl. Phys. B 642 (2002) 483 [cond-mat/0205653] [INSPIRE].

[186] J. Bellorín and A. Restuccia, On the consistency of the Horava theory, Int. J. Mod. Phys. D 21 (2012) 1250029 [arXiv: 1004.0055] [inSPIRE].

[187] J. Kluson, Note about Hamiltonian formalism of healthy extended Hor̆ava-Lifshitz gravity, JHEP 07 (2010) 038 [arXiv: 1004.3428] [INSPIRE].

[188] J. Bellorín and A. Restuccia, Consistency of the Hamiltonian formulation of the lowest-order effective action of the complete Hor̆ava theory, Phys. Rev. D 84 (2011) 104037 [arXiv:1106.5766] [INSPIRE].

[189] J. Bellorín, A. Restuccia and A. Sotomayor, Non-perturbative analysis of the constraints and the positivity of the energy of the complete Horava theory, Phys. Rev. D 85 (2012) 124060 [arXiv: 1205.2284] [INSPIRE]. 